\title{
Three-point functions in ABJM and Bethe Ansatz
}

\author{
Peihe Yang, ${ }^{a}$ Yunfeng Jiang, ${ }^{b, c, d}$ Shota Komatsu ${ }^{d, e}$ and Jun-Bao $\mathbf{W} \mathbf{u}^{a, f, g, 1}$ \\ ${ }^{a}$ Center for Joint Quantum Studies and Department of Physics, \\ School of Science, Tianjin University, \\ 135 Yaguan Road, Tianjin 300350, P.R. China \\ ${ }^{b}$ School of physics, Southeast University, \\ Nanjing 211189, P.R. China \\ ${ }^{c}$ Shing-Tung Yau Center of Southeast University, \\ Nanjing 210096, P.R. China \\ ${ }^{d}$ Department of Theoretical Physics, CERN, \\ 1 Esplanade des Particules, 1211 Meyrin, Switzerland \\ e School of Natural Sciences, Institute for Advanced Study, \\ 1 Einstein Dr. Princeton, NJ 08540, U.S.A. \\ ${ }^{f}$ Peng Huangwu Center for Fundamental Theory, \\ Hefei, Anhui 230026, P.R. China \\ ${ }^{g}$ Center for High Energy Physics, Peking University, \\ 5 Yiheyuan Rd, Beijing 100871, P.R. China \\ E-mail: peihe_yang@tju.edu.cn, Yunfeng.Jiang@cern.ch, \\ shota.komatsu@cern.ch, junbao.wu@tju.edu.cn
}

ABSTRACT: We develop an integrability-based framework to compute structure constants of two sub-determinant operators and a single-trace non-BPS operator in ABJM theory in the planar limit. In this first paper, we study them at weak coupling using a relation to an integrable spin chain. We first develop a nested Bethe ansatz for an alternating SU(4) spin chain that describes single-trace operators made out of scalar fields. We then apply it to the computation of the structure constants and show that they are given by overlaps between a Bethe eigenstate and a matrix product state. We conjecture that the determinant operator corresponds to an integrable matrix product state and present a closed-form expression for the overlap, which resembles the so-called Gaudin determinant. We also provide evidence for the integrability of general sub-determinant operators. The techniques developed in this paper can be applied to other quantities in ABJM theory including three-point functions of single-trace operators.

Keywords: 1/ $N$ Expansion, AdS-CFT Correspondence, Bethe Ansatz, Integrable Field Theories

ARXIV EPRINT: 2103.15840

\footnotetext{
${ }^{1}$ Corresponding author
} 


\section{Contents}

1 Introduction 1

2 Setup and Kinematics 4

2.1 Generalities 4

2.2 Structures of two- and three-point functions 6

2.3 Twisted translation 9

3 SU(4) invariant alternating spin chain $\quad 12$

3.1 Hamiltonian and Bethe equations 12

$\begin{array}{ll}3.2 \text { Coordinate Bethe ansatz } & 14\end{array}$

4 Matrix product states from giant gravitons $\quad 18$

$\begin{array}{lll}4.1 & \text { Derivation of matrix product states } & 18\end{array}$

4.2 Two-point function: saddle point and MPS 22

4.3 Structure constants of BPS single-trace operators 24

4.4 Structure constants of non-BPS single-trace operators 25

5 Main results $\quad 27$

5.1 Summary of results 27

5.2 Norms and Gaudin determinant 29

5.3 Some details on the overlap 31

6 Conclusion $\quad 32$

A Partially contracted giant graviton 33

B Derivation of BPS three-point funcitons 35

C Data and numerics $\quad 36$

C.1 Numerical solutions to the Bethe equations 36

C.2 Overlaps for non-maximal giant gravitons 40

$\begin{array}{ll}\text { D Nested algebraic Bethe ansatz } & 40\end{array}$

\section{Introduction}

Over the last fifteen years, there have been breathtaking developments in solving $\mathcal{N}=4$ supersymmetric Yang-Mills theory (SYM) in four dimensions in the large $N_{c}$ limit. This was mainly thanks to the successful application of integrability, a method originally invented to 
solve special two-dimensional systems. A key finding which triggered this burst of activity was made by Minahan and Zarembo [1], who recognized that the action of the dilatation operator on a subclass of operators, called single-trace operators, can be mapped to a Hamiltonian of an integrable spin chain. Subsequently the idea was generalized and refined in various directions, and by now the integrability was applied not just to the spectrum of single-trace operators [2-6] but also to their correlation functions [7-23] and even to their $1 / N_{c}$ corrections $^{1}[25-28]$. All these progresses indicate that we are close to a complete solution to an interacting gauge theory in four dimensions; a feat never achieved in the long history of theoretical physics.

More recently the integrability approach was applied to quantities that are far beyond single-trace operators: the paper $[29,30]$ showed that the correlation function of two determinant operators and one single-trace operator can be computed exactly using the integrability machinery. This came as a surprise since the quantum numbers of determinant operators scale with $N_{c}$, and even performing the perturbative computation is a nontrivial task. Nevertheless, by a judicious rewriting one can map these observables to overlaps of states in an integrable two-dimensional system (a spin chain at weak coupling and a string worldsheet at strong coupling), and compute them exactly [29, 30].

In this series of papers $[31,32]$, we generalize the analysis of $[29,30]$ to $\mathcal{N}=6$ supersymmetric Chern-Simons-matter theory in three dimensions, constructed by Aharony, Bergman, Jafferis and Maldacena [33]; ABJM theory for short. Soon after the construction of ABJM theory, it was realized in [34-37] that ABJM theory is also integrable in the large $N_{c}$ limit as far as the spectrum of single-trace operators ${ }^{2}$ is concerned. However, unlike $\mathcal{N}=4$ SYM, not much is known beyond the spectrum. In particular, we are still lacking an integrability-based framework to compute correlation functions at finite 't Hooft coupling. The goal of this series of papers is to propose and establish the first of such frameworks. In this first paper, we focus on the computation at weak coupling and show that the treelevel structure constants of two determinant operators and one single-trace operator can be computed by overlaps between a matrix product state and a Bethe eigenstate in an integrable spin chain. Furthermore, we present evidence that the relevant matrix product state preserves integrability and conjecture a closed-form expression for the overlaps in terms of Gaudin-like determinants. Similar expressions were found in various different contexts, ranging from the study of quench dynamics [40-48] to the defect one-point functions in $\mathcal{N}=4$ SYM $[49-58]$.

We should also mention an important difference from $\mathcal{N}=4 \mathrm{SYM}$. In $\mathcal{N}=4 \mathrm{SYM}$, the result in [59] suggests that only the determinant operator corresponds to an integrable boundary state and all the sub-determinant operators are not integrable ${ }^{3}$. By contrast,

\footnotetext{
${ }^{1}$ See also recent interesting work on non-planar anomalous dimensions [24].

${ }^{2}$ The full solution to the spectral problem was obtained by Quantum Spectral Curve [38, 39].

${ }^{3}$ We should however note that the (non-)integrability of the sub-determinant operators is not fully settled even for $\mathcal{N}=4 \mathrm{SYM}$. For instance, there are results on the spectrum of open string attached to the subdeterminant operators which suggest the sub-determinant operators might actually correspond to integrable boundary states $[60,61]$. It would be interesting to revisit this question in view of recent discovery of a new integrable boundary condition in $A d S_{5} \times S^{5}$ [62], which was missed in the classification in [63].
} 
the analysis of this paper suggests that the sub-determinant operators are also integrable in ABJM theory, at least at tree level. The possibility of having such a family of integrable boundary states motivates further study of these operators in ABJM theory.

Before describing the contents of the paper, let us explain a couple of more motivations. Two rather obvious reasons were already mentioned; 1 . we can test the formalism developed in $[29,30]$ in other theories, and 2. we establish the first integrability-based framework to compute correlation functions in ABJM theory. Yet another motivation comes from the fact that the structure constants in ABJM theory receive quantum corrections even when all the operators are BPS [64-66]. For the purpose of checking the AdS/CFT correspondence, this is more like a curse than a blessing since it inhibits a direct comparison between the results at weak coupling and the results in supergravity. On the other hand, this feature makes ABJM theory an ideal testing ground for the integrability approach to the correlation functions: in the integrability description, the BPS operator corresponds to the vacuum state of the spin chain and provides the simplest setup for the computation. Unfortunately, this setup was "too simple" for $\mathcal{N}=4$ SYM since the quantum corrections all vanish [67]. By contrast, in ABJM theory the setup provides a simple yet nontrivial test of the formalism because of the dependence on the coupling constant. In addition, structure constants of BPS single-trace operators in planar ABJM theory can be computed by supersymmetric localization [68-76]. At present, localization computation has not been generalized to sub-determinant operators, but if we succeed in doing so, we will be able to compare two rather different approaches and deepen our understanding on the relation between them.

The rest of the paper is organized as follows. In section 2, we explain the setup to be discussed in this paper: in ABJM theory, even the three-point functions of BPS operators can have several $R$-symmetry tensor structures and therefore depend on a multitude of structure constants. In order to simplify the analysis, we focus on the twisted-translated frame. We show that the structure constant computed in the twisted-translated frame is a particular linear combination of the full structure constants. ${ }^{4}$ In section 3 , we first review the basic properties of the SU(4) invariant alternating spin chain, such as the Hamiltonian, the Bethe equations and the relation to the single-trace operator. We then develop the nested coordinate Bethe ansatz and write down wave functions of Bethe states. In section 4 , we derive a matrix product state representation of the structure constant of two giant gravitons and a single-trace BPS operator. Such analyses were performed in $\mathcal{N}=4 \mathrm{SYM}$ in $[29,30,59]$ and in $\mathrm{ABJ}(\mathrm{M})$ theory in [77]. The novelty of our analysis is that we derive an explicit expression for the matrix product state for the sub-determinant operators in the twisted-translated frame which can be readily used to evaluate the structure constants. We also evaluate the tree-level structure constants of two non-maximal sub-determinant operators and a single-trace BPS operator. In section 5, we compute the structure constants using the results in the preceding sections and present our conjecture. As was the case with $\mathcal{N}=4 \mathrm{SYM}$, the result for determinant operators exhibits a nontrivial

\footnotetext{
${ }^{4}$ In principle, we can recover the full structure constants by acting the $R$-symmetry transformation to each operator, which in the spin-chain language corresponds to adding zero-momentum magnons.
} 
selection rule on the rapidities and is given by a ratio of Gaudin-like determinants. These imply that the determinant operators in ABJM theory correspond to integrable boundary states. One notable difference from $\mathcal{N}=4 \mathrm{SYM}$ is that these features persist also for sub-determinant operators, indicating that ABJM theory admits a family of integrable boundary states. Our results also provide the first example of integrable matrix product states in the alternating spin chain. Finally in section 6 , we conclude and discuss future directions. Several appendices are included to explain technical details.

\section{Setup and Kinematics}

\subsection{Generalities}

Basics. ABJM theory is a $\mathcal{N}=6$ superconformal Chern-Simons matter theory in three dimensions with a product gauge group $\mathrm{U}(N)_{k} \times \mathrm{U}(N)_{-k}$ where $k$ is the Chern-Simons level. It consists of two sets of gauge fields and matter fields in the bi-fundamental representations. See $[33,78]$ for the explicit form of the Lagrangian. See also [79] for a review of the integrability properties of ABJM theory.

A distinguishing feature of ABJM theory (as compared to $\mathcal{N}=4$ SYM) is that it admits two different large $N$ limits: the first limit is called the $M$-theory limit and can be defined by $N \rightarrow \infty$ with $k$ fixed. This limit has attracted much attention since it is dual to a M-theory on $A d S_{4} \times S^{7} / Z_{k}$ and provides one of the most concrete non-perturbative definitions of the M-theory currently available. Unfortunately this limit is difficult to study on the field theory side since it corresponds to a strong coupling limit (unless we focus on the BPS observables and use supersymmetric localization). We therefore study the second limit in this paper, namely the planar limit. The planar limit is defined by sending $N \rightarrow \infty$ while keeping the 't Hooft coupling

$$
\lambda \equiv \frac{N}{k},
$$

fixed. As is the case with the standard planar limit of Yang-Mills theories, the observables in the planar limit can be computed by the planar diagrams - diagrams that can be drawn on a genus 0 Riemann surface. The holographic dual of this limit is given by type-IIA superstring theory in $A d S_{4} \times C P^{3}$.

The main subject of this paper involves gauge invariant local operators in ABJM made out of scalar fields. ABJM theory has two sets of scalar fields; the one that transforms as $(\square, \bar{\square} ; \mathbf{4})$ and the other that transforms as $(\bar{\square}, \square ; \overline{\mathbf{4}})$ under the $\mathrm{U}(N)_{k} \times \mathrm{U}(N)_{-k}$ gauge groups and $\mathrm{SU}(4) R$-symmetry,

$$
\begin{array}{cll}
Y^{I}: & (\square, \bar{\square} ; \mathbf{4}), & \Delta^{0}=1 / 2, \\
\bar{Y}_{I}: & (\bar{\square}, \square ; \overline{4}), & \Delta^{0}=1 / 2,
\end{array}
$$

where $I=1, \ldots, 4$ and $\Delta^{0}$ is the mass dimension. The simplest gauge invariant operators constructed out of such fields are single-trace operators, which in general take the following form:

$$
\operatorname{tr}\left(Y^{I_{1}} \bar{Y}_{J_{1}} \cdots Y^{I_{L}} \bar{Y}_{J_{L}}\right)+\cdots
$$


As is clear from this expression, it consists of an alternating sequence of $Y^{I}$ fields and $\bar{Y}_{I}$ fields. Because of this feature, the spin chain that describes the anomalous dimension of such an operator is an alternating spin chain, in which spins living on odd sites are distinct from spins living on even sites. We will give a more detailed description of the spin chain and its Bethe ansatz in section 3.

BPS operators and giant gravitons. General single-trace operators (2.3) do not preserve any supersymmetry. However, for a special choice of the $R$-symmetry indices, they become BPS and invariant under $1 / 3$ of the supersymmetry transformations. Written explicitly they take the following form,

$$
\mathcal{O}_{L}^{\circ}(x ; n, \bar{n}) \equiv \operatorname{tr}\left[((n \cdot Y)(\bar{n} \cdot \bar{Y}))^{L}\right],
$$

where the supercript $\circ$ is to indicate that the operator is $\mathrm{BPS}^{5}$ and we have

$$
n \cdot Y \equiv \sum_{I=1}^{4} n_{I} Y^{I}, \quad \bar{n} \cdot \bar{Y} \equiv \sum_{I=1}^{4} \bar{n}^{I} \bar{Y}_{I} .
$$

Here $n$ and $\bar{n}$ are four-component vectors specifying the $R$-symmetry polarizations of the operator and they need to satisfy

$$
n \cdot \bar{n}=0
$$

in order for the operator to be BPS.

Another class of BPS operators considered in this paper are giant gravitons [81-84]. They are defined in terms of sub-determinants as

$$
\mathcal{D}_{M}(x ; n, \bar{n}) \equiv \frac{1}{M !} \delta_{\left[a_{1} \cdots a_{M}\right]}^{\left[b_{1} \cdots b_{M}\right]}[(n \cdot Y)(\bar{n} \cdot \bar{Y})]_{b_{1}}^{a_{1}} \cdots[(n \cdot Y)(\bar{n} \cdot \bar{Y})]_{b_{M}}^{a_{M}},
$$

with $n \cdot \bar{n}=0$ and

$$
\delta_{\left[a_{1} \cdots a_{M}\right]}^{\left[b_{1} \cdots b_{M}\right]} \equiv \sum_{\sigma \in S_{M}}(-1)^{|\sigma|} \delta_{a_{\sigma_{1}}}^{b_{1}} \cdots \delta_{b_{\sigma_{M}}}^{b_{M}}
$$

The operator with a maximal $R$-charge $(M=N)$ is called the maximal giant gravitons while others $(M<N)$ are called non-maximal giant gravitons.

Alternatively, they can be defined in terms of the antisymmetric Schur polynomial [81, $82,85,86]$ as follows:

$$
\mathcal{D}_{M}(x ; n, \bar{n})=\frac{1}{M !} \sum_{\sigma \in S_{M}} \chi_{\mathrm{A}_{M}}(\sigma)[(n \cdot Y)(\bar{n} \cdot \bar{Y})]_{a_{1}}^{a_{\sigma_{1}}} \cdots[(n \cdot Y)(\bar{n} \cdot \bar{Y})]_{a_{M}}^{a_{\sigma_{M}}}
$$

where $\chi_{\mathrm{A}_{M}}$ is a Schur polynomial for the totally antisymmetric representation of size $M$. See e.g. [77] for explicit definitions. To study the correlation functions of giant gravitons,

\footnotetext{
${ }^{5}$ Here we are following the notation used in [80].
} 
it is often convenient to consider a generating function $\mathcal{G}(x ; n, \bar{n}, t)$ defined by ${ }^{6}$

$$
\mathcal{G}(x ; n, \bar{n}, t) \equiv \operatorname{det}\left(\begin{array}{cc}
\mathbf{1} & -t(\bar{n} \cdot \bar{Y}) \\
t(n \cdot Y) & \mathbf{1}
\end{array}\right)=\operatorname{det}\left[\mathbf{1}+t^{2}(n \cdot Y)(\bar{n} \cdot \bar{Y})\right] .
$$

To extract the giant gravitons with a fixed charges, we perform the integral of $t$;

$$
\mathcal{D}_{M}(x ; n, \bar{n})=\oint \frac{\mathrm{d} t}{2 \pi i t^{1+2 M}} \mathcal{G}(x ; n, \bar{n}, t) .
$$

Holographic dual. Let us also briefly review the dual description of giant gravitons although it is not directly relevant for the analysis performed in this paper. There are two classes of giant gravitons in $A d S_{4} \times C P^{3}$ known in the literature which are conjectured to be dual to $1 / 3$ BPS operators. ${ }^{7}$ The first class is the D2 branes extended in $S^{2}$ inside $A d S_{4}$ [87-89]. These branes are known to be dual to symmetric Schur polynomials and are analogs of the dual giant gravitons in $\mathcal{N}=4 \mathrm{SYM}$. The other class is the D4 branes which are point-like in $A d S_{4}$ and extended in the $C P^{3}$ direction [83, 87, 90-94]. They are dual to antisymmetric Schur polynomials and are the subject of this paper. In the upcoming paper [31], we will study the correlation functions from these holographic perspectives.

\subsection{Structures of two- and three-point functions}

We now summarize the structures of two- and three-point functions and the constraints from symmetry, emphasizing the differences from $\mathcal{N}=4 \mathrm{SYM}$.

BPS two-point functions. Let us first consider the two-point functions of $1 / 3$ BPS single-trace operators, $\left\langle\mathcal{O}_{L_{1}}^{\circ} \mathcal{O}_{L_{2}}^{\circ}\right\rangle$. Since $n_{k}$ 's transform as the anti-fundamentals of SU(4) while $\bar{n}_{k}$ 's transform as the fundamentals of $\mathrm{SU}(4)$, the $\mathrm{SU}(4)$ symmetry determines the structure of the two-point function to be

$$
\left\langle\mathcal{O}_{L_{1}}^{\circ} \mathcal{O}_{L_{2}}^{\circ}\right\rangle=\delta_{L_{1}, L_{2}} \mathcal{N}_{\mathcal{O}_{L_{1}}^{\circ}}\left(d_{12} d_{21}\right)^{L_{1}}
$$

where $\mathcal{N}_{\mathcal{O}_{L}^{\circ}}$ is the normalization constant, which at weak coupling reads

$$
\mathcal{N}_{\mathcal{O}_{L}^{\circ}}=L \lambda^{2 L}
$$

while $d_{i j}$ 's are defined by

$$
d_{i j} \equiv \frac{n_{i} \cdot \bar{n}_{j}}{\left|x_{i j}\right|} \quad\left|x_{i j}\right| \equiv\left|x_{i}-x_{j}\right| .
$$

\footnotetext{
${ }^{6}$ The second equality in (2.10) follows from the invariance of the determinant under the addition and the subtraction of rows or columns,

$$
\operatorname{det}\left(\begin{array}{cc}
\mathbf{1} & -t(\bar{n} \cdot \bar{Y}) \\
t(n \cdot Y) & \mathbf{1}
\end{array}\right)=\operatorname{det}\left(\begin{array}{cc}
\mathbf{1} & \mathbf{0} \\
t(n \cdot Y) & \mathbf{1}+t^{2}(n \cdot Y)(\bar{n} \cdot \bar{Y})
\end{array}\right)=\operatorname{det}\left[\mathbf{1}+t^{2}(n \cdot Y)(\bar{n} \cdot \bar{Y})\right] .
$$

${ }^{7}$ Some giant gravitions with more or less supersymmetries were also studied in the papers listed below in this paragraph.
} 
Note that, unlike $\mathcal{N}=4 \mathrm{SYM}, d_{i j}$ here is not symmetric under the exchange of indices: $d_{i j} \neq d_{j i}$.

The symmetry is powerful enough to determine the two-point functions of (sub)determinant operators as well:

$$
\left\langle\mathcal{D}_{M}\left(x_{1}, n_{1}, \bar{n}_{1}\right) \mathcal{D}_{M}\left(x_{2}, n_{2}, \bar{n}_{2}\right)\right\rangle=\mathcal{N}_{\mathcal{D}_{M}}\left(d_{12} d_{21}\right)^{M} .
$$

Here again $\mathcal{N}_{\mathcal{D}_{M}}$ is the normalization constant.

BPS three-point functions. We then discuss the three-point functions of $1 / 3$-BPS operators. This is where ABJM theory shows significant differences from $\mathcal{N}=4 \mathrm{SYM}$ :

- In $\mathcal{N}=4 \mathrm{SYM}$, the $R$-symmetry structure of the BPS three-point functions is determined completely by the symmetry. On the other hand, the BPS three-point function in ABJM theory admits several different structures.

- The structure constant of $1 / 2$-BPS operators in $\mathcal{N}=4$ SYM does not depend on the 't Hooft coupling. By contrast, the structure constant of $1 / 3$-BPS operators in ABJM theory is a nontrivial function of the 't Hooft coupling.

To see this explicitly, let us consider the three-point function of BPS single-trace operators. Imposing the SU(4) symmetry, one finds that the three-point function has a multitude of allowed structures labelled by $p$ which is either an integer or a half-integer ${ }^{8}$ [95],

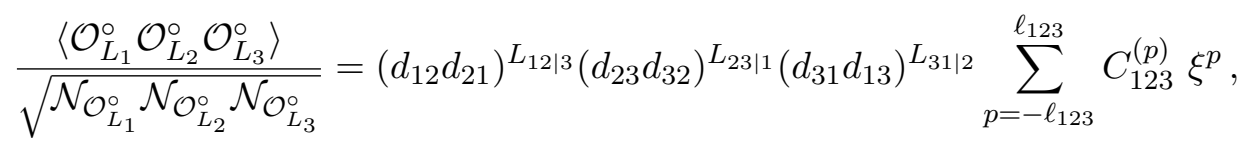

with

$$
L_{i j \mid k} \equiv \frac{L_{i}+L_{j}-L_{k}}{2}, \quad \ell_{123} \equiv \min \left[L_{12 \mid 3}, L_{23 \mid 1}, L_{31 \mid 2}\right] .
$$

Here $\xi$ is the $\mathrm{SU}(4)$ cross ratio

$$
\xi \equiv \frac{d_{12} d_{23} d_{31}}{d_{21} d_{32} d_{13}}=\frac{\left(n_{1} \cdot \bar{n}_{2}\right)\left(n_{2} \cdot \bar{n}_{3}\right)\left(n_{3} \cdot \bar{n}_{1}\right)}{\left(n_{2} \cdot \bar{n}_{1}\right)\left(n_{3} \cdot \bar{n}_{2}\right)\left(n_{1} \cdot \bar{n}_{3}\right)},
$$

and $C_{123}^{(p)}$ 's are a set of structure constants. These structure constants can be readily computed at tree level by Wick contractions [64, 84]:

$$
C_{123}^{(p)} \stackrel{\lambda \rightarrow 0}{=} \frac{\sqrt{L_{1} L_{2} L_{3}}}{N} \times\left\{\begin{array}{ll}
2 \delta_{p, 0} & \sum_{k=1}^{3} L_{k}: \text { even } \\
\delta_{p, \frac{1}{2}}+\delta_{p,-\frac{1}{2}} & \sum_{k=1}^{3} L_{k}: \text { odd }
\end{array} .\right.
$$

On the other hand, $[84,96]$ performed the computation at strong coupling and the result reads

$$
C_{123}^{(p)} \stackrel{\lambda \rightarrow \infty}{=} \frac{1}{N} \sqrt{\frac{h(\lambda)}{\pi}} \frac{\prod_{j=1}^{3} \sqrt{1+2 L_{j}} \Gamma\left[1+L_{j}\right]}{\Gamma\left[1+\frac{L_{1}+L_{2}+L_{3}}{2}\right]} \prod_{\{i, j, k\}} \frac{\Gamma\left[1+\frac{L_{i j \mid k}}{2}\right]}{\Gamma\left[1-p+\frac{L_{i j \mid k}}{2}\right] \Gamma\left[1+p+\frac{L_{i j \mid k}}{2}\right]},
$$

\footnotetext{
${ }^{8} p$ takes an integer-value when $\sum_{k} L_{k}$ is even while it takes a half-integer value when $\sum_{k} L_{k}$ is odd.
} 
where $\prod_{\{i, j, k\}}$ denotes a product over cyclic permutations of $\{1,2,3\}$ and $h(\lambda)$ is the socalled interpolating function [97] which can be expanded at weak and strong couplings as

$$
h(\lambda)= \begin{cases}\lambda-\frac{\pi^{2} \lambda^{3}}{3}+\cdots & \lambda \rightarrow 0 \\ \sqrt{\frac{1}{2}\left(\lambda-\frac{1}{24}\right)}-\frac{\log 2}{\pi}+\cdots & \lambda \rightarrow \infty .\end{cases}
$$

The difference between (2.19) and (2.20) provides clear evidence for the coupling dependence of structure constants $C_{123}^{(p)}$.

The expression (2.16) can be readily generalized to the three-point function of two sub-determinant operators and one single-trace BPS operator, which is the main subject of this paper. The resulting expression is

$$
\begin{aligned}
& \frac{\left\langle\mathcal{D}_{M}\left(x_{1} ; n_{1}, \bar{n}_{1}\right) \mathcal{D}_{M}\left(x_{2} ; n_{2}, \bar{n}_{2}\right) \mathcal{O}_{L}^{\circ}\left(x_{3} ; n_{3}, \bar{n}_{3}\right)\right\rangle}{\mathcal{N}_{\mathcal{D}_{M}} \sqrt{\mathcal{N}_{\mathcal{O}_{L}^{\circ}}}} \\
& \quad=\left(d_{12} d_{21}\right)^{M}\left(\frac{d_{23} d_{32} d_{31} d_{13}}{d_{12} d_{21}}\right)^{\frac{L}{2}} \sum_{p=-\frac{L}{2}}^{\frac{L}{2}} D_{M \mid L}^{(p)} \xi^{p},
\end{aligned}
$$

where $D_{M \mid L}^{(p)}$ 's are structure constants. See (4.53) for the tree-level result for $D_{M \mid L}^{(p)}$.

Non-BPS operators. We now make a few comments on non-BPS operators. In the spinchain approach [34], we start from the vacuum state, which corresponds to the $1 / 3$-BPS operator, and introduce excitations (magnons) in order to describe non-BPS operators. The operators constructed in this way depend on two sets of data;

1. The R-symmetry polarizations ( $n$ and $\tilde{n})$ of the vacuum state $\operatorname{tr}\left[((n \cdot Y)(\bar{n} \cdot \bar{Y}))^{L}\right]$.

2. A set of rapidities of magnons $\mathbf{u}$.

In this paper, we define the normalization of non-BPS operators $\mathcal{N}_{\mathcal{O}}$ in terms of the twopoint function with a canonical choice of the R-symmetry polarizations $n_{0} \equiv(1,0,0,0)$ and $\bar{n}_{0} \equiv(0,0,0,1)$ :

$$
\left\langle\mathcal{O}\left(x_{1} ; n_{0}, \bar{n}_{0}\right)\left[\mathcal{O}\left(x_{2} ; n_{0}, \bar{n}_{0}\right)\right]^{\dagger}\right\rangle=\frac{\mathcal{N}_{\mathcal{O}}}{x_{12}^{2 \Delta_{\mathcal{O}}}}
$$

Here $\mathcal{O}^{\dagger}$ is a Hermitian-conjugate of the operator $\mathcal{O}$ and $\Delta_{\mathcal{O}}$ is the conformal dimension.

We then define the normalized three-point function by

$$
\begin{aligned}
& \frac{\left\langle\mathcal{O}_{1}\left(x_{1} ; n_{1}, \bar{n}_{1}\right) \mathcal{O}_{2}\left(x_{2} ; n_{2}, \bar{n}_{2}\right) \mathcal{O}_{3}\left(x_{3} ; n_{3}, \bar{n}_{3}\right)\right\rangle}{\sqrt{\mathcal{N}_{\mathcal{O}_{1}} \mathcal{N}_{\mathcal{O}_{2}} \mathcal{N}_{\mathcal{O}_{3}}}}
\end{aligned}
$$

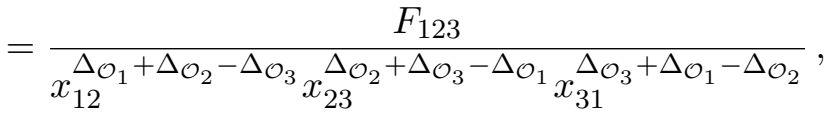


where $F_{123}$ is a sum of all possible allowed $R$-symmetry and Lorentz invariants times the corresponding structure constants. Non-BPS operators typically have more quantum numbers than the BPS operators. Therefore the number of allowed structures for the non-BPS operators is larger than the one for the BPS operators. In addition, the structures highly depend on the Lorentz and the R-symmetry representations of the operator. These features make it difficult to write down a simple universal expression like (2.16). In what follows, we instead focus on special kinematic configurations, called the twisted-translated frame.

Phase ambiguity of structure constants. Note that the normalization of non-BPS operators as defined in (2.23) does not fix the overall phase of the operator $\mathcal{O}$ since a multiplication of a phase to $\mathcal{O}\left(\mathcal{O} \rightarrow e^{i \phi} \mathcal{O}\right)$ does not change $\mathcal{N}_{\mathcal{O}}$. On the other hand, the three-point functions - and therefore structure constants — do change by such a phase multiplication. This means that the overall phase of the structure constants will not be fixed by our analysis ${ }^{9}$ and one needs to impose further conditions in order to determine it. In section 5, we will show that there is one choice of a phase with which the result from integrability takes a simple form. However, this is more like an answer analysis and we do not have a physical explanation on why it simplifies the final expression. It would be important to come up with a field-theory argument on why this choice of a phase is preferred.

\subsection{Twisted translation}

The twisted-translated frame was introduced originally in $\mathcal{N}=4 \mathrm{SYM}$ in four dimensions in [98] and used also in the integrability analysis [7]. A key feature of this frame is that it makes the correlation functions of $1 / 2$ BPS operators position-independent. A similar topological sector in a large class of $\mathcal{N} \geq 4$ SCFTs in three dimensions was studied by localization in [68-71]. For the case of ABJM theory, the full localization results are not yet available ${ }^{10}$, but general symmetry properties of the twisted sector were discussed in [95] and the perturbative computation was performed in [99]. Much like in $\mathcal{N}=4 \mathrm{SYM}$, the twisted-translated frame in ABJM theory provides a useful setup for analyzing the non-BPS three-point functions as well. In fact, it was also discussed in an unpublished work [100, 101] which attempted to construct the hexagon formalism for ABJM theory. As we see below, the structure constant computed in this frame is a particular linear combination of the full structure constants. In principle, we can recover the full structure constants by acting the R-symmetry transformation to each operator, which in the spin-chain language corresponds to adding zero-momentum magnons.

BPS correlation functions. For the correlation functions of BPS operators, the twisted-translated frame is defined by placing all the operators along a single line and aligning the R-symmetry polarizations along an U(1) direction inside SU(4). Written ex-

\footnotetext{
${ }^{9}$ This phase ambiguity was pointed out already in [80].

${ }^{10}$ Two important exceptions are ABJM theory for $k=1[71,72]$ and the correlation function of an operator corresponding to the mass deformation [73-75].
} 
plicitly, the twisted-translated BPS single-trace operator reads

$$
\hat{\mathcal{O}}_{L}^{\circ}(a) \equiv \operatorname{tr}\left[(\mathcal{Y}(a) \overline{\mathcal{Y}}(a))^{L}\right],
$$

where $\mathcal{Y}$ and $\overline{\mathcal{Y}}$ are given by

$$
\begin{aligned}
& \mathcal{Y}(a) \equiv\left(Y^{1}+\kappa a Y^{4}\right)(0, a, 0), \\
& \overline{\mathcal{Y}}(a) \equiv\left(\bar{Y}_{4}-\kappa a \bar{Y}_{1}\right)(0, a, 0) .
\end{aligned}
$$

Here $\kappa$ is an arbitrary parameter with mass dimension 1 . Similarly, the sub-determinant operators in the twisted-translated frame can be defined by

$$
\hat{\mathcal{D}}_{M}(a) \equiv \frac{1}{M !} \delta_{\left[a_{1} \cdots a_{M}\right]}^{\left[b_{1} \cdots b_{M}\right]}(\mathcal{Y}(a) \overline{\mathcal{Y}}(a))_{b_{1}}^{a_{1}} \cdots(\mathcal{Y}(a) \overline{\mathcal{Y}}(a))_{b_{M}}^{a_{M}} .
$$

An alternative way to describe the twisted-translated frame is to use the twisted translation generator, which is a linear combination of the translation and the R-symmetry rotation,

$$
\mathcal{T} \equiv i P_{2}+\kappa R_{1}^{4},
$$

where $P_{2}$ is the translation along the $x_{2}$ direction while $R_{1}^{4}$ is the R-symmetry generator which rotates $Y^{1}$ and $Y^{4}$. Using $\mathcal{T}$, we can express $\mathcal{Y}$ and $\overline{\mathcal{Y}}$ as

$$
\mathcal{Y}(a)=e^{\mathcal{T} a} Y^{1}(0) e^{-\mathcal{T} a}, \quad \overline{\mathcal{Y}}(a)=e^{\mathcal{T} a} \bar{Y}_{4}(0) e^{-\mathcal{T} a} .
$$

An important property of $\mathcal{T}$ is that it is $\mathcal{Q}$ exact, i.e.

$$
\mathcal{T}=\{\mathcal{Q}, \bullet\},
$$

where $\mathcal{Q}$ is a linear combination of the supersymmetry and superconformal generators which is nilpotent and annihilates the $1 / 3$ BPS operator made out of $\mathcal{Y}(0)$ and $\overline{\mathcal{Y}}(0)$. Because of this property, the correlation functions of twisted-translated 1/3 BPS operators (2.25) and (2.26) become independent of the positions $a$ 's and define a topological subsector. See $[95,101]$ for more detailed explanation.

This position independence can also be seen directly from the general structure of BPS two- and three-point functions (2.12), (2.15), (2.16) and (2.22). For this purpose, we simply need to set

$$
n_{j}=\left(1,0,0, \kappa a_{1}\right), \quad \bar{n}_{j}=\left(-\kappa a_{j}, 0,0,1\right), \quad x_{j}=\left(0, a_{j}, 0\right),
$$

in those equations. This leads to $d_{i j}=\kappa \operatorname{sgn}\left(a_{i}-a_{j}\right)$, and we get

$$
\begin{aligned}
& \left\langle\hat{\mathcal{O}}_{L_{1}}^{\circ}\left(a_{1}\right) \hat{\mathcal{O}}_{L_{2}}^{\circ}\left(a_{2}\right)\right\rangle=\delta_{L_{1}, L_{2}} \mathcal{N}_{\mathcal{O}_{L_{1}}^{\circ}}\left(-\kappa^{2}\right)^{L_{1}}, \\
& \left\langle\hat{\mathcal{D}}_{M}\left(a_{1}\right) \hat{\mathcal{D}}_{M}\left(a_{2}\right)\right\rangle=\mathcal{N}_{\mathcal{D}_{M}}\left(-\kappa^{2}\right)^{M},
\end{aligned}
$$

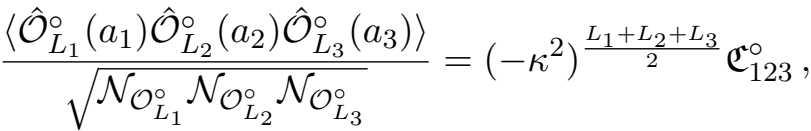

$$
\begin{aligned}
& \frac{\left\langle\hat{\mathcal{D}}_{M}\left(a_{1}\right) \hat{\mathcal{D}}_{M}\left(a_{2}\right) \hat{\mathcal{O}}_{L}^{\circ}\left(a_{3}\right)\right\rangle}{\mathcal{N}_{\mathcal{D}_{M}} \sqrt{\mathcal{N}_{\mathcal{O}_{L}^{\circ}}}}=\left(-\kappa^{2}\right)^{M+\frac{L}{2}} \mathfrak{D}_{M \mid L}^{\circ} .
\end{aligned}
$$


Here $\mathcal{N}_{\mathcal{O}_{L}^{\circ}}$ and $\mathcal{N}_{\mathcal{D}_{M}}$ are normalizations defined in (2.12) and (2.15) while $\mathfrak{C}_{123}^{\circ}$ and $\mathfrak{D}_{M \mid L}^{\circ}$ are the structure constants in the twisted-translated frame, and they are given by linear combinations of the structure constants defined in (2.16) and (2.22):

$$
\mathfrak{C}_{123}^{\circ}=\sum_{p=-\ell_{123}}^{\ell_{123}}(-1)^{p} C_{123}^{(p)}, \quad \mathfrak{D}_{M \mid L}^{\circ}=\sum_{p=-\frac{L}{2}}^{\frac{L}{2}}(-1)^{p} D_{M \mid L}^{(p)} .
$$

Non-BPS operators. To define the twisted-translated frame for non-BPS operators, we first construct a non-BPS operator by adding magnons (denoted in red below) on top of $\operatorname{tr}\left[\left(Y^{1} \bar{Y}_{4}\right)^{L}\right]$ at the origin:

$$
\mathcal{O}(0) \equiv \operatorname{tr}\left[\cdots\left(Y^{1} \bar{Y}_{4}\right)\left(Y^{2} \bar{Y}_{4}\right)\left(Y^{1} \bar{Y}_{3}\right)\left(Y^{1} \bar{Y}_{4}\right) \cdots\right]+\left.\cdots\right|_{x^{\mu}=0} .
$$

As we explain in more detail in the next section, these operators are in one-to-one correspondence with states in the alternating SU(4) invariant spin chain,

$$
\operatorname{tr}\left[\cdots\left(Y^{1} \bar{Y}_{4}\right)\left(Y^{2} \bar{Y}_{4}\right)\left(Y^{1} \bar{Y}_{3}\right)\left(Y^{1} \bar{Y}_{4}\right) \cdots\right] \quad \leftrightarrow \quad|1 \overline{4} 2 \overline{4} 1 \overline{3} 1 \overline{4} \cdots\rangle .
$$

We then act the twisted translation $e^{\mathcal{T} a}$ to obtain an operator at a shifted position:

$$
\hat{\mathcal{O}}(a) \equiv e^{\mathcal{T} a} \mathcal{O}(0) e^{-\mathcal{T} a} .
$$

Note that the twisted translation leaves $Y^{2,3}$ and $\bar{Y}_{2,3}$ invariant:

$$
e^{\mathcal{T} a} Y^{2,3} e^{-\mathcal{T} a}=Y^{2,3}, \quad e^{\mathcal{T} a} \bar{Y}_{2,3} e^{-\mathcal{T} a}=\bar{Y}_{2,3} .
$$

As is clear from the definition above, the twisted-translation only involves an SU(2) subgroup of the full SU(4) R-symmetry. As demonstrated e.g. in appendix A of [102], the $\mathrm{SU}(2)$ Ward identity allows us to express the kinematic dependence of the three-point function in terms of the conformal dimension $\Delta$ and the U(1) R-charge $J$, which assigns $+1 / 2$ charge to $Y^{1}$ and $\bar{Y}_{4}$ and $-1 / 2$ charge to $Y^{4}$ and $\bar{Y}_{1}$. The result reads

$$
\frac{\left\langle\hat{\mathcal{O}}_{1}\left(a_{1}\right) \hat{\mathcal{O}}_{2}\left(a_{2}\right) \hat{\mathcal{O}}_{3}\left(a_{3}\right)\right\rangle}{\sqrt{\mathcal{N}_{\mathcal{O}_{1}} \mathcal{N}_{\mathcal{O}_{2}} \mathcal{N}_{\mathcal{O}_{3}}}}=\frac{\left(-\kappa^{2}\right)^{\frac{J_{1}+J_{2}+J_{3}}{2}} \mathfrak{C}_{123}}{\left(a_{1}-a_{2}\right)^{\gamma_{12 \mid 3}}\left(a_{2}-a_{3}\right)^{\gamma_{23 \mid 1}}\left(a_{3}-a_{1}\right)^{\gamma_{31 \mid 2}}},
$$

with

$$
\gamma_{i j \mid k} \equiv\left(\Delta_{i}-J_{i}\right)+\left(\Delta_{j}-J_{j}\right)-\left(\Delta_{k}-J_{k}\right) .
$$

Here $\mathcal{N}_{\mathcal{O}}$ is the normalization defined in (2.23), and $\mathfrak{C}_{123}$ is the structure constant in the twisted-translated frame, which is $F_{123}$ in $(2.24)$ specialized to the twisted-translated kinematics.

These expressions can be generalized to the correlation function of two sub-determinant operators and a single-trace non-BPS operator. The result reads

$$
\frac{\left\langle\hat{\mathcal{D}}_{M}\left(a_{1}\right) \hat{\mathcal{D}}_{M}\left(a_{2}\right) \hat{\mathcal{O}}\left(a_{3}\right)\right\rangle}{\mathcal{N}_{\mathcal{D}_{M}} \sqrt{\mathcal{N}_{\mathcal{O}}}}=\left(-\kappa^{2}\right)^{M+\frac{J}{2}}\left(\frac{\left(a_{1}-a_{2}\right)}{\left(a_{2}-a_{3}\right)\left(a_{3}-a_{1}\right)}\right)^{\Delta-J} \mathfrak{D}_{M \mid \mathcal{O}},
$$

with $\mathfrak{D}_{M \mid \mathcal{O}}$ being the structure constant. The main goal of this paper is to compute $\mathfrak{D}_{M \mid \mathcal{O}}$ at tree level using the spin-chain description. 


\section{$3 \quad \mathrm{SU}(4)$ invariant alternating spin chain}

In this section, we explain the spin-chain description of single-trace operators made out of scalar fields. After reviewing the basic facts of the SU(4) invariant alternating spin chain and its relation to single-trace operators, we discuss its coordinate Bethe ansatz. In particular, we present explicit expressions for the coordinate Bethe ansatz wave functions, which we will use later to evaluate the structure constants (2.42). An alternative way to construct the wave function is to use the algebraic Bethe ansatz which we review in appendix D.

\subsection{Hamiltonian and Bethe equations}

The dilatation operator in the scalar sector of ABJM theory at two-loop order is described by the SU(4) invariant alternating spin chain [34, 37]. The Hamiotnonian of the spin chain is given by

$$
\mathbb{H}=\frac{\lambda^{2}}{2} \sum_{l=1}^{2 L}\left(2-2 \mathrm{P}_{l, l+2}+\mathrm{P}_{l, l+2} \mathrm{~K}_{l, l+1}+\mathrm{K}_{l, l+1} \mathrm{P}_{l, l+2}\right)
$$

where $\mathrm{P}_{a b}$ and $\mathrm{K}_{a b}$ are permutation and trace operators acting on the $a$-th and $b$-th sites. We denote the set of orthonormal basis of the Hilbert space at each site by $|i\rangle, i=1, \cdots, 4$. The two operators act as

$$
\mathrm{P}|i\rangle \otimes|j\rangle=|j\rangle \otimes|i\rangle, \quad \mathrm{K}|i\rangle \otimes|j\rangle=\delta_{i j} \sum_{k=1}^{4}|k\rangle \otimes|k\rangle .
$$

Using these definitions, it is straightforward to show that $\mathrm{P}_{a b}$ and $\mathrm{K}_{a b}$ obey the following relations

$$
\mathrm{P}_{a b} \mathrm{P}_{a b}=1, \quad \mathrm{~K}_{a b} \mathrm{~K}_{a b}=4 \mathrm{~K}_{a b}, \quad \mathrm{P}_{a b} \mathrm{~K}_{b c}=\mathrm{K}_{a c} \mathrm{P}_{a b}=\mathrm{K}_{a c} \mathrm{~K}_{b c} .
$$

The spin chain under consideration is an alternating spin chain. The odd and even sites sit in the $\mathbf{4}$ and $\overline{\mathbf{4}}$ representation of SU(4) group and correspond to fields $Y^{A}$ and $\bar{Y}_{A}$ in ABJM theory respectively. In order to distinguish odd and even sites, we put bars to the odd sites, namely

$$
Y^{A} \mapsto|A\rangle, \quad \bar{Y}_{A} \mapsto|\bar{A}\rangle
$$

In this notation, the single-trace operator $\operatorname{tr}\left(Y^{A_{1}} \bar{Y}_{B_{1}} Y^{A_{2}} \bar{Y}_{B_{2}} \cdots\right)$ is mapped to the following spin-chain state;

$$
\operatorname{tr}\left(Y^{A_{1}} \bar{Y}_{B_{1}} Y^{A_{2}} \bar{Y}_{B_{2}} \cdots\right) \quad \mapsto\left|A_{1} \bar{B}_{1} A_{2} \bar{B}_{2} \cdots\right\rangle .
$$

One special feature of the Hamiltonian $((3.1))$ is that the permutation operator $\mathrm{P}_{l, l+2}$ only act on the odd- or even-site spin chains while the trace operator $\mathrm{K}_{l, l+1}$ only mix the two. We have

$$
\mathrm{K}|A\rangle \otimes|\bar{B}\rangle=\delta_{A B} \sum_{C=1}^{4}|C\rangle \otimes|\bar{C}\rangle .
$$




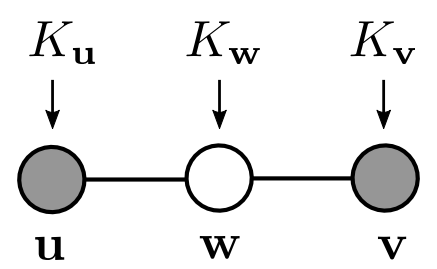

Figure 1. Dynkin diagram of the SU(4) alternating spin chain. The gray circles are the momentum carrying nodes.

The Hamiltonian (3.1) is known to be integrable and solvable by the Bethe ansatz. Reflecting the alternating strucrure of the spin chain, the Bethe state is described by two sets of the momentum carrying Bethe roots which we denote by $\mathbf{u}$ and $\mathbf{v}$ (see figure 1). In addition, there are auxiliary Bethe roots $\mathbf{w}$. The numbers of rapidities of $\mathbf{u}, \mathbf{v}, \mathbf{w}$ are denoted by $K_{\mathbf{u}}, K_{\mathbf{v}}, K_{\mathbf{w}}$ respectively. The rapidities satisfy the Bethe equations

$$
\begin{aligned}
& 1=e^{i \phi_{u_{j}}}=\left(\frac{u_{j}+\frac{i}{2}}{u_{j}-\frac{i}{2}}\right)^{L} \prod_{\substack{k=1 \\
k \neq j}}^{K_{\mathbf{u}}} S\left(u_{j}, u_{k}\right) \prod_{k=1}^{K_{\mathbf{w}}} \tilde{S}\left(u_{j}, w_{k}\right), \\
& 1=e^{i \phi_{w_{j}}}=\prod_{\substack{k=1 \\
k \neq j}}^{K_{\mathbf{w}}} S\left(w_{j}, w_{k}\right) \prod_{k=1}^{K_{\mathbf{u}}} \tilde{S}\left(w_{j}, u_{k}\right) \prod_{k=1}^{K_{\mathbf{v}}} \tilde{S}\left(w_{j}, v_{k}\right), \\
& 1=e^{i \phi_{v_{j}}}=\left(\frac{v_{j}+\frac{i}{2}}{v_{j}-\frac{i}{2}}\right)^{L} \prod_{\substack{k=1 \\
k \neq j}}^{K_{\mathbf{v}}} S\left(v_{j}, v_{k}\right) \prod_{k=1}^{K_{\mathbf{w}}} \tilde{S}\left(v_{j}, w_{k}\right),
\end{aligned}
$$

where the S-matrices $S(u, v)$ and $\tilde{S}(u, v)$ are given by

$$
S(u, v) \equiv \frac{u-v-i}{u-v+i}, \quad \tilde{S}(u, v) \equiv \frac{u-v+\frac{i}{2}}{u-v-\frac{i}{2}}
$$

The two-loop anomalous dimension of the operator is given by

$$
\Delta-L=\lambda^{2}\left(\sum_{k=1}^{K_{\mathbf{u}}} \frac{1}{u_{k}^{2}+\frac{1}{4}}+\sum_{k=1}^{K_{\mathbf{v}}} \frac{1}{v_{k}^{2}+\frac{1}{4}}\right) .
$$

The U(1) R-charge $J$ discussed in section 2.3 can be expressed in terms of $K_{\mathbf{u}, \mathbf{v}}$ as

$$
J=L-\frac{K_{\mathbf{u}}+K_{\mathbf{v}}}{2} .
$$

Zero-momentum condition. As is the case with $\mathcal{N}=4 \mathrm{SYM}$, not all solutions to the Bethe equation corresponds to a single-trace operator in ABJM theory. This is because the single-trace operator has an additional cyclicity property. In the spin-chain language, this is equivalent to the zero-momentum condition,

$$
1=\prod_{j=1}^{K_{\mathbf{u}}} \frac{u_{j}+\frac{i}{2}}{u_{j}-\frac{i}{2}} \prod_{j=1}^{K_{\mathrm{v}}} \frac{v_{j}+\frac{i}{2}}{v_{j}-\frac{i}{2}} .
$$




\subsection{Coordinate Bethe ansatz}

The eigenvector of the spin chain (3.1) can be constructed by the nested coordinate Bethe ansatz (CBA). To describe the CBA construction, we map each scalar field to a specific combination of the Bethe roots following the notation in appendix E of [10]. More precisely, we first express odd- and even-sites by bullets $\bullet$ and circles o respectively, and place Bethe roots on top of them. Then, the relation between stacks of Bethe roots and the fields in ABJM theory is given by

$$
\begin{aligned}
& Y^{1} \mapsto|1\rangle=|\bullet\rangle, \quad Y^{2} \mapsto|2\rangle=|\bullet\rangle, \quad Y^{3} \mapsto|3\rangle=|\stackrel{w}{u}\rangle, \quad Y^{4} \mapsto|4\rangle=\left|\stackrel{w}{w} \bullet^{u}\right\rangle,
\end{aligned}
$$

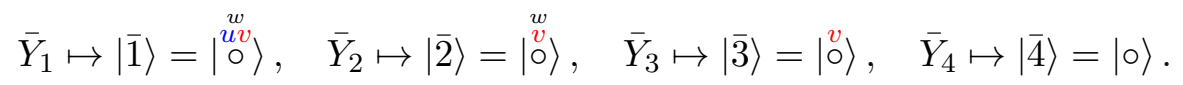

We now briefly outline the procedure of constructing eigenvectors. It can be achieved in two steps. In the first step, we distribute the rapidities on different sites of the spin chain and sum over all such possibilities. In the second step, we construct the wave function for each distribution and multiply it to the corresponding distribution. Plugging in the physical solutions of the Bethe roots, we obtain an eigenvector of the Hamiltonian.

Distributing excitations. We consider the spin chain of length $2 L$. We first distribute these rapidities on different sites by the following procedure

1. Firstly we distribute the momentum carrying rapidities $\left\{u_{1}, \cdots, u_{K_{\mathbf{u}}}\right\}$ and $\left\{v_{1}, \cdots, v_{K_{\mathrm{v}}}\right\}$ on top of the ground state $\left|\bullet_{1}, \circ_{1}, \cdots \bullet_{L}, \circ_{L}\right\rangle$. Each odd site can support either a single $u$-type rapidity, or two rapdities one of $u v$-type. Likewise, each even site can support a single $v$-type rapidity, or two rapidities of $u v$-type.

2. Now we view the physical rapidities $\left\{u_{1}, u_{2}, \cdots, u_{K_{\mathbf{u}}}\right\}$ and $\left\{v_{1}, v_{2}, \cdots, v_{K_{\mathbf{v}}}\right\}$ as inhomogeneities of an emergent spin chain of length $K_{\mathbf{u}}+K_{\mathbf{v}}$ and distribute the rapidities $\left\{w_{1}, w_{2}, \cdots, w_{K_{\mathbf{w}}}\right\}$ on the emergent chain. Each $w_{j}$ can be distributed on top of either $u_{i}$ or $v_{i}$.

3. In the previous two steps, we also generate string configurations without field theory correspondence in (3.12). For example, $\bullet^{\bullet v}$ does not corresponds to any of the fields $Y^{A}$ or $\bar{Y}_{A}$. We set all the states which contain such string configurations to zero.

We give an example of the procedure described above in figure 2 .

In what follows, we denote the stack of Bethe roots at site $n$ by $\mathrm{s}_{n}$. The possible configurations are

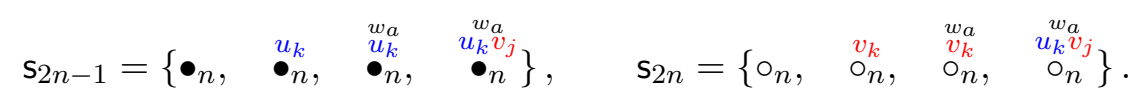

A collection of all $\mathbf{s}_{n}$ 's for a given distribution will be denoted by $\vec{s}$

$$
\overrightarrow{\mathrm{s}} \equiv\left\{\mathrm{s}_{1}, \mathrm{~s}_{2}, \ldots, \mathrm{s}_{2 L-1}, \mathrm{~s}_{2 L}\right\}
$$




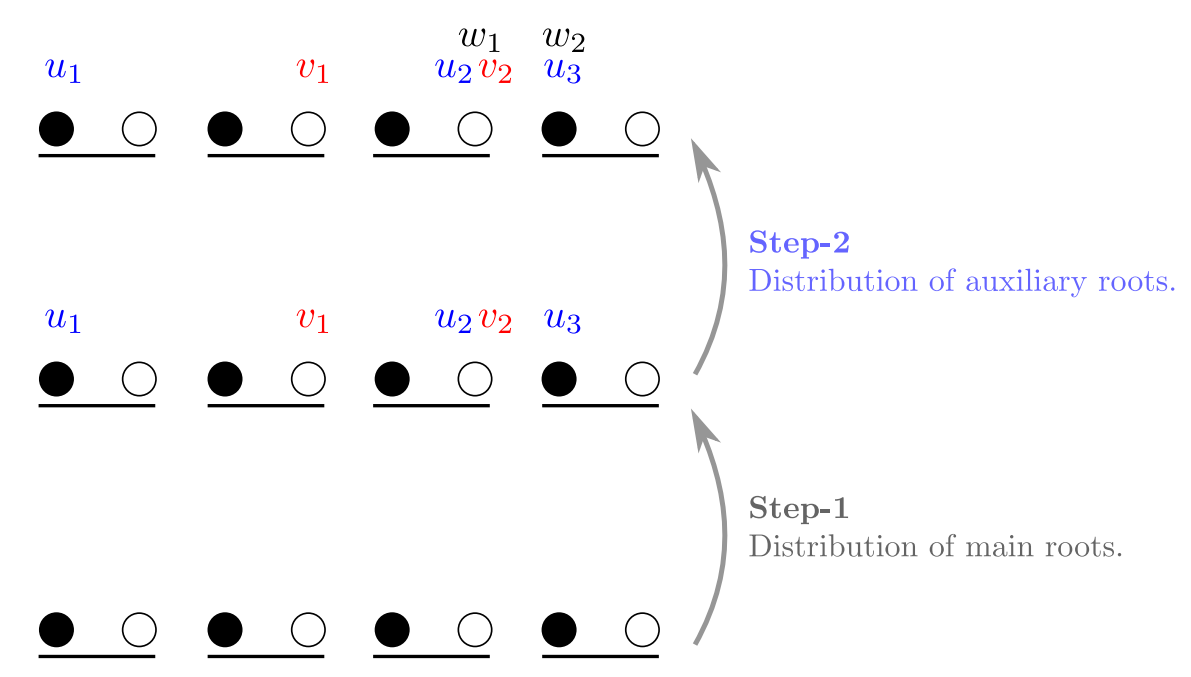

Figure 2. Construction of (one of) the Bethe string configuration corresponding to the ket state $\operatorname{tr}\left(Y^{2} \bar{Y}_{4}\right)\left(Y^{1} \bar{Y}_{3}\right)\left(Y^{1} \bar{Y}_{1}\right)\left(Y^{3} \bar{Y}_{4}\right)=|2, \overline{4}, 1, \overline{3}, 1, \overline{1}, 3, \overline{4}\rangle$.

The wave functions: ordered configurations. The next step is to write down a wave function for each distribution of rapidities. We first discuss the distribution in which the rapidities are in the canonical order (namely in the same order as $\mathbf{u}, \mathbf{v}$ and $\mathbf{w}$ ). We call such distributions ordered distributions. The wave function for a ordered distribution of Bethe roots $\vec{s}$ is given by a product of "single-site wave functions" $\Phi(\mathrm{s})$;

$$
\Psi_{\overrightarrow{\mathbf{s}}}(\mathbf{u}, \mathbf{v}, \mathbf{w})=\prod_{n=1}^{2 L} \Phi\left(\mathbf{s}_{n}\right) .
$$

The single-site wave functions with at most a single rapidity are

$$
\Phi\left(\bullet_{n}\right)=\Phi\left(\circ_{n}\right)=1, \quad \Phi\left(\bullet_{n}^{u_{k}}\right)=\left(\frac{u_{k}+\frac{i}{2}}{u_{k}-\frac{i}{2}}\right)^{n}, \quad \Phi\left(\circ_{n}^{v_{k}}\right)=\left(\frac{v_{k}+\frac{i}{2}}{v_{k}-\frac{i}{2}}\right)^{n} .
$$

It is slightly more complicated to write a single-site wave function when there is $w$ because the expression depends on the Bethe roots on other sites; or more precisely on the momentum carrying rapidities to the left of the site. For instance, $\Phi(\mathrm{s})$ 's corresponding to $\left\{\stackrel{w}{u} \stackrel{w}{v}_{n}, o_{n}\right\}$ are given by

$$
\begin{aligned}
& \Phi\left(\stackrel{\bullet_{a}^{w_{k}}}{\mathfrak{w}_{n}}\right)=\left(\frac{u_{k}+\frac{i}{2}}{u_{k}-\frac{i}{2}}\right)^{n} \times \psi\left(w_{a} \mid \boldsymbol{z}^{<}\right) \times \frac{-1}{w_{a}-u_{k}-\frac{i}{2}}, \\
& \Phi\left(\begin{array}{c}
w_{a} \\
\mathrm{o}_{k} \\
n
\end{array}\right)=\left(\frac{v_{k}+\frac{i}{2}}{v_{k}-\frac{i}{2}}\right)^{n} \times \psi\left(w_{a} \mid \boldsymbol{z}^{<}\right) \times \frac{1}{w_{a}-v_{k}-\frac{i}{2}},
\end{aligned}
$$

where $\boldsymbol{z}^{<}$denotes all the momentum carrying rapidities that are to the left of the site which supports $w$. The explicit form is given by

$$
\psi\left(w \mid \boldsymbol{z}^{<}\right)=\prod_{j} \frac{w-z_{j}^{<}+\frac{i}{2}}{w-z_{j}^{<}-\frac{i}{2}} .
$$


Similarly, the single-site wave functions for the configurations $\left\{\stackrel{w v}{\mathfrak{u}_{n},{ }^{w v}}{ }_{n}^{w}\right\}$ are given by

$$
\begin{aligned}
& \left.\begin{array}{c}
w_{a}^{w_{a}} \\
u_{k} v_{j}
\end{array}\right)=\left(\frac{u_{k}+\frac{i}{2}}{u_{k}-\frac{i}{2}}\right)^{n}\left(\frac{v_{j}+\frac{i}{2}}{v_{j}-\frac{i}{2}}\right)^{n} \times \psi\left(w_{a} \mid \boldsymbol{z}^{<}\right) \times \frac{-\left(v_{j}-\frac{i}{2}\right)}{\left(w_{a}-u_{k}-\frac{i}{2}\right)\left(w_{a}-v_{j}-\frac{i}{2}\right)}, \\
\Phi\left(\begin{array}{c}
w_{k} v_{j} \\
{ }^{w_{n}}
\end{array}\right) & =\left(\frac{u_{k}+\frac{i}{2}}{u_{k}-\frac{i}{2}}\right)^{n}\left(\frac{v_{k}+\frac{i}{2}}{v_{k}-\frac{i}{2}}\right)^{n} \times \psi\left(w_{a} \mid \boldsymbol{z}^{<}\right) \times \frac{+\left(u_{k}+\frac{i}{2}\right)}{\left(w_{a}-u_{k}-\frac{i}{2}\right)\left(w_{a}-v_{j}-\frac{i}{2}\right)} .
\end{aligned}
$$

The wave functions: general configurations. More general configurations of the rapidities can be obtained from the ordered configurations by performing permutations of the Bethe roots. For such configuraions, the wave function consists of two factors. The first factor is a product of single-site wave functions $\Phi$,

$$
\prod_{n=1}^{2 L} \Phi\left(\mathbf{s}_{n}\right)
$$

The second factor is a product of S-matrices, which are needed to bring the rapidities in the distribution into the canonical order. For instance, for the distribution in which $u_{2}$ is to the left of $u_{1}$, we need to multiply

$$
S\left(u_{1}, u_{2}\right)=\frac{u_{1}-u_{2}-i}{u_{1}-u_{2}+i} .
$$

We multiply similar factors $\left(S\left(v_{j}, v_{k}\right)\right.$ and $\left.S\left(w_{j}, w_{k}\right)\right)$ also for $v$ 's and $w$ 's. Therefore, the wave function for a general distribution is given by

$$
\Psi_{\overrightarrow{\mathbf{s}}}(\mathbf{u}, \mathbf{v}, \mathbf{w})=\mathbb{S} \times \prod_{n=1}^{2 L} \Phi\left(\mathbf{s}_{n}\right),
$$

where $\mathbb{S}$ is a product of S-matrices described above.

The Bethe eigenstate is given by a summation of all possible distributions; namely

$$
\left|\Psi_{\mathbf{u}, \mathbf{v}, \mathbf{w}}\right\rangle=\sum_{\overrightarrow{\overrightarrow{\mathbf{s}} \in} \in{ }_{\text {distributions }}^{\text {all possible }}} \Psi_{\overrightarrow{\mathbf{s}}}(\mathbf{u}, \mathbf{v}, \mathbf{w})|\overrightarrow{\mathbf{s}}\rangle .
$$

Examples. As explicit examples of the procedure described above, we give three simple states for $L=2$. The vacuum state is given by

$$
|\Omega\rangle=|1, \overline{4}, 1, \overline{4}\rangle .
$$

First, we consider the state with $K_{\mathbf{u}}=1, K_{\mathbf{v}}=1, K_{\mathbf{w}}=0$. The state is given by a sum of four terms, and below we list them along with the wave function for each distribution:

$$
\begin{aligned}
& |2, \overline{3}, 1, \overline{4}\rangle \equiv\left|\bullet_{1}, \stackrel{v}{\circ}_{1}, \bullet_{2}, \circ_{2}\right\rangle \quad\left(\frac{u+\frac{i}{2}}{u-\frac{i}{2}}\right)\left(\frac{v+\frac{i}{2}}{v-\frac{i}{2}}\right), \\
& |2, \overline{4}, 1, \overline{3}\rangle \equiv\left|\bullet_{1}, \circ_{1}, \bullet_{2}, \stackrel{\circ}{\circ}_{2}\right\rangle \quad\left(\frac{u+\frac{i}{2}}{u-\frac{i}{2}}\right)\left(\frac{v+\frac{i}{2}}{v-\frac{i}{2}}\right)^{2}, \\
& |1, \overline{3}, 2, \overline{4}\rangle \equiv\left|\bullet_{1}, \stackrel{v}{\circ}_{1}, \bullet_{2}, \circ_{2}\right\rangle \quad\left(\frac{u+\frac{i}{2}}{u-\frac{i}{2}}\right)^{2}\left(\frac{v+\frac{i}{2}}{v-\frac{i}{2}}\right), \\
& |1, \overline{4}, 2, \overline{3}\rangle \equiv\left|\bullet_{1}, \circ_{1}, \stackrel{u}{u}, \stackrel{v}{\circ}_{2}\right\rangle \quad\left(\frac{u+\frac{i}{2}}{u-\frac{i}{2}}\right)^{2}\left(\frac{v+\frac{i}{2}}{v-\frac{i}{2}}\right)^{2} .
\end{aligned}
$$


Second, we consider the state with $K_{\mathbf{u}}=2, K_{\mathbf{v}}=K_{\mathbf{w}}=0$. It consists of the following two terms

$$
\begin{aligned}
& |2, \overline{4}, 2, \overline{4}\rangle_{1} \equiv\left|\bullet_{1}, \circ_{1}, \stackrel{u}{2}_{2}, \circ_{2}\right\rangle \quad\left(\frac{u_{1}+\frac{i}{2}}{u_{1}-\frac{i}{2}}\right)\left(\frac{u_{2}+\frac{i}{2}}{u_{2}-\frac{i}{2}}\right)^{2}, \\
& |2, \overline{4}, 2, \overline{4}\rangle_{2} \equiv\left|\bullet_{1}^{u_{2}}, \circ_{1}, \bullet_{2}^{u_{1}}, \circ_{2}\right\rangle \quad \frac{u_{1}-u_{2}-i}{u_{1}-u_{2}+i}\left(\frac{u_{2}+\frac{i}{2}}{u_{2}-\frac{i}{2}}\right)\left(\frac{u_{1}+\frac{i}{2}}{u_{1}-\frac{i}{2}}\right)^{2} .
\end{aligned}
$$

The first term is an ordered distribution while the second term is not. This is why the second term comes with an extra factor of the S-matrix $S\left(u_{1}, u_{2}\right)=\left(u_{1}-u_{2}-i\right) /\left(u_{1}-u_{2}+i\right)$.

As the third example, we consider the state with $K_{\mathbf{u}}=1, K_{\mathbf{v}}=1, K_{\mathbf{w}}=1$. In this case, there are 12 terms, which we list below

$$
\begin{aligned}
& |2, \overline{2}, 1, \overline{4}\rangle \equiv\left|\bullet_{1}, \stackrel{w}{w} \stackrel{\circ}{1}_{1}, \bullet_{2}, \circ_{2}\right\rangle \quad\left(\frac{u+\frac{i}{2}}{u-\frac{i}{2}}\right)\left(\frac{v+\frac{i}{2}}{v-\frac{i}{2}}\right) \frac{w-u+\frac{i}{2}}{w-u-\frac{i}{2}} \frac{1}{w-v-\frac{i}{2}}, \\
& |2, \overline{4}, 1, \overline{2}\rangle \equiv\left|\bullet_{1}, \circ_{1}, \bullet_{2}, \stackrel{w}{v}_{2}\right\rangle \quad\left(\frac{u+\frac{i}{2}}{u-\frac{i}{2}}\right)\left(\frac{v+\frac{i}{2}}{v-\frac{i}{2}}\right)^{2} \frac{w-u+\frac{i}{2}}{w-u-\frac{i}{2}} \frac{1}{w-v-\frac{i}{2}}, \\
& |1, \overline{2}, 2, \overline{4}\rangle \equiv\left|\bullet_{1}, \stackrel{v}{w}_{1}^{w}, \bullet_{2}, \circ_{2}\right\rangle \quad\left(\frac{u+\frac{i}{2}}{u-\frac{i}{2}}\right)^{2}\left(\frac{v+\frac{i}{2}}{v-\frac{i}{2}}\right) \frac{1}{w-v-\frac{i}{2}}, \\
& |1, \overline{4}, 2, \overline{2}\rangle \equiv\left|\bullet_{1}, \circ_{1}, \bullet_{2}^{u}, \stackrel{v}{v}_{2}^{w}\right\rangle \quad\left(\frac{u+\frac{i}{2}}{u-\frac{i}{2}}\right)^{2}\left(\frac{v+\frac{i}{2}}{v-\frac{i}{2}}\right)^{2} \frac{w-u+\frac{i}{2}}{w-u-\frac{i}{2}} \frac{1}{w-v-\frac{i}{2}}, \\
& |3, \overline{3}, 1, \overline{4}\rangle \equiv\left|\bullet_{1}^{w}, \circ_{1}^{v}, \bullet_{2}, \circ_{2}\right\rangle \quad\left(\frac{u+\frac{i}{2}}{u-\frac{i}{2}}\right)\left(\frac{v+\frac{i}{2}}{v-\frac{i}{2}}\right) \frac{-1}{w-u-\frac{i}{2}}, \\
& \left.\left.|3, \overline{4}, 1, \overline{3}\rangle \equiv\right|_{\bullet} ^{w}, \circ_{1}, \bullet_{2}, \stackrel{v}{\circ}_{2}\right\rangle \quad\left(\frac{u+\frac{i}{2}}{u-\frac{i}{2}}\right)\left(\frac{v+\frac{i}{2}}{v-\frac{i}{2}}\right)^{2} \frac{-1}{w-u-\frac{i}{2}}, \\
& |1, \overline{4}, 3, \overline{3}\rangle \equiv\left|\bullet_{1}, \circ_{1}, \stackrel{w}{\bullet} \bullet_{2}, \stackrel{v}{\circ_{2}}\right\rangle \quad\left(\frac{u+\frac{i}{2}}{u-\frac{i}{2}}\right)^{2}\left(\frac{v+\frac{i}{2}}{v-\frac{i}{2}}\right)^{2} \frac{-1}{w-u-\frac{i}{2}},
\end{aligned}
$$

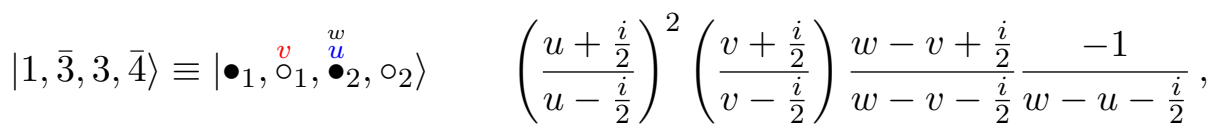

$$
\begin{aligned}
& |4, \overline{4}, 1, \overline{4}\rangle \equiv\left|\bullet_{1}^{w}, \circ_{1}, \bullet_{2}, \circ_{2}\right\rangle \quad\left(\frac{u+\frac{i}{2}}{u-\frac{i}{2}}\right)\left(\frac{v+\frac{i}{2}}{v-\frac{i}{2}}\right) \frac{-\left(v-\frac{i}{2}\right)}{\left(w-v-\frac{i}{2}\right)\left(w-u-\frac{i}{2}\right)}, \\
& |1, \overline{1}, 1, \overline{4}\rangle \equiv\left|\bullet_{1}, \stackrel{w}{\sim_{0}}{ }_{1}, \bullet_{2}, \circ_{2}\right\rangle \quad\left(\frac{u+\frac{i}{2}}{u-\frac{i}{2}}\right)\left(\frac{v+\frac{i}{2}}{v-\frac{i}{2}}\right) \frac{+\left(u+\frac{i}{2}\right)}{\left(w-v-\frac{i}{2}\right)\left(w-u-\frac{i}{2}\right)}, \\
& |1, \overline{4}, 4, \overline{1}\rangle \equiv\left|\bullet_{1}, \circ_{1}, \stackrel{w}{u v}_{2}, \circ_{2}\right\rangle \quad\left(\frac{u+\frac{i}{2}}{u-\frac{i}{2}}\right)^{2}\left(\frac{v+\frac{i}{2}}{v-\frac{i}{2}}\right)^{2} \frac{-\left(v-\frac{i}{2}\right)}{\left(w-v-\frac{i}{2}\right)\left(w-u-\frac{i}{2}\right)}, \\
& |1, \overline{4}, 1, \overline{1}\rangle \equiv\left|\bullet_{1}, \circ_{1}, \bullet_{2}, \stackrel{w}{w v}_{2}\right\rangle \quad\left(\frac{u+\frac{i}{2}}{u-\frac{i}{2}}\right)^{2}\left(\frac{v+\frac{i}{2}}{v-\frac{i}{2}}\right)^{2} \frac{+\left(u+\frac{i}{2}\right)}{\left(w-v-\frac{i}{2}\right)\left(w-u-\frac{i}{2}\right)} \text {. }
\end{aligned}
$$




\section{Matrix product states from giant gravitons}

To compute the structure constant (2.42) using integrability, we first need to map it to a quantity in the spin chain. For $\mathcal{N}=4 \mathrm{SYM}$, this was done ${ }^{11}$ in $[29,30,59]$, which showed that the structure constant (2.42) corresponds to an overlap between a matrix product state (MPS) and a Bethe eigenstate. This analysis was later generalized to ABJ(M) theory in [77]. In this section, we review and extend the results in [77]. Two main new results are

1. We compute tree-level structure constants of two non-maximal giant gravitons and a single-trace BPS operator.

2. We derive an explicit expression for MPS of two non-maximal giant gravitons in the twisted-translated frame which can be readily used in later sections.

\subsection{Derivation of matrix product states}

Although the main goal of this paper is to compute the structure constant in the twistedtranslated frame (2.42), in this subsection we consider a slightly more general setup; the correlation function of $m$ giant gravitons and a single-trace operator in a general kinematics. More precisely we consider the following correlation function

$$
G_{m}=\left\langle\left(\prod_{j=1}^{m} \mathcal{G}_{j}\right) \mathcal{O}(y)\right\rangle
$$

where $\mathcal{G}_{j}$ is a generating function for giant gravitons (cf. (2.10)):

$$
\mathcal{G}_{j} \equiv \operatorname{det}\left(\begin{array}{cc}
\mathbf{1} & -t_{j}\left(\bar{n}_{j} \cdot \bar{Y}\right) \\
t_{j}\left(n_{j} \cdot Y\right) & \mathbf{1}
\end{array}\right)\left(x_{j}\right) .
$$

To extract the correlation functions of individual giant gravitons, we simply need to perform integrals of $t_{j}$ 's,

$$
\left\langle\left(\prod_{j=1}^{m} \mathcal{D}_{M_{j}}\right) \mathcal{O}(y)\right\rangle=\left(\prod_{j=1}^{m} \oint \frac{d t_{j}}{t_{j}^{1+2 M_{j}}}\right) G_{m}
$$

Since giant gravitons carry large R-charges $M_{j} \sim \mathcal{O}(N)$, most of these integrals in the large $N$ limit can be computed by the saddle-point approximation. We will later see how this works in practice. For now we focus on computing the correlation functions of generating functions.

Let us now compute $G_{m}$ at tree level. This is given by the following path integral

$$
G_{m}=\frac{1}{Z_{Y}} \int D Y^{I} D \bar{Y}_{I}\left(\prod_{j=1}^{m} \mathcal{G}_{j}\right) \mathcal{O} \exp \left[-\frac{k}{4 \pi} \int \mathrm{d}^{3} x \operatorname{tr}\left(\partial_{\mu} \bar{Y}_{I} \partial^{\mu} Y^{I}\right)\right],
$$

\footnotetext{
${ }^{11}$ The analysis was recently generalized to the four-point function of determinant operators in $\mathcal{N}=4$ SYM [103].
} 
with

$$
Z_{Y}=\int D Y^{I} D \bar{Y}_{I} \exp \left[-\frac{k}{4 \pi} \int \mathrm{d}^{3} x \operatorname{tr}\left(\partial_{\mu} \bar{Y}_{I} \partial^{\mu} Y^{I}\right)\right]
$$

The generating functions can be written in terms of path integral of fermions

$$
\mathcal{G}_{j}=\int d \eta_{j} d \bar{\eta}_{j} d \chi_{j} d \bar{\chi}_{j} \exp \left[\left(\bar{\chi}_{j} \chi_{j}\right)+\left(\bar{\eta}_{j} \eta_{j}\right)+t_{j} \bar{\chi}_{j}\left(n_{j} \cdot Y\right) \eta_{j}-t_{j} \bar{\eta}_{j}\left(\bar{n}_{j} \cdot \bar{Y}\right) \chi_{j}\right] .
$$

Here the fermions $\chi_{i}$ and $\eta_{i}$ are in the fundamental representation of the gauge group $\mathrm{U}(N)_{k}$ and $\mathrm{U}(N)_{-k}$ respectively while $\bar{\chi}_{i}, \bar{\eta}_{i}$ are in the antifundamental representation. The indices are contracted as

$$
\bar{\chi}(n \cdot Y) \eta=\bar{\chi}^{a}(n \cdot Y)_{a}{ }^{\bar{b}} \eta_{\bar{b}}, \quad \bar{\eta}(\bar{n} \cdot \bar{Y}) \chi=\bar{\eta}^{\bar{a}}(\bar{n} \cdot \bar{Y})_{\bar{a}}{ }^{b} \chi_{b} .
$$

We can then rewrite the correlation function $G_{m}$ as

$$
G_{m}=\frac{1}{Z_{Y}} \int D Y^{I} D \bar{Y}_{I} \prod_{i=1}^{m} d \bar{\chi}_{i} d \eta_{i} d \bar{\eta}_{i} d \chi_{i} \mathcal{O} \exp \left(-S_{\chi, \eta}\right)
$$

where

$$
\begin{aligned}
S_{\chi, \eta}= & -\sum_{i=1}^{m}\left(\left(\bar{\eta}_{i} \eta_{i}\right)+\left(\bar{\chi}_{i} \chi_{i}\right)\right)+\frac{k}{4 \pi} \int \mathrm{d}^{3} x\left[\operatorname{tr}\left(\partial_{\mu} \bar{Y}_{I} \partial^{\mu} Y^{I}\right)\right. \\
& \left.-\frac{4 \pi}{k} \sum_{i=1}^{m} t_{i} \bar{\chi}_{i}\left(n_{i} \cdot Y\right) \eta_{i} \delta^{3}\left(x-x_{i}\right)+\frac{4 \pi}{k} \sum_{i=1}^{m} t_{i} \bar{\eta}_{i}\left(\bar{n}_{i} \cdot \bar{Y}\right) \chi_{i} \delta^{3}\left(x-x_{i}\right)\right] .
\end{aligned}
$$

Since this action is quadratic in $Y^{I}, \bar{Y}_{I}$, we can integrate them out. In practice, this is equivalent to solving the equations of motion for these fields,

$$
\begin{aligned}
& \square\left(Y^{I}\right)_{a}^{\bar{b}}(x)=-\frac{4 \pi}{k} \sum_{i=1}^{m} t_{i} \bar{n}_{i}^{I} \chi_{i, a} \bar{\eta}_{i}^{\bar{b}} \delta^{3}\left(x-x_{i}\right), \\
& \square\left(Y_{I}^{\dagger}\right)_{\bar{a}}^{b}(x)=\frac{4 \pi}{k} \sum_{i=1}^{m} t_{i} n_{i, I} \eta_{i, \bar{a}} \bar{\chi}_{i}^{b} \delta^{3}\left(x-x_{i}\right) .
\end{aligned}
$$

These equations can be solved by Green's function. Using the fact that

$$
\square \frac{1}{|x-y|}=-4 \pi \delta^{3}(x-y),
$$

the solution to (4.10) is given by

$$
Y^{I}(x)=S^{I}(x) \equiv \frac{1}{k} \sum_{i=1}^{m} \frac{t_{i} \bar{n}_{i}^{I} \chi_{i} \bar{\eta}_{i}}{\left|x-x_{i}\right|}, \quad \bar{Y}_{I}(x)=\bar{S}_{I}(x) \equiv-\frac{1}{k} \sum_{i=1}^{m} \frac{t_{i} n_{i, I} \eta_{i} \bar{\chi}_{i}}{\left|x-x_{i}\right|} .
$$

Plugging the solution (4.12) back to (4.8), we obtain

$$
G_{m}=\int \prod_{i=1}^{m} d \eta_{i} d \bar{\eta}_{i} d \chi_{i} d \bar{\chi}_{i} \mathcal{O}^{S} \exp \left(-\widetilde{S}_{\chi, \eta}\right)
$$


where $\mathcal{O}^{S}$ is obtained by replacing all $Y^{I}, \bar{Y}_{I}$ by their classical solution $S^{I}, \bar{S}_{I}$ in the single trace operator. The effective action $\widetilde{S}_{\chi, \eta}$ is given by

$$
\widetilde{S}_{\chi, \eta}=-\sum_{i}\left(\left(\bar{\eta}_{i} \eta_{i}\right)+\left(\bar{\chi}_{i} \chi_{i}\right)\right)-\frac{\lambda}{N} \sum_{i, j} t_{i} t_{j} \underbrace{\frac{n_{i} \cdot \bar{n}_{j}}{\left|x_{i j}\right|}}_{=d_{i j}}\left(\bar{\chi}_{i} \chi_{j}\right)\left(\bar{\eta}_{j} \eta_{i}\right) .
$$

For the next step, we integrate in the"bilocal" fields $\rho_{i j}$ and $\bar{\rho}_{i j}$

$$
G_{m}=\frac{1}{Z_{\rho, \bar{\rho}}} \int d \rho d \bar{\rho} d \eta d \bar{\eta} d \chi d \bar{\chi} \mathcal{O}^{S} \exp \left[-\frac{N}{\lambda} \sum_{\substack{i, j \\ i \neq j}} \rho_{i j} \bar{\rho}_{j i}-\tilde{S}_{\chi, \eta}\right]
$$

where

$$
Z_{\rho, \bar{\rho}}=\int d \rho d \bar{\rho} \exp \left[-\frac{N}{\lambda} \sum_{\substack{i, j \\ i \neq j}} \rho_{i j} \bar{\rho}_{j i}\right]
$$

Shifting the bilocal fields $\rho, \bar{\rho}$ by

$$
\rho_{i j} \mapsto \rho_{i j}-\frac{\lambda}{N} \sqrt{t_{i} t_{j} d_{i j}}\left(\bar{\chi}_{i} \chi_{j}\right), \quad \bar{\rho}_{j i} \mapsto \bar{\rho}_{j i}-\frac{\lambda}{N} \sqrt{t_{i} t_{j} d_{i j}}\left(\bar{\eta}_{j} \eta_{i}\right)
$$

the action of (4.15) can be written as

$$
\begin{aligned}
S_{\rho, \chi, \eta}= & \frac{N}{\lambda} \sum_{\substack{i, j \\
i \neq j}} \rho_{i j} \bar{\rho}_{j i}-\sum_{i, j} \sqrt{t_{i} t_{j} d_{i j}}\left(\rho_{i j}\left(\bar{\eta}_{j} \eta_{i}\right)+\left(\bar{\chi}_{i} \chi_{j}\right) \bar{\rho}_{j i}\right) \\
& -\sum_{i}\left(\left(\bar{\eta}_{i} \eta_{i}\right)+\left(\bar{\chi}_{i} \chi_{i}\right)\right) .
\end{aligned}
$$

Finally we integrate out the fermions and obtain the expression

$$
G_{m}=\frac{1}{Z_{\rho, \bar{\rho}}} \int d \rho d \bar{\rho}\left\langle\mathcal{O}^{S}\right\rangle_{\chi, \eta} \exp \left(N S_{\mathrm{eff}}\right)
$$

Let us explain the definitions of each quantity in the formula: first the effective action is given by

$$
S_{\text {eff }}=-\frac{1}{\lambda} \operatorname{tr}_{m}(\rho \bar{\rho})+\operatorname{tr}_{m} \log \Sigma_{\eta}+\operatorname{tr}_{m} \log \Sigma_{\chi},
$$

where $\operatorname{tr}_{k}$ means a trace of matrices of size $k$. Second $\Sigma_{\eta, \chi}$ are $m \times m$ matrices defined by

$$
\Sigma_{\eta} \equiv \mathbf{1}_{m}+\hat{\rho}, \quad \Sigma_{\chi} \equiv \mathbf{1}_{m}+\hat{\bar{\rho}}
$$

where $\mathbf{1}_{m}$ is the identity matrix of size $m$ and $\hat{\rho}$ and $\hat{\bar{\rho}}$ are defined by

$$
\hat{\rho}_{i j}=\sqrt{t_{i} d_{i j} t_{j}} \rho_{i j}, \quad \hat{\bar{\rho}}_{i j}=\sqrt{t_{j} d_{j i} t_{i}} \bar{\rho}_{i j} .
$$


Third the expectation value $\left\langle\mathcal{O}^{S}\right\rangle_{\chi, \eta}$ is given by

$$
\left\langle\mathcal{O}^{S}\right\rangle_{\chi, \eta}=\frac{\int d \eta d \bar{\eta} d \chi d \bar{\chi} \mathcal{O}^{S} \exp \left(\bar{\eta} \Sigma_{\eta}^{T} \eta+\bar{\chi} \Sigma_{\chi}^{T} \chi\right)}{\int d \eta d \bar{\eta} d \chi d \bar{\chi} \exp \left(\bar{\eta} \Sigma_{\eta}^{T} \eta+\bar{\chi} \Sigma_{\chi}^{T} \chi\right)}
$$

where $\Sigma_{\eta, \chi}^{T}$ means a transposition of the matrix $\Sigma_{\eta, \chi}$.

Let us now explain how to evaluate (4.23) in practice using the following single-trace operator as an example:

$$
\mathcal{O}(y)=\operatorname{tr}\left(Y^{A_{1}} \bar{Y}_{B_{1}} \ldots Y^{A_{L}} \bar{Y}_{B_{L}}\right)(y) .
$$

We first replace the scalar fields in the trace by their classical solutions (4.12)

$$
\mathcal{O}(y) \mapsto \mathcal{O}^{S}(y)=\frac{(-1)^{L}}{k^{2 L}} \sum_{\{i, j\}} \prod_{s=1}^{L} \frac{t_{i_{s}} t_{j_{s}} \bar{n}_{i_{s}}^{A_{s}} n_{j_{s}, B_{s}}}{\left|y-x_{i_{s}}\right|\left|y-x_{j_{s}}\right|} \prod_{s=1}^{L} \chi_{i_{s}} \bar{\eta}_{i_{s}} \eta_{j_{s}} \bar{\chi}_{j_{s}},
$$

where $\sum_{\{i, j\}}$ denotes a summation of $i_{s}$ 's and $j_{s}$ 's from 1 to $m$. Then we take the expectation value

$$
\left\langle\mathcal{O}^{S}\right\rangle_{\chi, \eta}=\frac{(-1)^{L}}{k^{2 L}} \sum_{\{i, j\}} \prod_{s=1}^{L} \frac{t_{i_{s}} t_{j_{s}} \bar{n}_{i_{s}}^{A_{s}} n_{j_{s}, B_{s}}}{\left|y-x_{i_{s}}\right|\left|y-x_{j_{s}}\right|} \times\left\langle\chi_{i_{1}} \bar{\eta}_{i_{1}} \eta_{j_{1}} \bar{\chi}_{j_{1}} \chi_{i_{2}} \bar{\eta}_{i_{2}} \eta_{j_{2}} \bar{\chi}_{j_{2}} \cdots\right\rangle_{\chi, \eta} .
$$

The expectation value of $\chi$ 's and $\eta$ 's can be computed by the Wick contractions, which are given by

$$
\left\langle\bar{\eta}_{i}^{a} \eta_{j, b}\right\rangle=\delta_{b}^{a}\left(\Sigma_{\eta}^{-1}\right)_{i j}, \quad\left\langle\bar{\chi}_{i}^{a} \chi_{j, b}\right\rangle=\delta_{b}^{a}\left(\Sigma_{\chi}^{-1}\right)_{i j},
$$

where $a$ and $b$ are gauge indices while $i$ and $j$ run from 1 to $m$ (= the number of giant gravitons).

Large $N$ limit. To proceed, we consider the large $N$ limit. There are two simplifications in the large $N$ limit. Firstly, the integrations over $\rho$ and $\bar{\rho}$ are dominated by the saddle points of the action $S_{\text {eff }}$ in (4.19). We denote the saddle point solutions by $\rho^{*}$ and $\bar{\rho}^{*}$ and the propagators $(4.27)$ can be evaluated at the saddle point. Secondly, the Wick contraction in (4.26) is dominated by the nearest neighboring contraction (see [29] for more detailed explanation). For instance, if we neglect the prefactors in (4.26), the Wick contraction gives

$$
\begin{aligned}
& \left\langle\chi_{i_{1}} \bar{\eta}_{i_{1}} \eta_{j_{1}} \bar{\chi}_{j_{1}} \chi_{i_{2}} \bar{\eta}_{i_{2}} \eta_{j_{2}} \bar{\chi}_{j_{2}} \ldots\right\rangle \stackrel{N \rightarrow \infty}{=}-\left\langle\bar{\eta}_{i_{1}} \eta_{j_{1}}\right\rangle\left\langle\bar{\chi}_{j_{1}} \chi_{i_{2}}\right\rangle \ldots\left\langle\bar{\eta}_{i_{L}} \eta_{j_{L}}\right\rangle\left\langle\bar{\chi}_{j_{L}} \chi_{i_{1}}\right\rangle \\
& =-N^{2 L} \operatorname{tr}_{m}\left[\Sigma_{\eta}^{-1} \Sigma_{\chi}^{-1} \cdots \Sigma_{\eta}^{-1} \Sigma_{\chi}^{-1}\right] \text {. }
\end{aligned}
$$

Reinstating the prefactors, (4.26) can be written as

$$
\left\langle\mathcal{O}^{S}\right\rangle_{\chi, \eta}=-\operatorname{tr}_{m}\left[T^{A_{1}} \bar{T}_{B_{1}} \cdots T^{A_{L}} \bar{T}_{B_{L}}\right]
$$

where

$$
\begin{aligned}
T^{A} & =-\operatorname{diag}\left(\frac{\lambda t_{1} \bar{n}_{1}^{A}}{\left|y-x_{1}\right|}, \frac{\lambda t_{2} \bar{n}_{2}^{A}}{\left|y-x_{2}\right|} \cdots, \frac{\lambda t_{m} \bar{n}_{m}^{A}}{\left|y-x_{m}\right|}\right) \cdot \Sigma_{\eta}^{-1}, \\
\bar{T}_{B} & =\operatorname{diag}\left(\frac{\lambda t_{1} n_{1, B}}{\left|y-x_{1}\right|}, \frac{\lambda t_{2} n_{2, B}}{\left|y-x_{2}\right|} \cdots, \frac{\lambda t_{m} n_{m, B}}{\left|y-x_{m}\right|}\right) \cdot \Sigma_{\chi}^{-1},
\end{aligned}
$$


are $m \times m$ matrices. Alternatively, we can express this as an overlap between a spin-chain state $\left|A_{1} \bar{B}_{1} \cdots A_{L} \bar{B}_{L}\right\rangle$ and a matrix product state $|\mathrm{MPS}\rangle$ defined by

$$
\left\langle\mathcal{O}^{S}\right\rangle_{\chi, \eta}=\left\langle\mathrm{MPS} \mid A_{1} \bar{B}_{1} \cdots A_{L} \bar{B}_{L}\right\rangle
$$

with

$$
\langle\mathrm{MPS}| \equiv-\sum_{\left\{A_{s}, B_{s}\right\}}\left\langle A_{1} \bar{B}_{1} \cdots A_{L} \bar{B}_{L}\right| \operatorname{tr}_{m}\left[T^{A_{1}} \bar{T}_{B_{1}} \cdots T^{A_{L}} \bar{T}_{B_{L}}\right] .
$$

In deriving these expressions, we implicitly assumed that there is no flat direction around the saddle point. However, this is not always the case as we see below for $m=2$. See section 4.2 for discussions on this point.

Physically the saddle-point value of $e^{N S_{\text {eff }}}$ gives the $m$-point function of giant gravitons while the expectation value $\left\langle\mathcal{O}^{S}\right\rangle_{\chi, \eta}$ gives a ratio of the $(m+1)$-point function and the $m$ point function,

$$
\left\langle\mathcal{O}^{S}\right\rangle_{\chi, \eta}=\frac{\left\langle\left(\prod_{j=1}^{m} \mathcal{D}_{M_{j}}\right) \mathcal{O}(y)\right\rangle}{\left\langle\prod_{j=1}^{m} \mathcal{D}_{M_{j}}\right\rangle} .
$$

\subsection{Two-point function: saddle point and MPS}

To find the solution of saddle point equations, we focus on the case of interest with two giant gravitons, namely we take $m=2$. We furthermore set the charges of the two giant gravitons to be identical $M_{1}=M_{2}=M$ and express the ratio $M / N$ by

$$
\omega \equiv \frac{M}{N} .
$$

Note that $\omega$ takes values from 0 to 1 and the case with $\omega=1$ corresponds to the maximal giant graviton.

Setting $m=2$ in (4.20), we find that the effective action is given by

$$
S_{\text {eff }}=-\frac{1}{\lambda}\left(\rho_{12} \bar{\rho}_{21}+\rho_{21} \bar{\rho}_{12}\right)+\log \left[\left(1-t_{1} t_{2} \sqrt{d_{12} d_{21}} \rho_{12} \rho_{21}\right)\left(1-t_{1} t_{2} \sqrt{d_{12} d_{21}} \bar{\rho}_{12} \bar{\rho}_{21}\right)\right] .
$$

Taking variation with respect to $\rho$ 's and $\bar{\rho}$ 's and imposing $\frac{\delta S_{\text {eff }}}{\delta \rho_{i, j}}=\frac{\delta S_{\text {eff }}}{\delta \bar{\rho}_{i, j}}=0$, we obtain

$$
\rho_{12}^{*} \bar{\rho}_{21}^{*}=\rho_{21}^{*} \bar{\rho}_{12}^{*}=\lambda \frac{t_{1} t_{2} \sqrt{d_{12} d_{21}} \rho_{12}^{*} \rho_{21}^{*}}{t_{1} t_{2} \sqrt{d_{12} d_{21}} \rho_{12}^{*} \rho_{21}^{*}-1}=\lambda \frac{t_{1} t_{2} \sqrt{d_{12} d_{21}} \bar{\rho}_{12}^{*} \bar{\rho}_{21}^{*}}{t_{1} t_{2} \sqrt{d_{12} d_{21}} \bar{\rho}_{12}^{*} \bar{\rho}_{21}^{*}-1} .
$$

Solving these equations, we find ${ }^{12}$

$$
\rho_{i j}^{*}=\bar{\rho}_{i j}^{*}, \quad \rho_{12}^{*} \rho_{21}^{*}=\bar{\rho}_{12}^{*} \bar{\rho}_{21}^{*}=\lambda+\frac{1}{t_{1} t_{2} \sqrt{d_{12} d_{21}}} .
$$

\footnotetext{
${ }^{12}$ In fact there is another solution to the saddle point equations,

$$
\rho_{i j}^{*}=-\bar{\rho}_{i j}^{*}, \quad \rho_{12}^{*} \rho_{21}^{*}=\bar{\rho}_{12}^{*} \bar{\rho}_{21}^{*}=-\lambda+\frac{1}{t_{1} t_{2} \sqrt{d_{12} d_{21}}} .
$$

However it will eventually give the same saddle point action and the same MPS (after the integration over $\theta$ which will be defined soon), and therefore do not modify our result for the ratio of correlators. Then we will focus only on the first solution in the rest of the paper.
} 
The effective action at this saddle point is given by

$$
S_{\mathrm{eff}}^{*}=-2-\frac{2}{\lambda t_{1} t_{2} \sqrt{d_{12} d_{21}}}+\log \left[\lambda^{2} t_{1}^{2} t_{2}^{2} d_{12} d_{21}\right] .
$$

To compute the correlation function of giant gravitons with fixed charges, we need to perform integrals of $t$ 's (4.3). Since $M$ is $\mathcal{O}(N)$, some of those integrals can be evaluated using the saddle-point approximation. Solving the saddle-point equations

$$
2 \omega \frac{\partial \log t_{1,2}}{\partial t_{1,2}}-\frac{\partial S_{\mathrm{eff}}^{*}}{\partial t_{1,2}}=0
$$

we get

$$
t_{1}^{*} t_{2}^{*}=\frac{1}{\lambda(\omega-1) \sqrt{d_{12} d_{21}}} .
$$

As is clear from this expression, the saddle-point equation only determines the product $t_{1} t_{2}$. Therefore, we still need to perform an integral of the ratio

$$
e^{i \theta} \equiv \sqrt{\frac{t_{1}}{t_{2}}} .
$$

as we see below.

MPS for two giant gravitons. Let us place the single trace operator at $y=x_{3}$,

$$
\mathcal{O}(y)=\operatorname{tr}\left(Y^{A_{1}} \bar{Y}_{B_{1}} \ldots Y^{A_{L}} \bar{Y}_{B_{L}}\right)\left(x_{3}\right)
$$

and compute the three-point function of two giant gravitons and $\mathcal{O}$.

Evaluating the matrices $T^{A}$ and $\bar{T}_{B}$ at the saddle point (4.36) and (4.40), we get

$$
\begin{aligned}
& T^{A}=-\lambda \operatorname{diag}\left(\frac{t_{1}^{*} \bar{n}_{1}^{A}}{\left|x_{13}\right|} \frac{t_{2}^{*} \bar{n}_{2}^{A}}{\left|x_{23}\right|}\right) \cdot\left(\begin{array}{cc}
1-\omega & -i \rho_{12}^{*} \sqrt{\frac{1-\omega}{\lambda}}\left(\frac{d_{12}}{d_{21}}\right)^{\frac{1}{4}} \\
-i \rho_{21}^{*} \sqrt{\frac{1-\omega}{\lambda}}\left(\frac{d_{21}}{d_{12}}\right)^{\frac{1}{4}} & 1-\omega
\end{array}\right), \\
& \bar{T}_{B}=\lambda \operatorname{diag}\left(\frac{t_{1}^{*} n_{1, B}}{\left|x_{13}\right|}, \frac{t_{2}^{*} n_{2, B}}{\left|x_{23}\right|}\right) \cdot\left(\begin{array}{cc}
1-\omega & -i \rho_{12}^{*} \sqrt{\frac{1-\omega}{\lambda}}\left(\frac{d_{21}}{d_{12}}\right)^{\frac{1}{4}} \\
-i \rho_{21}^{*} \sqrt{\frac{1-\omega}{\lambda}}\left(\frac{d_{12}}{d_{21}}\right)^{\frac{1}{4}} & 1-\omega
\end{array}\right) .
\end{aligned}
$$

To proceed, we make use of the fact that the MPS (4.32) is invariant under the transformation $T^{A} \mapsto U T^{A} U^{-1}$ and $\bar{T}_{B} \mapsto U \bar{T}_{B} U^{-1}$. By choosing an appropriate $U$, we can replace $\rho_{12}^{*}$ and $\rho_{21}^{*}$ in $(4.43)$ with $\sqrt{\rho_{12}^{*} \rho_{21}^{*}}$. As a result, we obtain the following matrices

$$
\begin{aligned}
T_{\theta}^{A} & =\frac{\sqrt{\lambda}}{\left(d_{12} d_{21}\right)^{\frac{1}{4}}}\left(\begin{array}{cc}
-\frac{\bar{n}_{1}^{A} e^{i \theta}}{\left|x_{13}\right|} \sqrt{1-\omega} & i \frac{\bar{n}_{1}^{A} e^{i \theta}}{\left|x_{13}\right|} \sqrt{\omega}\left(\frac{d_{12}}{d_{21}}\right)^{\frac{1}{4}} \\
i \frac{\bar{n}_{2}^{A} e^{-i \theta}}{\left|x_{23}\right|} \sqrt{\omega}\left(\frac{d_{21}}{d_{12}}\right)^{\frac{1}{4}} & -\frac{\bar{n}_{2}^{A} e^{-i \theta}}{\left|x_{23}\right|} \sqrt{1-\omega}
\end{array}\right), \\
\bar{T}_{B, \theta} & =\frac{\sqrt{\lambda}}{\left(d_{12} d_{21}\right)^{\frac{1}{4}}}\left(\begin{array}{cc}
\frac{n_{1, B} e^{i \theta}}{\left|x_{13}\right|} \sqrt{1-\omega} & -i \frac{n_{1, B} e^{i \theta}}{\left|x_{13}\right|} \sqrt{\omega}\left(\frac{d_{21}}{d_{12}}\right)^{\frac{1}{4}} \\
-i \frac{n_{2, B} e^{-i \theta}}{\left|x_{23}\right|} \sqrt{\omega}\left(\frac{d_{12}}{d_{21}}\right)^{\frac{1}{4}} & \frac{n_{2, B} e^{-i \theta}}{\left|x_{23}\right|} \sqrt{1-\omega}
\end{array}\right) .
\end{aligned}
$$


Here $e^{i \theta}$ is the ratio of $t$ 's defined in (4.41). As is clear from this expression, the MPS depens on $\theta$ which parametrizes the flat direction around the saddle point. Therefore, the correct result would be given by an integral over this direction;

$$
\left\langle\mathcal{O}^{S}\right\rangle_{\chi, \eta}=\int_{0}^{2 \pi} \frac{d \theta}{2 \pi}\left\langle\operatorname{MPS}_{\theta} \mid A_{1} \bar{B}_{1} \cdots A_{L} \bar{B}_{L}\right\rangle
$$

with

$$
\left\langle\mathrm{MPS}_{\theta}\right| \equiv-\sum_{\left\{A_{s}, B_{s}\right\}}\left\langle A_{1} \bar{B}_{1} \cdots A_{L} \bar{B}_{L}\right| \operatorname{tr}_{m}\left[T_{\theta}^{A_{1}} \bar{T}_{B_{1}, \theta} \cdots T_{\theta}^{A_{L}} \bar{T}_{B_{L}, \theta}\right]
$$

\subsection{Structure constants of BPS single-trace operators}

Let us now use the results above to derive an expression for the structure constant of the single-trace operator.

Integral representation. We first consider the BPS single-trace operator, given by

$$
\mathcal{O}_{L}^{\circ}\left(x_{3}\right)=\operatorname{tr}\left[\left(n_{3} \cdot Y \bar{n}_{3} \cdot \bar{Y}\right)^{L}\right]\left(x_{3}\right) .
$$

Using the results above, we find

$$
\frac{\left\langle\mathcal{D}_{M} \mathcal{D}_{M} \mathcal{O}_{L}^{\circ}\left(x_{3}\right)\right\rangle}{\left\langle\mathcal{D}_{M} \mathcal{D}_{M}\right\rangle}=\int_{0}^{2 \pi} \frac{d \theta}{2 \pi} \operatorname{tr}_{2}\left[\left(\mathcal{T}_{\theta} \overline{\mathcal{T}}_{\theta}\right)^{L}\right],
$$

with

$$
\begin{aligned}
& \mathcal{T}_{\theta} \equiv \sum_{A} n_{3, A} T_{\theta}^{A}=\frac{\sqrt{\lambda}}{\left(d_{12} d_{21}\right)^{\frac{1}{4}}}\left(\begin{array}{cc}
-d_{31} e^{i \theta} \sqrt{1-\omega} & i d_{31} e^{i \theta} \sqrt{\omega}\left(\frac{d_{12}}{d_{21}}\right)^{\frac{1}{4}} \\
i d_{32} e^{-i \theta} \sqrt{\omega}\left(\frac{d_{21}}{d_{12}}\right)^{\frac{1}{4}} & -d_{32} e^{-i \theta} \sqrt{1-\omega}
\end{array}\right) \\
& \overline{\mathcal{T}}_{\theta} \equiv \sum_{B} \bar{n}_{3}^{B} \bar{T}_{\theta, B}=\frac{\sqrt{\lambda}}{\left(d_{12} d_{21}\right)^{\frac{1}{4}}}\left(\begin{array}{cc}
d_{13} e^{i \theta} \sqrt{1-\omega} & -i d_{13} e^{i \theta} \sqrt{\omega}\left(\frac{d_{21}}{d_{12}}\right)^{\frac{1}{4}} \\
-i d_{23} e^{-i \theta} \sqrt{\omega}\left(\frac{d_{12}}{d_{21}}\right)^{\frac{1}{4}} & d_{23} e^{-i \theta} \sqrt{1-\omega}
\end{array}\right) .
\end{aligned}
$$

To evaluate (4.48) it is convenient to consider a generating function of structure constants,

$$
\sum_{L=0}^{\infty} s \frac{\left\langle\mathcal{D}_{M} \mathcal{D}_{M} \mathcal{O}_{L}^{\circ}\left(x_{3}\right)\right\rangle}{\left\langle\mathcal{D}_{M} \mathcal{D}_{M}\right\rangle}=-\int_{0}^{2 \pi} \frac{d \theta}{2 \pi} \operatorname{tr}_{2}\left[\frac{1}{1-s \mathcal{T}_{\theta} \overline{\mathcal{T}_{\theta}}}\right]
$$

To proceed, we evaluate the right hand side by diagonalizing the matrices $\mathcal{T}_{\theta} \overline{\mathcal{T}}_{\theta}$, perform the integral, and read off the coefficient in front of $s^{L} .{ }^{13}$ The result reads

$$
\frac{\left\langle\mathcal{D}_{M} \mathcal{D}_{M} \mathcal{O}_{L}^{\circ}\left(x_{3}\right)\right\rangle}{\left\langle\mathcal{D}_{M} \mathcal{D}_{M}\right\rangle}=-\lambda^{L}\left(\frac{d_{23} d_{32} d_{31} d_{13}}{d_{12} d_{21}}\right)^{\frac{L}{2}} \xi^{-\frac{L}{2}} \oint \frac{d s}{2 \pi i s^{1+L}} \frac{1-\xi s^{2}}{\sqrt{\mathcal{P}(s)}},
$$

where $\mathcal{P}(s)$ is given by

$$
\mathcal{P}(s) \equiv 1-2 \omega(1+\xi) s+\left[-2 \xi+8 \xi \omega+\omega^{2}(1-\xi)^{2}\right] s^{2}-2 \omega \xi(1+\xi) s^{3}+\xi^{2} s^{4} .
$$

\footnotetext{
${ }^{13}$ Here it is important to keep $s$ being small to make the power series convergent in (4.50). We will give the details of the computations in appendix B.
} 
Dividing this by the normalization factor $\mathcal{N}_{\mathcal{O}_{L}^{\circ}}$ and stripping off the kinematic factors, we arrive at the following expression for the structure constants;

$$
\sum_{p=-\frac{L}{2}}^{\frac{L}{2}} \xi^{p} D_{M \mid L}^{(p)}=-\frac{\xi^{-\frac{L}{2}}}{\sqrt{L}} \oint \frac{d s}{2 \pi i s^{1+L}} \frac{1-\xi s^{2}}{\sqrt{\mathcal{P}(s)}} .
$$

For a given $L$, the integral (4.53) can be readily evaluated. For instance, for small values of $L$, we obtain

$$
\begin{aligned}
& L=1: \quad \sum_{p=-\frac{1}{2}}^{\frac{1}{2}} \xi^{p} D_{M \mid 1}^{(p)}=-\omega\left(\xi^{-\frac{1}{2}}+\xi^{\frac{1}{2}}\right), \\
& L=2: \quad \sum_{p=-1}^{1} \xi^{p} D_{M \mid 2}^{(p)}=-\frac{\omega^{2}}{\sqrt{2}}\left(\xi^{-1}+4\left(1-\omega^{-1}\right)+\xi\right), \\
& L=3: \quad \sum_{p=-\frac{3}{2}}^{\frac{3}{2}} \xi^{p} D_{M \mid 3}^{(p)}=-\frac{\omega^{3}}{\sqrt{3}}\left[\xi^{-\frac{3}{2}}+\frac{3(1-\omega)(1-3 \omega)}{\omega^{2}}\left(\xi^{-\frac{1}{2}}+\xi^{\frac{1}{2}}\right)+\xi^{\frac{3}{2}}\right] .
\end{aligned}
$$

Closed-form expressions for special cases. It is however difficult to write down a closed-form expression for general $L, \xi$ and $\omega$. The exceptions are for the maximal giant graviton $(\omega=1)$ and for the twisted-translated kinematics $(\xi=-1)$. The former is given by

$$
\sum_{p=-\frac{L}{2}}^{\frac{L}{2}} \xi^{p} D_{N \mid L}^{(p)}=-\frac{1}{\sqrt{L}}\left(\xi^{-\frac{L}{2}}+\xi^{\frac{L}{2}}\right)
$$

which is equivalent to

$$
D_{N \mid L}^{(p)}=-\frac{1}{\sqrt{L}}\left(\delta_{p, \frac{L}{2}}+\delta_{p,-\frac{L}{2}}\right)
$$

On the other hand, in the twisted-translated kinematics $(\xi=-1)$, we have

$$
\begin{aligned}
\mathfrak{D}_{M \mid L} & =\sum_{p=-\frac{L}{2}}^{\frac{L}{2}}(-1)^{p} D_{M \mid L}^{(p)} \\
& = \begin{cases}-\frac{(-1)^{\frac{L}{2}}}{\sqrt{L}}\left[P_{\frac{L}{2}}\left(-1+4 \omega-2 \omega^{2}\right)+P_{\frac{L}{2}-1}\left(-1+4 \omega-2 \omega^{2}\right)\right] & L: \text { even } \\
0 & L: \text { odd }\end{cases}
\end{aligned}
$$

where $P_{\frac{L}{2}}$ is the Legendre polynomial.

\subsection{Structure constants of non-BPS single-trace operators}

We next study the structure constant of the non-BPS single-trace operator. As mentioned in section 2, in this paper we focus on the twisted-translated frame, which amounts to setting

$$
n_{1,2}=\left(1,0,0, \kappa a_{1,2}\right), \quad \bar{n}_{1,2}=\left(-\kappa a_{1,2}, 0,0,1\right), \quad x_{1,2}=\left(0, a_{1,2}, 0\right) .
$$


Result for the structure constant. To further simplify the analysis, we consider a symmetric configuration in which the single-trace operator is at the origin and the giant gravitons are placed symmetrically around it; namely we set $a_{1}=-a_{2}=+1$ and $a_{3}=0$ in (2.42). Then the matrices that enter in the MPS representation, (4.44), simplify to the following,

$$
\begin{aligned}
T_{\theta}^{1} & =\sqrt{\kappa \lambda} e^{-i \frac{\pi}{4}}\left(\begin{array}{cc}
e^{i \theta} \sqrt{1-\omega} & -i e^{i\left(\theta-\frac{\pi}{4}\right)} \sqrt{\omega} \\
i e^{-i\left(\theta-\frac{\pi}{4}\right)} \sqrt{\omega} & -e^{-i \theta} \sqrt{1-\omega}
\end{array}\right), \\
\bar{T}_{4, \theta} & =\sqrt{\kappa \lambda} e^{-i \frac{\pi}{4}}\left(\begin{array}{cc}
e^{i \theta} \sqrt{1-\omega} & -i e^{i\left(\theta+\frac{\pi}{4}\right)} \sqrt{\omega} \\
i e^{-i\left(\theta+\frac{\pi}{4}\right)} \sqrt{\omega} & -e^{-i \theta} \sqrt{1-\omega}
\end{array}\right), \\
T_{\theta}^{4} & =\sqrt{\frac{\lambda}{\kappa}} e^{-i \frac{\pi}{4}}\left(\begin{array}{cc}
-e^{i \theta} \sqrt{1-\omega} & i e^{i\left(\theta-\frac{\pi}{4}\right)} \sqrt{\omega} \\
i e^{-i\left(\theta-\frac{\pi}{4}\right)} \sqrt{\omega} & -e^{-i \theta} \sqrt{1-\omega}
\end{array}\right), \\
\bar{T}_{1, \theta} & =\sqrt{\frac{\lambda}{\kappa}} e^{-i \frac{\pi}{4}}\left(\begin{array}{cc}
e^{i \theta} \sqrt{1-\omega} & -i e^{i\left(\theta+\frac{\pi}{4}\right)} \sqrt{\omega} \\
-i e^{-i\left(\theta+\frac{\pi}{4}\right)} \sqrt{\omega} & e^{-i \theta} \sqrt{1-\omega}
\end{array}\right),
\end{aligned}
$$

and all the other $T_{\theta}^{A}$ 's and $\bar{T}_{B, \theta}$ are zero.

To compute the structure constant $\mathfrak{D}_{M \mid \mathcal{O}}$, we then compute the matrix trace in (4.46), perform the $\theta$ integral in (4.45) and divide the result by the normalization $\mathcal{N}_{\mathcal{O}}$. As discussed in [80], $\mathcal{N}_{\mathcal{O}}$ can be expressed in terms of the norm of the spin-chain state $\langle\mathcal{O} \mid \mathcal{O}\rangle$ as follows

$$
\mathcal{N}_{\mathcal{O}}=L \lambda^{2 L}\langle\mathcal{O} \mid \mathcal{O}\rangle
$$

where the prefactor $L$ is the number of different Wick contractions related by cyclic permutations (see [80] for details) and $\lambda^{2 L}$ comes from the normalization of propagators. Finally, factoring out the kinematic factor in (2.42), we arrive at the expression,

$$
\mathfrak{D}_{M \mid \mathcal{O}}=\frac{(-1)^{J+1}}{2^{\Delta-J} \sqrt{L\langle\mathcal{O} \mid \mathcal{O}\rangle}} \int_{0}^{2 \pi} \frac{d \theta}{2 \pi}\left\langle B_{\theta} \mid \mathcal{O}\right\rangle
$$

where the matrix product state $\left\langle B_{\theta}\right|$ is defined by

$$
\left\langle B_{\theta}\right| \equiv \sum_{A_{s}, B_{s}=1,4}\left\langle A_{1} \bar{B}_{1} \cdots A_{L} \bar{B}_{L}\right| \operatorname{tr}_{2}\left[\mathrm{t}^{A_{1}} \overline{\mathrm{t}}_{B_{1}} \cdots \mathrm{t}^{\left.A_{L} \overline{\mathrm{t}}_{B_{L}}\right]}\right.
$$

with $^{14}$

$$
\begin{array}{rlr}
\mathrm{t}^{1}=\left(\begin{array}{cc}
e^{i \theta} \sqrt{1-\omega} & -i \sqrt{\omega} \\
i \sqrt{\omega} & -e^{-i \theta} \sqrt{1-\omega}
\end{array}\right), & \overline{\mathrm{t}}_{4}=\left(\begin{array}{cc}
e^{i \theta} \sqrt{1-\omega} & \sqrt{\omega} \\
\sqrt{\omega} & -e^{-i \theta} \sqrt{1-\omega}
\end{array}\right), \\
\mathrm{t}^{4}=\left(\begin{array}{cc}
-e^{i \theta} \sqrt{1-\omega} & i \sqrt{\omega} \\
i \sqrt{\omega} & -e^{-i \theta} \sqrt{1-\omega}
\end{array}\right), & \overline{\mathrm{t}}_{1}=\left(\begin{array}{cc}
e^{i \theta} \sqrt{1-\omega} & \sqrt{\omega} \\
-\sqrt{\omega} & e^{-i \theta} \sqrt{1-\omega}
\end{array}\right) .
\end{array}
$$

\footnotetext{
${ }^{14}$ Recall that $\omega$ is related to the charge of the giant graviton $M$ by $\omega \equiv M / N$.
} 
Simplification for the maximal giant graviton. For the maximal giant gravitons $\omega=1$, the matrices in (4.63) all become off-diagonal, and the products of $t$ and $\bar{t}$ take the following simple form;

$$
\begin{aligned}
\mathrm{t}_{1} \overline{\mathrm{t}}_{4}=\left(\begin{array}{cc}
-i & 0 \\
0 & i
\end{array}\right), & \mathrm{t}_{1} \overline{\mathrm{t}}_{1}=\left(\begin{array}{cc}
i & 0 \\
0 & i
\end{array}\right), \\
\mathrm{t}_{4} \overline{\mathrm{t}}_{4}=\left(\begin{array}{cc}
i & 0 \\
0 & i
\end{array}\right), & \mathrm{t}_{1} \overline{\mathrm{t}}_{1}=\left(\begin{array}{cc}
-i & 0 \\
0 & i
\end{array}\right) .
\end{aligned}
$$

Since these matrices do not depend on $\theta$, the integral of $\theta$ can be trivially performed. As a result, we can replace (4.61) with the following expression

$$
\mathfrak{D}_{N \mid \mathcal{O}}=-\frac{(-i)^{J}}{2^{\Delta-J}} \frac{\langle\mathcal{B} \mid \mathcal{O}\rangle}{\sqrt{L\langle\mathcal{O} \mid \mathcal{O}\rangle}}
$$

where the state $\langle\mathcal{B}|$ is defined by

$$
\langle\mathcal{B}| \equiv \sum_{A_{s}, B_{s}=1,4}\left\langle A_{1} \bar{B}_{1} \cdots A_{L} \bar{B}_{L}\right|\left(1+(-1)^{J}\right) .
$$

Here $J$ is the $\mathrm{U}(1)$ R-charge, which counts

$$
J=L-(\text { number of } 4 \text { on odd sites })-(\text { number of } \overline{1} \text { on even sites }) \text {. }
$$

We can also check that the result (4.65) matches the one computed from a different approach explained in appendix A.

In the rest of this paper, we use (4.61) and (4.65) to evaluate the structure constants of non-BPS single-trace operators. For this purpose, we need to compute two quantities in the spin chain; the overlap $\langle B \mid \mathcal{O}\rangle$ and the norm $\langle\mathcal{O} \mid \mathcal{O}\rangle$. In the next section, we present conjectures for these observables based on the coordinate Bethe ansatz we developed in section 3 .

\section{Main results}

In this section, we use the coordinate Bethe ansatz in section 3 and evaluate the overlap $\langle B \mid \mathcal{O}\rangle$ and the norm $\langle\mathcal{O} \mid \mathcal{O}\rangle$. For reader's convenience, we first present a summary of results and later explain the details. The numerical data used for checking our formula, such as the solutions to the Bethe equations and the results for the overlap, is summarized in appendix C.

In what follows, we label a Bethe state in terms of its Bethe roots; namely we express $|\mathcal{O}\rangle$ as $|\mathbf{u}, \mathbf{w}, \mathbf{v}\rangle$ where $\mathbf{u}, \mathbf{w}$ and $\mathbf{v}$ are sets of Bethe roots for each Dynkin node (see figure 1).

\subsection{Summary of results}

Selection rules for the overlap. As a result of the numerical experiments, we found a set of selection rules in order for the overlap to be non-zero. The selection rules apply both to the maximal giant graviton $\langle\mathcal{B} \mid \mathcal{O}\rangle$ and the non-maximal giant gravitons $\int \frac{d \theta}{2 \pi}\left\langle B_{\theta} \mid \mathcal{O}\right\rangle$. 
The first and the most obvious selection rule is

0. The numbers of Bethe roots for each node need to satisfy

$$
K_{\mathbf{u}}=K_{\mathbf{v}}=K_{\mathbf{w}}
$$

This simply follows from the fact that $\left\langle B_{\theta}\right|$ only contains fields $Y^{1}, Y^{4}, \bar{Y}_{1}$ and $\bar{Y}_{4}$ : in order for $|\mathbf{u}, \mathbf{w}, \mathbf{v}\rangle$ to contain kets involving $Y^{1}, Y^{4}, \bar{Y}_{1}, \bar{Y}_{4}$ only, we need to set $K_{\mathbf{u}}=K_{\mathbf{v}}=K_{\mathbf{w}}$. Note that this is just a consequence of the global symmetry, not the integrability of the matrix product state.

In addition to this constraint, the overlap also obeys the following "parity conditions":

1. The U(1) R-charge of the state, $J=L-\frac{K_{\mathbf{u}}+K_{\mathbf{v}}}{2}$, must be even.

2. The rapidities of the right node must be $(-1)$ times the rapidities of the left node:

$$
\mathbf{v}=-\mathbf{u}
$$

3. The rapidities of the middle node should be parity-symmetric, namely

$$
\mathbf{w}= \begin{cases}\left(w_{1},-w_{1}, w_{2},-w_{2}, \ldots\right) & K_{\mathbf{w}}: \text { even } \\ \left(w_{1},-w_{1}, w_{2},-w_{2}, \ldots, 0\right) & K_{\mathbf{w}}: \text { odd }\end{cases}
$$

These selection rules are almost identical to the selection rules found for the structure constant of two giant gravitons and a single-trace operator in the $\mathrm{SO}(6)$ sector of $\mathcal{N}=4$ SYM $[29,30]$. The only difference is the roles of the rapidities: in ABJM, the left and the right nodes are momentum carrying nodes and the middle node is auxiliary while in $\mathcal{N}=4$ SYM, the middle node is momentum carrying and the left and right nodes are auxiliary.

As discussed in $[29,43,46,104]$, the existence of these selection rules indicate that the matrix product states $\langle\mathcal{B}|$ and $\int \frac{d \theta}{2 \pi}\left\langle B_{\theta}\right|$ are integrable boundary states. In particular, we want to emphasize that the non-maximal giant gravitons also satisfy the selection rule, implying that they correspond to integrable boundary states as well.

Determinant formula for the maximal giant graviton. For the maximal giant graviton $(\omega=1)$, we found a closed-form expression for the structure constant. Before writing down the result, let us first clarify our convention and ordering of the rapidities satisfying the selection rules;

$$
\begin{aligned}
& \mathbf{u}=\left(u_{1}, u_{2}, \ldots, u_{K_{\mathbf{u}}}\right), \\
& \mathbf{v}=-\mathbf{u}=\left(-u_{1},-u_{2}, \ldots,-u_{K_{\mathbf{u}}}\right), \\
& \mathbf{w}=\left\{\begin{array}{ll}
\left(w_{1},-w_{1}, w_{2},-w_{2}, \ldots, w_{\frac{K_{\mathbf{w}}}{2}},-w_{\frac{K_{\mathbf{w}}}{2}}\right) & K_{\mathbf{w}}: \text { even } \\
\left(w_{1},-w_{1}, w_{2},-w_{2}, \ldots, w_{\left\lceil\frac{K_{\mathbf{w}}}{2}\right\rceil},-w_{\left\lceil\frac{K_{\mathbf{w}}}{2}\right\rceil}, 0\right) & K_{\mathbf{w}}: \text { odd }
\end{array},\right.
\end{aligned}
$$

where $\left\lceil\frac{K_{\mathrm{w}}}{2}\right\rceil$ means the largest integer not larger than $\frac{K_{\mathrm{w}}}{2}$. 
The result for the normalized overlap reads

$$
\frac{\langle\mathcal{B} \mid \mathbf{u}, \mathbf{w},-\mathbf{u}\rangle}{\sqrt{\langle\mathbf{u}, \mathbf{w},-\mathbf{u} \mid \mathbf{u}, \mathbf{w},-\mathbf{u}\rangle}}=-2 \sqrt{\prod_{j=1}^{K_{\mathbf{u}}}\left(u_{j}^{2}+\frac{1}{4}\right) \prod_{k=1}^{\left\lceil\frac{K_{\mathbf{w}}}{2}\right\rceil} \frac{1}{w_{k}^{2}\left(w_{k}^{2}+\frac{1}{4}\right)} \frac{\operatorname{det} G_{+}}{\operatorname{det} G_{-}} .}
$$

Here $\operatorname{det} G_{ \pm}$are the Gaudin-like determinants which we will explain in the next subsection. The result leads to the following expression for the structure constant:

$$
\mathfrak{D}_{N \mid \mathcal{O}}=\frac{i^{J}+(-i)^{J}}{2^{\Delta-J} \sqrt{L}} \sqrt{\prod_{j=1}^{K_{\mathrm{u}}}\left(u_{j}^{2}+\frac{1}{4}\right) \prod_{k=1}^{\left\lceil\frac{K_{\mathrm{w}}}{2}\right\rceil} \frac{1}{w_{k}^{2}\left(w_{k}^{2}+\frac{1}{4}\right)} \frac{\operatorname{det} G_{+}}{\operatorname{det} G_{-}} .}
$$

In (5.5) and (5.6), we neglected an overall phase factor which is ambiguous (see the discussion in section 2.2). These are the main results of this paper. Below we explain the details of the formula and the derivation.

For the non-maximal giant gravitons, we have not been able to find a closed-form expression analogous to (5.6). The results for a sample of Bethe states are summarized in appendix C. We leave it for future investigations to find a determinant formula for the non-maximal giant gravitons.

\subsection{Norms and Gaudin determinant}

Norms for the coordinate Bethe states. The norms of the Bethe states are often given by the so-called Gaudin determinant, which are given by the logarithmic derivative of the Bethe equations. In the case at hand, the relevant Gaudin matrix is given by

$$
G=\left(\begin{array}{ccc}
\partial_{u_{i}} \phi_{u_{j}} & \partial_{u_{i}} \phi_{w_{j}} & \partial_{u_{i}} \phi_{v_{j}} \\
\partial_{w_{i}} \phi_{u_{j}} & \partial_{w_{i}} \phi_{w_{j}} & \partial_{w_{i}} \phi_{v_{j}} \\
\partial_{v_{i}} \phi_{u_{j}} & \partial_{v_{i}} \phi_{w_{j}} & \partial_{v_{i}} \phi_{v_{j}}
\end{array}\right) \text {. }
$$

where $\phi$ 's was defined through the Bethe ansatz equations (3.7).

We conjecture that the norms of the Bethe states in the SU(4)-invariant alternating spin chain are given by

$$
\begin{aligned}
\langle\mathbf{u}, \mathbf{w}, \mathbf{v} \mid \mathbf{u}, \mathbf{w}, \mathbf{v}\rangle= & \left(\prod_{i<j} \frac{S\left(u_{i}, u_{j}\right)}{S\left(u_{i}^{*}, u_{j}^{*}\right)}\right)^{\frac{1}{2}}\left(\prod_{i<j} \frac{S\left(v_{i}, v_{j}\right)}{S\left(v_{i}^{*}, v_{j}^{*}\right)}\right)^{\frac{1}{2}}\left(\prod_{i<j} \frac{S\left(w_{i}, w_{j}\right)}{S\left(w_{i}^{*}, w_{j}^{*}\right)}\right)^{\frac{1}{2}} \\
& \times\left(\prod_{j} \frac{1}{\partial_{u} p\left(u_{j}\right)}\right)\left(\prod_{k} \frac{1}{\partial_{v} p\left(v_{k}\right)}\right) \operatorname{det} G,
\end{aligned}
$$

where the states are normalized using the coordinate Bethe ansatz described in section 3 and $\langle\mathbf{u}, \mathbf{w}, \mathbf{v}| \equiv[|\mathbf{u}, \mathbf{w}, \mathbf{v}\rangle]^{\dagger} . S(u, v)$ is the S-matrix

$$
S(u, v) \equiv \frac{u-v-i}{u-v+i}
$$

while $p(u)$ is the momentum of a magnon with rapidity $u$

$$
p(u) \equiv \frac{1}{i} \log \frac{u+\frac{i}{2}}{u-\frac{i}{2}} \quad \Rightarrow \quad \partial_{u} p(u)=\frac{-1}{u^{2}+\frac{1}{4}} .
$$


The conjecture (5.8) passes several nontrivial tests: it correctly reproduces the results for the $\mathrm{SU}(2) \times \mathrm{SU}(2)$ sector (see for instance [105]) and it is satisfied by all the Bethe states listed in appendix C.

Simplifications for the parity-symmetric states. For the states satisfying the parity conditions $|\mathbf{u}, \mathbf{w},-\mathbf{u}\rangle$, the formula for the norm simplifies further.

First the prefactor in (5.8) simplifies to

$$
\langle\mathbf{u}, \mathbf{w},-\mathbf{u} \mid \mathbf{u}, \mathbf{w},-\mathbf{u}\rangle=\prod_{k=1}^{\left\lceil\frac{K_{\mathbf{w}}}{2}\right\rceil} \frac{\left(w_{k}-\frac{i}{2}\right)\left(w_{k}^{*}+\frac{i}{2}\right)}{\left(w_{k}+\frac{i}{2}\right)\left(w_{k}^{*}-\frac{i}{2}\right)} \prod_{j=1}^{K_{\mathbf{u}}}\left(u_{j}^{2}+\frac{1}{4}\right)^{2} \operatorname{det} G .
$$

Note that we are using the convention and the ordering given in (5.4).

Second the Gaudin determinant for the parity-symmetric states factorizes into a determinants of submatrices. This factorization was already observed in $\mathcal{N}=4 \mathrm{SYM}$, and we will show below that a similar factorization holds also for ABJM. Since the derivations for the even $K_{\mathrm{w}}$ cases is similar to the one for the odd $K_{\mathrm{w}}$ cases, we first discuss the odd $K_{\mathrm{w}}$ cases.

For this purpose, we divide the rapidities $\mathbf{w}=\left(w_{1},-w_{1}, w_{2},-w_{2}, \cdots, 0\right)$ into three parts, $\mathbf{w}^{(+)}=\left(w_{1}, w_{2}, \cdots\right)$ denotes the "postive" part of Bethe roots, $\mathbf{w}^{(-)}=$ $\left(-w_{1},-w_{2}, \cdots\right)$ denotes the "negative" part, and $\mathbf{w}^{(0)}=(0)$. As the first step, We reorder the rows and columns of Gaudin determinant to express it as

$$
\operatorname{det} G=\operatorname{det}\left(\begin{array}{ccccc}
U_{u} & U_{+} & U_{0} & U_{v} & U_{-} \\
W_{+u} & W_{++} & W_{+0} & W_{+v} & W_{+-} \\
W_{0 u} & W_{0+} & W_{00} & W_{0 v} & W_{0-} \\
V_{u} & V_{+} & V_{0} & V_{v} & V_{-} \\
W_{-u} & W_{-+} & W_{-0} & W_{-v} & W_{--}
\end{array}\right)
$$

with

$$
\begin{aligned}
& {\left[U_{u}\right]_{i j} \equiv \partial_{u_{i}} \phi_{u_{j}}, \quad\left[U_{ \pm}\right]_{i j} \equiv \partial_{u_{i}} \phi_{w \pm_{j}}, \quad\left[U_{0}\right]_{i j} \equiv \partial_{u_{i}} \phi_{w^{(0)}}, \quad\left[U_{v}\right]_{i j} \equiv \partial_{u_{i}} \phi_{v_{j}},} \\
& {\left[W_{ \pm u}\right]_{i j} \equiv \partial_{w_{i}^{( \pm)}} \phi_{u_{j}}, \quad\left[W_{ \pm \pm}\right]_{i j} \equiv \partial_{w_{i}^{( \pm)}} \phi_{w_{j}^{( \pm)}}, \quad\left[W_{ \pm 0}\right]_{i j} \equiv \partial_{w_{i}^{( \pm)}} \phi_{w^{(0)}},} \\
& {\left[W_{ \pm \mp}\right]_{i j} \equiv \partial_{w_{i}^{( \pm)}} \phi_{w_{j}^{(\mp)}}, \quad\left[W_{ \pm v}\right]_{i j} \equiv \partial_{w_{i}^{( \pm)}} \phi_{v_{j}}, \quad\left[W_{0 u}\right]_{i j} \equiv \partial_{w^{(0)}} \phi_{u_{j}},} \\
& {\left[W_{0 \pm}\right]_{i j} \equiv \partial_{w^{(0)}} \phi_{w_{j}^{( \pm)}}, \quad\left[W_{00}\right]_{i j} \equiv \partial_{w^{(0)}} \phi_{w^{(0)}}, \quad\left[W_{0 v}\right]_{i j} \equiv \partial_{w 0} \phi_{v_{j}},} \\
& {\left[V_{u}\right]_{i j} \equiv \partial_{v_{i}} \phi_{u_{j}}, \quad\left[V_{ \pm}\right]_{i j} \equiv \partial_{v_{i}} \phi_{w^{( \pm)}}, \quad\left[V_{0}\right]_{i j} \equiv \partial_{v_{i}} \phi_{w^{(0)}}, \quad\left[V_{v}\right]_{i j} \equiv \partial_{v_{i}} \phi_{v_{j}} \text {. }}
\end{aligned}
$$

To proceed, we use the following relations that are valid when the selection rules are satisfied,

$$
\begin{aligned}
& U_{u}=V_{v}, \quad U_{v}=V_{u}, \quad W_{ \pm u}=W_{\mp v}, \quad U_{ \pm}=V_{\mp}, \quad U_{0}=V_{0}, \\
& W_{+ \pm}=W_{- \pm}, \quad W_{0 u}=W_{0 v}, \quad W_{0+}=W_{0-}, \quad W_{+0}=W_{-0} .
\end{aligned}
$$


Then we can rewrite the determinant by adding the rows and subtracting the columns.

$$
\begin{aligned}
\operatorname{det} G=\operatorname{det}\left(\begin{array}{ccccc}
U_{u} & U_{+} & U_{0} & U_{v} & U_{-} \\
W_{+u} & W_{++} & W_{+0} & W_{+v} & W_{+-} \\
W_{0 u} & W_{0+} & W_{00} & W_{0 v} & W_{0+} \\
U_{v} & U_{-} & U_{0} & U_{u} & U_{+} \\
W_{+v} & W_{+-} & W_{+0} & W_{+u} & W_{++}
\end{array}\right) \\
=\operatorname{det}\left(\begin{array}{ccccc}
U_{u}+U_{v} & U_{+}+U_{-} & 2 U_{0} & 0 & 0 \\
W_{+u}+W_{+v} & W_{++}+W_{+-} & W_{+0} & 0 & 0 \\
W_{0 u} & W_{0+} & W_{00} & 0 & 0 \\
U_{v} & U_{-} & U_{0} & U_{u}-U_{v} & U_{+}-U_{-} \\
W_{+v} & W_{+-} & W_{+0} & W_{+u}-W_{+v} & W_{++}-W_{+-}
\end{array}\right) .
\end{aligned}
$$

We thus obtain the factorization formula for the Gaudin determinant of the paritysymmetric states $\operatorname{det} G=\operatorname{det} G_{+} \operatorname{det} G_{-}$with

$$
\begin{aligned}
& \text { Odd } K_{\mathbf{w}}: \quad G_{+}=\left(\begin{array}{ccc}
U_{u}+U_{v} & U_{+}+U_{-} & 2 U_{0} \\
W_{+u}+W_{+v} & W_{++}+W_{+-} & 2 W_{+0} \\
W_{0 u} & W_{0+} & W_{00}
\end{array}\right), \\
& G_{-}=\left(\begin{array}{cc}
U_{u}-U_{v} & U_{+}-U_{-} \\
W_{+u}-W_{+v} & W_{++}-W_{+-}
\end{array}\right) .
\end{aligned}
$$

For the even $K_{\mathrm{w}}$ cases, we can repeat the derivation above simply by omitting the row and the colum involving $\mathbf{w}_{0}$. As a result we obtain the following factorization formula $\operatorname{det} G=\operatorname{det} G_{+} \operatorname{det} G_{-}$;

$$
\begin{aligned}
& \text { Even } K_{\mathbf{w}}: \quad G_{+}=\left(\begin{array}{cc}
U_{u}+U_{v} & U_{+}+U_{-} \\
W_{+u}+W_{+v} & W_{++}+W_{+-}
\end{array}\right), \\
& G_{-}=\left(\begin{array}{cc}
U_{u}-U_{v} & U_{+}-U_{-} \\
W_{+u}-W_{+v} & W_{++}-W_{+-}
\end{array}\right) .
\end{aligned}
$$

The same submatrices $G_{ \pm}$appear in the expression for the overlap (5.5).

To summarize, the norm of the parity-symmetric state is given by

$$
\langle\mathbf{u}, \mathbf{w},-\mathbf{u} \mid \mathbf{u}, \mathbf{w},-\mathbf{u}\rangle=\prod_{k=1}^{\left\lceil\frac{K_{\mathbf{w}}}{2}\right\rceil} \frac{\left(w_{k}-\frac{i}{2}\right)\left(w_{k}^{*}+\frac{i}{2}\right)}{\left(w_{k}+\frac{i}{2}\right)\left(w_{k}^{*}-\frac{i}{2}\right)} \prod_{j=1}^{K_{\mathbf{u}}}\left(u_{j}^{2}+\frac{1}{4}\right)^{2} \operatorname{det} G_{+} \operatorname{det} G_{-} .
$$

\subsection{Some details on the overlap}

We now explain the details of how we arrive at the formula (5.5). For this purpose, let us first present our conjecture for the overlap $\langle\mathcal{B} \mid \mathbf{u}, \mathbf{w},-\mathbf{u}\rangle$ itself:

$$
\langle\mathcal{B} \mid \mathbf{u}, \mathbf{w},-\mathbf{u}\rangle=2(-1)^{L} \prod_{j=1}^{K_{\mathbf{u}}}\left(u_{j}^{2}+\frac{1}{4}\right)\left(u_{j}+\frac{i}{2}\right) \prod_{k=1}^{\left\lceil\frac{K_{\mathbf{w}}}{2}\right\rceil} \frac{1}{w_{k}\left(w_{k}+\frac{i}{2}\right)} \operatorname{det} G_{+} .
$$


We stress that the state $|\mathbf{u}, \mathbf{w},-\mathbf{u}\rangle$ was constructed using the nested coordinate Bethe ansatz, with the order of the magnon rapidities given by (5.4).

Combining this with the result for the norm of the parity-symmetric state (5.18), we obtain

$$
\begin{aligned}
& \frac{\langle\mathcal{B} \mid \mathbf{u}, \mathbf{w},-\mathbf{u}\rangle}{\sqrt{\langle\mathbf{u}, \mathbf{w},-\mathbf{u} \mid \mathbf{u}, \mathbf{w},-\mathbf{u}\rangle}} \\
& \quad=2(-1)^{L} \prod_{j=1}^{K_{\mathbf{u}}}\left(u_{j}+\frac{i}{2}\right) \prod_{k=1}^{\left\lceil\frac{K_{\mathbf{w}}}{2}\right\rceil} \frac{1}{w_{k}\left(w_{k}+\frac{i}{2}\right)}\left[\prod_{k=1}^{\left\lceil\frac{K_{\mathbf{w}}}{2}\right\rceil} \frac{\left(w_{k}+\frac{i}{2}\right)\left(w_{k}^{*}-\frac{i}{2}\right)}{\left(w_{k}-\frac{i}{2}\right)\left(w_{k}^{*}+\frac{i}{2}\right)}\right]^{\frac{1}{4}} \sqrt{\frac{\operatorname{det} G_{+}}{\operatorname{det} G_{-}}} .
\end{aligned}
$$

To arrive at the formula (5.5), we then utilize the phase ambiguity discussed in section 2.2. For instance, multiplying the following phase ${ }^{15}$

$$
\begin{aligned}
e^{i \varphi} & \equiv(-1)^{L+1}\left(\prod_{k=1}^{\left\lceil\frac{K_{\mathbf{w}}}{2}\right\rceil} \frac{\left(w_{k}+\frac{i}{2}\right)\left(w_{k}^{*}+\frac{i}{2}\right)}{\left(w_{k}-\frac{i}{2}\right)\left(w_{k}^{*}-\frac{i}{2}\right)}\right)^{\frac{1}{4}} \prod_{j=1}^{K_{\mathbf{u}}}\left(\frac{u_{j}-\frac{i}{2}}{u_{j}^{*}+\frac{i}{2}}\right)^{\frac{1}{2}} \\
& =(-1)^{L+1}\left(\prod_{k=1}^{\left\lceil\frac{K_{\mathbf{w}}}{2}\right\rceil} \frac{\left(w_{k}+\frac{i}{2}\right)\left(w_{k}^{*}+\frac{i}{2}\right)}{\left(w_{k}-\frac{i}{2}\right)\left(w_{k}^{*}-\frac{i}{2}\right)}\right)^{\frac{1}{4}} \prod_{j=1}^{K_{\mathbf{u}}}\left(\frac{u_{j}-\frac{i}{2}}{u_{j}+\frac{i}{2}}\right)^{\frac{1}{2}},
\end{aligned}
$$

we get

$$
\frac{e^{i \varphi}\langle\mathcal{B} \mid \mathbf{u}, \mathbf{w},-\mathbf{u}\rangle}{\sqrt{\langle\mathbf{u}, \mathbf{w},-\mathbf{u} \mid \mathbf{u}, \mathbf{w},-\mathbf{u}\rangle}}=-2 \sqrt{\prod_{j=1}^{K_{\mathbf{u}}}\left(u_{j}^{2}+\frac{1}{4}\right) \prod_{k=1}^{\left\lceil\frac{K_{\mathbf{w}}}{2}\right\rceil} \frac{1}{w_{k}^{2}\left(w_{k}^{2}+\frac{1}{4}\right)} \frac{\operatorname{det} G_{+}}{\operatorname{det} G_{-}}}
$$

which coincides with (5.5).

\section{Conclusion}

In this paper, we studied the tree-level structure constants of a single-trace non-BPS operator and two sub-determinant operators (also known as giant gravitons) in ABJM theory in the planar limit. Much like in $\mathcal{N}=4 \mathrm{SYM}$, these structure constants can be computed by overlaps between a matrix product state and a Bethe eigenstate. To evaluate them explicitly, we developed the coordinate Bethe ansatz for the alternating SU(4) spin chain. As a result of the computation, we found that the overlap obeys a selection rule similar to the ones found in defect one-point functions in $\mathcal{N}=4 \mathrm{SYM}$. We also found a closed-form expression for the overlaps for the maximal giant gravitons.

This paper is the first installment of our studies of the structure constants of giant gravitons in ABJM theory. In the second paper [31], we will analyze these quantities at

\footnotetext{
${ }^{15}$ Here we used the fact that $\mathbf{u}$ as a set must be invariant under the complex conjugation (otherwise, the energy and the higher conserved charges generically will take complex values). Similar results for XXX and XXZ spin chains were proved in [106].
} 
strong coupling using a holographic description. The results of this paper and the next will be used in the third paper [32] to test and verify the nonperturbative approach based on the integrable bootstrap.

There are several future directions worth pursuing. First it would be desirable to prove the determinant formula for the overlap conjectured in this paper. One possible strategy is to map it to a partition function of a lattice model (a vertex model) as was done for $\mathcal{N}=4$ SYM [42]. Another possible strategy is to use the algebraic Bethe ansatz and Separation of Variables ${ }^{16}$ following the recent work [109]. Second the coordinate Bethe ansatz developed in this paper will be useful for computing other quantities in ABJM theory, most notably the three-point functions of single-trace operators ${ }^{17}$. Such computations will provide valuable data for developing the hexagon formalism for ABJM theory, which is yet to be established. Works in this direction are in progress. Third it is important to perform the computation at a loop level in order to see if the structure found in this paper persists, and to have more data to test the bootstrap approach discussed in the third paper. Finally the results in this paper suggest that non-maximal giant gravitons in ABJM theory might also correspond to integrable boundary states. It would be interesting to explore this further ${ }^{18}$, e.g. by analyzing the boundary condition of the string worldsheet at strong coupling [62, 63] or by computing the Hamiltonian of the open spin chain attached to the non-maximal giant gravitons (see [113-115] for the results on the maximal giant graviton). In addition, it would be desirable to derive a closed-form expression for the overlap for the non-maximal giant gravitons. Another interesting question is to generalize the analysis in this paper to the dual giant gravitons and see whether they lead to integrable boundary conditions.

\section{Acknowledgments}

We are very thankful to Yang Zhang for kindly providing us computation resource. We would also like to thank Junpeng Cao and De-Liang Zhong for very helpful discussions. The work of PY and JBW is supported in part by the National Natural Science Foundation of China, Grant No. 11975164, 11935009, 12047502, 11947301, and Natural Science Foundation of Tianjin under Grant No. 20JCYBJC00910. The work of SK was supported in part by DOE grant number DE-SC0009988.

\section{A Partially contracted giant graviton}

In this appendix, we study the structure constants of two giant graviton and one single-trace operator of length $2 L$ using the partially-contracted-giant-graviton (PCGG) approach [29] in order to cross-check the results in the main text. For simplicity, we only discuss the maximal giant gravitons but the analysis can be readiliy extended to non-maximal giant gravitons.

\footnotetext{
${ }^{16}$ The relation between overlaps of integrable boundary states and Separation of Variables was studied also in recent papers $[107,108]$ from different perspectives.

${ }^{17} \mathrm{See}[110]$ for results in the $\mathrm{SU}(2) \times \mathrm{SU}(2)$ sector.

${ }^{18}$ It would also be useful to revisit the analysis in [77] in order to understand the difference between $\mathcal{N}=4 \mathrm{SYM}$ and ABJM. This is important since, even for $\mathcal{N}=4 \mathrm{SYM}$, there are contradicting claims on the (non-)integrability of the non-maximal giant gravitons in the literature [60,61] (see also [111, 112]).
} 
Two-point function. The main focus of this appendix is the three-point function

$$
G_{2}=\left\langle\mathcal{D}_{1}\left(x_{1}\right) \mathcal{D}_{2}\left(x_{2}\right) \mathcal{O}\left(x_{3}\right)\right\rangle
$$

where

$$
\mathcal{D}_{i}\left(x_{i}\right)=\operatorname{det}\left(\left(n_{i} \cdot Y\right)\left(\bar{n}_{i} \cdot \bar{Y}\right)\right)=\operatorname{det}\left(n_{i} \cdot Y\right) \operatorname{det}\left(\bar{n}_{i} \cdot \bar{Y}\right) .
$$

However, it is useful to first analyze the two-point function

$$
\left\langle\mathcal{D}_{1}\left(x_{1}\right) \mathcal{D}_{2}\left(x_{2}\right)\right\rangle=\left\langle\operatorname{det}\left(n_{1} \cdot Y\right) \operatorname{det}\left(\bar{n}_{2} \cdot \bar{Y}\right)\right\rangle\left\langle\operatorname{det}\left(n_{2} \cdot Y\right) \operatorname{det}\left(\bar{n}_{1} \cdot \bar{Y}\right)\right\rangle .
$$

At the leading order, we can compute it by Wick contraction. The propagator is given by

$$
\left\langle\left(n_{1} \cdot Y(x)\right)_{a}^{\bar{b}}\left(\bar{n}_{2} \cdot \bar{Y}(y)\right)_{\bar{c}}{ }^{d}\right\rangle=\frac{1}{k} \frac{n_{1} \cdot \bar{n}_{2}}{|x-y|} \delta_{a}^{d} \delta_{\bar{c}}^{\bar{b}} .
$$

The determinant can be written as

$$
\operatorname{det} X=\frac{1}{N !} \epsilon_{a_{1} \cdots a_{N}} \epsilon^{b_{1} \cdots b_{N}} X_{b_{1}}^{a_{1}} \cdots X_{b_{N}}^{a_{N}} .
$$

Therefore, the two-point function of a single determinant is given by

$$
\begin{aligned}
\left\langle\operatorname{det}\left(n_{1} \cdot Y\right)\left(x_{1}\right) \operatorname{det}\left(\bar{n}_{2} \cdot \bar{Y}\right)\left(x_{2}\right)\right\rangle= & \frac{1}{k^{N}(N !)^{2}} \epsilon_{a_{1} \cdots a_{N}} \epsilon^{b_{1} \cdots b_{N}} \epsilon_{c_{1} \cdots c_{N}} \epsilon^{d_{1} \cdots d_{N}} \\
& \times \frac{\left(n_{1} \cdot \bar{n}_{2}\right)^{N}}{\left|x_{12}\right|^{N}} \delta_{a_{1}}^{d_{1}} \cdots \delta_{a_{N}}^{d_{N}} \delta_{b_{1}}^{c_{1}} \cdots \delta_{b_{N}}^{c_{N}} \cdot N ! \\
= & \frac{\left(n_{1} \cdot \bar{n}_{2}\right)^{N} N !}{k^{N}\left|x_{12}\right|^{N}}=\frac{d_{12}^{N}}{k^{N}} N !,
\end{aligned}
$$

where we have used $\epsilon_{a_{1} \cdots a_{N}} \epsilon^{a_{1} \cdots a_{N}}=N$ !. Then, we get the following result for the two-point function of giant gravitons

$$
\left\langle\mathcal{D}_{1}\left(x_{1}\right) \mathcal{D}_{2}\left(x_{2}\right)\right\rangle=\frac{1}{k^{2 N}}\left(d_{12} d_{21}\right)^{N}(N !)^{2} .
$$

Partially contracted giant graviton. To compute the three-point function involving a single-trace operator of length $2 L$ (A.1), we first perform the Wick contractions between the two giant gravitons partially so that it leaves precisely $2 L$ uncontracted scalar fields. We then perform the Wick contraction between these scalar fields with the single trace operator. We see that, in ABJM theory, the giant graviton factorizes into the product of two determinants (A.2). In the large $N$ limit, the partially contracted giant graviton at the leading order is given by the sum of the following two contributions:

- Contract completely $\operatorname{det}\left(n_{1} \cdot Y\right)$ and $\operatorname{det}\left(\bar{n}_{2} \cdot \bar{Y}\right)$ and take the leading term of the partially contracted $\operatorname{det}\left(n_{2} \cdot Y\right)$ and $\operatorname{det}\left(\bar{n}_{1} \cdot \bar{Y}\right)$.

- Contract completely $\operatorname{det}\left(n_{2} \cdot Y\right)$ and $\operatorname{det}\left(\bar{n}_{1} \cdot \bar{Y}\right)$ and take the leading term of the partially contracted $\operatorname{det}\left(n_{1} \cdot Y\right)$ and $\operatorname{det}\left(\bar{n}_{2} \cdot \bar{Y}\right)$. 
The leading term of the partially contracted determinant operators have been worked out in [29] in the study of giant gravitons in $\mathcal{N}=4 \mathrm{SYM}$, and we can simply recycle the result. For instance, the PCGG obtained from the partial contraction of $\operatorname{det}\left(n_{2} \cdot Y\right)$ and $\operatorname{det}\left(\bar{n}_{1} \cdot \bar{Y}\right)$ is a non-local single trace operator

$$
(N-L) ! \frac{\left(d_{21}\right)^{N-L}}{k^{N-L}} \frac{(-1)^{L+1}}{L} \operatorname{tr}\left[\left(\left(n_{2} \cdot Y\right)\left(x_{2}\right)\left(\bar{n}_{1} \cdot \bar{Y}\right)\left(x_{1}\right)\right)^{L}\right] .
$$

A similar expression can be derived for the partial contraction of $\operatorname{det}\left(n_{1} \cdot Y\right)$ and $\operatorname{det}\left(\bar{n}_{2} \cdot \bar{Y}\right)$. Combining the two contributions, we find that the full PCGG is given by

$$
\begin{gathered}
G_{L}\left(x_{1}, x_{2}\right)=N !(N-L) ! \frac{\left(d_{21} d_{12}\right)^{N}}{k^{2 N-L}} \frac{(-1)^{L+1}}{L}\left(\frac{\operatorname{tr}\left[\left(\left(n_{2} \cdot Y\right)\left(x_{2}\right)\left(\bar{n}_{1} \cdot \bar{Y}\right)\left(x_{1}\right)\right)^{L}\right]}{\left(d_{21}\right)^{L}}\right. \\
\left.+\frac{\operatorname{tr}\left[\left(\left(n_{1} \cdot Y\right)\left(x_{1}\right)\left(\bar{n}_{2} \cdot \bar{Y}\right)\left(x_{2}\right)\right)^{L}\right]}{\left(d_{12}\right)^{L}}\right) .
\end{gathered}
$$

In order to take the large $N$ limit, it is useful to divide it by the two-point function of giant gravitons (A.7). We then get

$$
\begin{aligned}
& \frac{G_{L}\left(x_{1}, x_{2}\right)}{\left\langle\mathcal{D}_{1}\left(x_{1}\right) \mathcal{D}_{2}\left(x_{2}\right)\right\rangle} \stackrel{N \rightarrow \infty}{=} \frac{(-1)^{L+1}}{L}\left(\frac{1}{\lambda}\right)^{L}\left(\frac{\operatorname{tr}\left[\left(\left(n_{2} \cdot Y\right)\left(x_{2}\right)\left(\bar{n}_{1} \cdot \bar{Y}\right)\left(x_{1}\right)\right)^{L}\right]}{\left(d_{21}\right)^{L}}\right. \\
& \left.+\frac{\operatorname{tr}\left[\left(\left(n_{1} \cdot Y\right)\left(x_{1}\right)\left(\bar{n}_{2} \cdot \bar{Y}\right)\left(x_{2}\right)\right)^{L}\right]}{\left(d_{12}\right)^{L}}\right) .
\end{aligned}
$$

Twisted-translated frame and MPS. To compare this with the results in the main text, we consider the twisted-translated kinematics and set $a_{1}=-a_{2}=1$ and $a_{3}=0$. Computing the Wick contractions between the PCGG (A.9) and the single-trace operator and dividing the result by the normalization $\mathcal{N}_{\mathcal{O}}$, we find that the structure constant is given by

$$
\mathfrak{D}_{N \mid \mathcal{O}}=-\frac{(-i)^{J}}{2^{\Delta-J}} \frac{\langle\mathcal{B} \mid \mathcal{O}\rangle}{\sqrt{L\langle\mathcal{O} \mid \mathcal{O}\rangle}}
$$

which precisely matches with the result in the main text (4.65).

\section{B Derivation of BPS three-point funcitons}

Here we explain how to derive the expression for the three-point function of two (non)maximal giant gravitons and a single-trace BPS operator given in (4.51). 
The starting point of the analysis is the generating function of structure constants (4.50). In order to extract the structure constant for a single-trace operator with a fixed charge, we perform the integration over $s$; namely

$$
\frac{\left\langle\mathcal{D}_{M} \mathcal{D}_{M} \mathcal{O}_{L}^{\circ}\right\rangle}{\left\langle\mathcal{D}_{M} \mathcal{D}_{M}\right\rangle}=-\oint_{|s|=\epsilon \ll 1} \frac{d s}{2 \pi i s^{1+L}} \oint_{|z|=1} \frac{d z}{2 \pi i z} \operatorname{tr}_{2}\left[\frac{1}{1-s \mathcal{T}_{\theta} \overline{\mathcal{T}}_{\theta}}\right]
$$

Here we replaced the integration variable $\theta$ with $z \equiv e^{i \theta}$. Note also that the integration of $s$ is performed in a region near the origin $|s| \ll 1$ since the generating function $1 /\left(1-s \overline{\mathcal{T}}_{\theta} \overline{\mathcal{T}}_{\theta}\right)$ is expected to have a finite radius of convergence when expanded in $s$ and therefore one has to take $|s|$ to be sufficiently small in order to use the formula.

As the next step, we diagonalize the matrix $1 /\left(1-s \mathcal{T}_{\theta} \overline{\mathcal{T}}_{\theta}\right)$. We also change the integration variables as

$$
\tilde{s}=\lambda \frac{d_{13} d_{32}}{d_{12}} s, \quad \tilde{z}=\left(\frac{d_{13} d_{31}}{d_{23} d_{32}}\right)^{\frac{1}{4}} z
$$

in order to simplify the expression. As a result we get

$$
\begin{aligned}
\frac{\left\langle\mathcal{D}_{M} \mathcal{D}_{M} \mathcal{O}_{L}^{\circ}\right\rangle}{\left\langle\mathcal{D}_{M} \mathcal{D}_{M}\right\rangle}= & -\left(\lambda \frac{d_{13} d_{32}}{d_{12}}\right)^{L} \oint_{|\tilde{s}|=\epsilon \ll 1} \frac{d \tilde{s}}{2 \pi i \tilde{s}^{1+L}} \oint_{|z|=1} \frac{d \tilde{z}}{2 \pi i \tilde{z}} \\
& \times\left[1+\frac{\tilde{z}^{2}\left(1-\xi \tilde{s}^{2}\right)}{\sqrt{\xi} \tilde{s}(\omega-1)\left(1+\tilde{z}^{4}\right)+\tilde{z}^{2}((\xi+1) \tilde{s} \omega-2)}\right]^{-1} .
\end{aligned}
$$

We then perform the integration of $\tilde{z}$ by closing the contour and picking up contributions from poles inside the contour. When $|\tilde{s}| \ll 1$, we find that the integrand has three poles inside the contour $|z|=1$ :

$$
\tilde{z}=0, \quad \pm \frac{1}{\xi^{1 / 4}} \sqrt{\frac{1+\tilde{s}^{2} \xi-(1+\xi) \tilde{s} \omega-\sqrt{\left(1+\tilde{s}^{2} \xi-\tilde{s}(1+\xi) \omega\right)^{2}-4 \tilde{s}^{2} \xi(\omega-1)^{2}}}{2 \tilde{s}(\omega-1)}} .
$$

Computing the residues from these poles (and redefining $\tilde{s}$ as $s$ ), we obtain (4.51).

\section{Data and numerics}

In this appendix, we explain our method to numerically solve the Bethe equation and provide some data for the overlap with the non-maximal giant graviton.

\section{C.1 Numerical solutions to the Bethe equations}

Solving the Bethe equation. To solve the Bethe equations (3.7) numerically, we follow the following strategy: first we divide the auxiliary roots $\mathbf{w}$ into two subsets $\mathbf{w}=\mathbf{w}^{(1)} \cup \mathbf{w}^{(2)}$, 
introduce a parameter $\epsilon$ to modify the Bethe equations into the following:

$$
\begin{aligned}
& \left(\frac{u_{j}+\frac{i}{2}}{u_{j}-\frac{i}{2}}\right)^{L}=\prod_{\substack{k=1 \\
k \neq j}}^{K_{\mathbf{u}}} \frac{u_{j}-u_{k}+i}{u_{j}-u_{k}-i} \prod_{k=1}^{K_{\mathrm{w}}^{(1)}} \frac{u_{j}-w_{k}^{(1)}-\frac{i}{2}}{u_{j}-w_{k}^{(1)}+\frac{i}{2}} \prod_{k=1}^{K_{\mathrm{w}}^{(2)}} \frac{u_{j}-w_{k}^{(2)}-\frac{i}{2} \epsilon}{u_{j}-w_{k}^{(2)}+\frac{i}{2} \epsilon}, \\
& \left(\frac{v_{j}+\frac{i}{2}}{v_{j}-\frac{i}{2}}\right)^{L}=\prod_{\substack{k=1 \\
k \neq j}}^{K_{\mathbf{v}}} \frac{v_{j}-v_{k}+i}{v_{j}-v_{k}-i} \prod_{k=1}^{K_{\mathrm{w}}^{(1)}} \frac{v_{j}-w_{k}^{(1)}-\frac{i}{2}}{v_{j}-w_{k}^{(1)}+\frac{i}{2}} \prod_{k=1}^{K_{\mathrm{w}}^{(2)}} \frac{v_{j}-w_{k}^{(2)}-\frac{i}{2} \epsilon}{v_{j}-w_{k}^{(2)}+\frac{i}{2} \epsilon}, \\
& 1=\prod_{k=1}^{K_{\mathbf{u}}} \frac{w_{j}^{(1)}-u_{k}-\frac{i}{2}}{w_{j}^{(1)}-u_{k}+\frac{i}{2}} \prod_{k=1}^{K_{\mathbf{v}}} \frac{w_{j}^{(1)}-v_{k}-\frac{i}{2} \epsilon}{w_{j}^{(1)}-v_{k}+\frac{i}{2} \epsilon} \\
& \times \prod_{\substack{k=1 \\
k \neq j}}^{K_{\mathrm{w}}^{(1)}} \frac{w_{j}^{(1)}-w_{k}^{(1)}+i}{w_{j}^{(1)}-w_{k}^{(1)}-i} \prod_{k=1}^{K_{\mathrm{w}}^{(2)}} \frac{w_{j}^{(1)}-w_{k}^{(2)}+i \epsilon}{w_{j}^{(1)}-w_{k}^{(2)}-i \epsilon} \\
& 1=\prod_{k=1}^{M} \frac{w_{j}^{(2)}-u_{k}-\frac{i}{2} \epsilon}{w_{j}^{(2)}-u_{k}+\frac{i}{2} \epsilon} \prod_{k=1}^{N} \frac{w_{j}^{(2)}-v_{k}-\frac{i}{2}}{w_{j}^{(2)}-v_{k}+\frac{i}{2}} \\
& \times \prod_{\substack{k=1 \\
k \neq j}}^{K_{\mathrm{w}}^{(2)}} \frac{w_{j}^{(2)}-w_{k}^{(2)}+i}{w_{j}^{(2)}-w_{k}^{(2)}-i} \prod_{k=1}^{K_{\mathrm{w}}^{(1)}} \frac{w_{j}^{(2)}-w_{k}^{(1)}+i \epsilon}{w_{j}^{(2)}-w_{k}^{(1)}-i \epsilon} .
\end{aligned}
$$

The modified Bethe equations (C.1) go back to the original equations when $\epsilon=1$ while they become two decoupled $\mathrm{SU}(3)$ Bethe equations when $\epsilon=0$.

Second we solve the SU(3) Bethe equations using the rational Q-system ${ }^{19}$ by Marboe and Volin [117]. We then use these solutions as "seed" solutions for $\epsilon=0$ and use FindRoots in Mathematica to generate solutions for $\epsilon=1$.

As a result, we obtained the solutions to the original Bethe equations (3.7). In what follows we only consider the solutions with $K_{\mathbf{u}}=K_{\mathbf{v}}=K_{\mathbf{w}}$ that are relevant for the computation of the overlap. Note also that the solutions listed below are by no means exhaustive. It would be desirable to improve the algorithm for solving the Bethe equations and perform more systematic checks. In particular, the generalization of Marboe-Volin algorithm for the alternating spin chain is an important open problem.

Solutions to the Bethe equations. Let us now list the solutions we obtained. Here the Bethe roots denoted in red satisfy both the selection rules and the zero-momentum condition while the Bethe roots denoted in blue only satisfy the zero-momentum condition. All the other Bethe roots do not satisfy the zero-momentum condition and therefore do not correspond to single-trace operators in ABJM theory. For later convenience, we also numbered each solution in red, as $[\mathrm{n}]$.

\footnotetext{
${ }^{19}$ However, the $\mathrm{Q}$ system there only works for the case when $L-M \geq M-N \geq N$, where $L$ is the length of the $\mathrm{SU}(3)$ spin chain and $M, N$ are number of two types of magnons. Sometimes this constraint is too strong to obtain the $\mathrm{SU}(4)$ solutions. In some cases, we can fix this problem using the bosonic duality [116].
} 


\begin{tabular}{|c|c|c|}
\hline$L$ & $K_{\mathbf{w}}$ & {$[\mathbf{u}, \mathbf{v}, \mathbf{w}]$} \\
\hline 1 & 1 & {$[\{0\},\{0\},\{0\}][1]$} \\
\hline \multirow[t]{3}{*}{2} & \multirow[t]{3}{*}{1} & {$\left[\left\{\frac{1}{2 \sqrt{3}}\right\},\left\{-\frac{1}{2 \sqrt{3}}\right\},\{0\}\right]$} \\
\hline & & {$\left[\left\{\frac{1}{2}\right\},\left\{\frac{1}{2}\right\},\left\{\frac{1}{2}\right\}\right]$} \\
\hline & & {$\left[\left\{-\frac{1}{2}\right\},\left\{-\frac{1}{2}\right\},\left\{-\frac{1}{2}\right\}\right]$} \\
\hline \multirow[t]{3}{*}{3} & \multirow[t]{3}{*}{1} & {$[\{0\},\{0\},\{0\}][2]$} \\
\hline & & {$\left[\left\{\frac{1}{2}\right\},\left\{-\frac{1}{2}\right\},\{0\}\right][3]$} \\
\hline & & {$\left[\left\{-\frac{\sqrt{3}}{2}\right\},\left\{-\frac{\sqrt{3}}{2}\right\},\left\{-\frac{\sqrt{3}}{2}\right\}\right.$} \\
\hline 4 & 1 & {$\left[\left\{\frac{\sqrt{7}-2}{6}\right\},\left\{-\frac{\sqrt{7}+2}{6}\right\},\left\{-\frac{1}{3}\right\}\right.$} \\
\hline \multirow[t]{2}{*}{5} & \multirow[t]{2}{*}{1} & {$[\{0\},\{0\},\{0\}][4]$} \\
\hline & & {$\left[\left\{-\frac{1}{\sqrt{12}}\right\},\left\{\frac{1}{\sqrt{12}}\right\},\{0\}\right][5]$} \\
\hline \multirow[t]{4}{*}{6} & \multirow[t]{4}{*}{1} & {$[\{-0.3987366944412021\},\{0.3987366944412021\},\{0\}]$} \\
\hline & & {$[\{0.13397459621556135\},\{0.13397459621556138\},\{0.13397459621556135\}]$} \\
\hline & & {$[\{0.45440762733328904\},\{0.15990657179334503\},\{0.307157099563317\}]$} \\
\hline & & {$\left[\left\{\frac{1}{2}\right\},\left\{\frac{1}{2}\right\},\left\{\frac{1}{2}\right\}\right]$} \\
\hline 2 & 2 & {$\left[\left\{\sqrt{\frac{3}{20}},-\sqrt{\frac{3}{20}}\right\},\left\{-\sqrt{\frac{3}{20}}, \sqrt{\frac{3}{20}}\right\},\left\{\frac{1}{\sqrt{5}},-\frac{1}{\sqrt{5}}\right\}\right][6]$} \\
\hline \multirow[t]{3}{*}{3} & \multirow[t]{3}{*}{2} & $\begin{array}{l}{[\{-0.34554024732023516,0.34554024732023516\},} \\
\{0.34554024732023516,-0.34554024732023516\}, \\
\{0.51108108452939387 i,-0.51108108452939387 i\}]\end{array}$ \\
\hline & & $\begin{array}{l}{[\{-0.51320279936202029,0.062678427970585504\},} \\
\{0.51320279936202029,-0.062678427970585504\}, \\
\{-0.41623324836102756,0.41623324836102756\}]\end{array}$ \\
\hline & & $\begin{array}{l}{[\{-0.67910322332093357,0.025611245506665032\},} \\
\{0.53448799950517913,-0.74702142547534923\}, \\
\{-0.76136871888869386,0.32835601699647453\}]\end{array}$ \\
\hline \multirow[t]{3}{*}{4} & \multirow[t]{3}{*}{2} & $\begin{array}{l}{[\{-0.56944513222254722,0.56944513222254722\},} \\
\{0.56944513222254722,-0.56944513222254722\} \\
\{0.55472575089337952 i,-0.55472575089337952 i\}][7]\end{array}$ \\
\hline & & $\begin{array}{l}{[\{-0.66931302374657110,-0.10647062449199778\},} \\
\{0.66931302374657110,0.10647062449199778\}, \\
\{-0.48978976193047651,0.48978976193047651\}][8]\end{array}$ \\
\hline & & $\begin{array}{l}{[\{-0.028299146374196785,0.55999384176787242\},} \\
\{-0.028299146374196785,0.55999384176787242\}, \\
\{0.26584734769683781-0.50624190637875312 i, \\
0.26584734769683781+0.50624190637875312 i\}]\end{array}$ \\
\hline
\end{tabular}




\begin{tabular}{|c|c|c|}
\hline \multirow[t]{2}{*}{6} & \multirow[t]{2}{*}{2} & $\begin{array}{l}{[\{0.34675919853122809,0.99366751548216189\},} \\
\{-0.34675919853122809,-0.99366751548216189\} \\
\{0.71060441235307684,-0.71060441235307684\}][9]\end{array}$ \\
\hline & & $\begin{array}{l}{[\{-1.0773108398074073,0.49798319908651183\}} \\
\{0.089846608200097142,0.42002515253515016\} \\
\{-0.59531374844468910,0.56058580845186502\}]\end{array}$ \\
\hline \multirow[t]{5}{*}{6} & \multirow[t]{5}{*}{2} & $\begin{array}{l}{[\{0.40946507692652007,0.10041044458589016\}} \\
\{-0.39457524195942078,0.15869120668880070\} \\
\{0.44210986670775702,-0.30511412358686194\}]\end{array}$ \\
\hline & & $\begin{array}{l}{[\{0.11567004621958770,0.44116926182762157\}} \\
\{-0.40026535781351243,0.48672949230748789\} \\
\{0.57166303815947364,-0.25001131688888128\}]\end{array}$ \\
\hline & & $\begin{array}{l}{[\{0.37830228050349018,1.0458410010838975\}} \\
\{-0.055390813251937810,-0.35320869561294462\} \\
\{0.78252761450158240,-0.27475572814032976\}]\end{array}$ \\
\hline & & $\begin{array}{l}{[\{0.36681212663617484,1.0519697940662345\}} \\
\{0.20899528319467480,-1.0040495234017774\} \\
\{0.82203932258846606,-0.51017548234081269\}]\end{array}$ \\
\hline & & $\begin{array}{l}{[\{0.13241485029334295,0.45759251757083131\}} \\
\{-0.098732170839668265,0.48070339312713823\} \\
\{0.59462910995790549,-0.10863981488208338\}]\end{array}$ \\
\hline 3 & 3 & $\begin{array}{l}{[\{-0.61842989257770833,0,0.61842989257770833\}} \\
\{0.61842989257770833,0,-0.61842989257770833\} \\
\{0.71410132990930250,-0.71410132990930250,0\}][10]\end{array}$ \\
\hline 5 & 3 & $\begin{array}{l}{[\{-0.90018200552947429,0.031648482693564728,0.37680381182936445\}} \\
\{0.90018200552947429,-0.031648482693564728,-0.37680381182936445\}, \\
\{-0.75676650863660755,0.75676650863660755,0\}][11]\end{array}$ \\
\hline \multirow[t]{2}{*}{6} & \multirow[t]{2}{*}{4} & $\begin{array}{l}{[\{ \pm 0.53714639382219377 \pm 0.51401134667644172 i\}} \\
\{\mp 0.53714639382219377 \mp 0.51401134667644172 i\} \\
\{ \pm 1.6004024229193260 i, \pm 0.50622703524559230 i\}]^{20} \quad[12]\end{array}$ \\
\hline & & $\begin{array}{l}{[\{-0.098635382965062615,0.14753207194168752,} \\
-1.0179905746843994,0.47877616601418219\}, \\
\{0.098635382965062615,-0.14753207194168752, \\
1.0179905746843994,-0.47877616601418219\}, \\
\{-0.89439365253962075,0.89439365253962075, \\
0.22001470275637868,-0.22001470275637868\}] \quad[13]\end{array}$ \\
\hline
\end{tabular}

\footnotetext{
${ }^{20}$ Here the precise meaning of the expression like " $\{ \pm \operatorname{Re}(u) \pm i \operatorname{Im}(u)\},\{\mp \operatorname{Re}(u) \mp i \operatorname{Im}(u)\},\left\{ \pm w_{1}, \pm w_{2}\right\}$ " is " $\{\operatorname{Re}(u)+i \operatorname{Im}(u),-\operatorname{Re}(u)+i \operatorname{Im}(u), \operatorname{Re}(u)-i \operatorname{Im}(u),-\operatorname{Re}(u)-i \operatorname{Im}(u)\},\{-\operatorname{Re}(u)-i \operatorname{Im}(u), \operatorname{Re}(u)-$ $i \operatorname{Im}(u),-\operatorname{Re}(u)+i \operatorname{Im}(u), \operatorname{Re}(u)+i \operatorname{Im}(u)\},\left\{w_{1},-w_{1}, w_{2},-w_{2}\right\} "$.
} 


\section{C.2 Overlaps for non-maximal giant gravitons}

Here we summarize the results for overlaps for non-maximal giant gravitons $\omega \neq 1$. Below we list the results for the ratio between the structure constant of the maximal giant graviton $\mathfrak{D}_{N \mid \mathcal{O}}$ and the structure constant of the non-maximal giant graviton $\mathfrak{D}_{M \mid \mathcal{O}}$; $r \equiv \mathfrak{D}_{M \mid \mathcal{O}} / \mathfrak{D}_{N \mid \mathcal{O}}$

$$
\begin{aligned}
{[1] \quad r=} & \omega \\
{[2] \quad r=} & -2 \omega^{3}+4 \omega^{2}-\omega, \\
{[3] \quad r=} & -2 \omega^{3}+4 \omega^{2}-\omega, \\
{[4] \quad r=} & 6 \omega^{5}-24 \omega^{4}+30 \omega^{3}-12 \omega^{2}+\omega \\
{[5] \quad r=} & 6 \omega^{5}-24 \omega^{4}+30 \omega^{3}-12 \omega^{2}+\omega, \\
{[6] \quad r=} & 2 \omega^{2}-\omega \\
{[7] \quad r=} & -4 \omega^{4}+8.80557589 \omega^{3}-3.77669700 \omega^{2}-0.028878886 \omega, \\
{[8] \quad r=} & -4 \omega^{4}+8.21090774 \omega^{3}-3.39983829 \omega^{2}+0.188930549 \omega, \\
{[9] \quad r=} & 12 \omega^{6}-47.8851733 \omega^{5}+62.8683251 \omega^{4}-30.0180998 \omega^{3} \\
& +3.96919074 \omega^{2}+0.065757247 \omega, \\
{[10] \quad r=} & -2 \omega^{3}+4 \omega^{2}-\omega, \\
{[11] \quad r=} & -8 \omega^{5}+20.17658812 \omega^{4}-12.92497528 \omega^{3} \\
& +1.74975464 \omega^{2}-0.001367480 \omega, \\
{[12] \quad r=} & -16 \omega^{6}+42.978665 \omega^{5}-33.996976 \omega^{4}+8.5586366 \omega^{3} \\
& -0.54003958 \omega^{2}-0.00028639193 \omega, \\
{[13] \quad r=} & -16 \omega^{6}+49.281471 \omega^{5}-51.190705 \omega^{4}+23.285677 \omega^{3} \\
& -5.0615656 \omega^{2}+0.68512348 \omega .
\end{aligned}
$$

Here $[\mathrm{n}]$ 's refer to the numbers in the table above.

\section{Nested algebraic Bethe ansatz}

In this appendix, we review the nested algebraic Bethe ansatz approach for the SU(4) alternating spin chain $[34,37]$. We begin with the following set of four $R$-matrices,

$$
\begin{aligned}
& R_{a b}=u+\mathrm{P}_{a b}, \\
& R_{\bar{a} \bar{b}}=u+\mathrm{P}_{\bar{a} \bar{b}}, \\
& R_{a \bar{b}}=-(u+2)+\mathrm{K}_{a \bar{b}}, \\
& R_{\bar{a} b}=-(u+2)+\mathrm{K}_{\bar{a} b},
\end{aligned}
$$

where $a(\bar{a})$ denotes the spin in the fundamental (anti-fundamental) representation of $\mathrm{SU}(4)$. These $R$-matrices satisfy the following eight Yang-Baxter equations,

$$
R_{\mathrm{ab}}(u-v) R_{\mathbf{a c}}(u) R_{\mathbf{b c}}(v)=R_{\mathbf{b c}}(v) R_{\mathbf{a c}}(u) R_{\mathbf{a b}}(u-v),
$$

where a can take value of $a$ or $\bar{a}$ independently. 
We now define two monodromy matrices using two different auxiliary spaces in $\mathbf{4}$ and $\overline{4}$ representations,

$$
\begin{aligned}
& T_{a}(u)=R_{a 1}(u) R_{a \overline{1}}(u) \cdots R_{a L}(u) R_{a \bar{L}}(u), \\
& T_{\bar{a}}(u)=R_{\bar{a} 1}(u) R_{\bar{a} \overline{1}}(u) \cdots R_{\bar{a} L}(u) R_{\bar{a} \bar{L}}(u) .
\end{aligned}
$$

The corresponding transfer matrices

$$
\tau(u)=\operatorname{Tr}_{a} T_{a}(u), \bar{\tau}(u)=\operatorname{Tr}_{\bar{a}} T_{\bar{a}}(u),
$$

commute with each other

$$
[\tau(u), \tau(v)]=0,[\bar{\tau}(u), \bar{\tau}(v)]=0,[\tau(u), \bar{\tau}(v)]=0,
$$

due to the above Yang-Baxter equations. We can generate two Hamiltonians from the transfer matrices,

$$
H_{\text {odd }}=\left.(\tau(0))^{-1} \frac{d}{d u} \tau(u)\right|_{u=0}, H_{\text {even }}=\left.(\bar{\tau}(0))^{-1} \frac{d}{d u} \bar{\tau}(u)\right|_{u=0} .
$$

The true Hamiltonian is given by $H=H_{\text {odd }}+H_{\text {even }}$ which is the same as planar two-loop anomalous dimension matrix in the scalar sector of ABJM theory, up to rescaling and shifting by a constant.

Due to (D.9), there exist $u$-indepedent common eigenstates of $\tau(u)$ and $\bar{\tau}(u)$. We now construct eigenstates of $\tau(u)$ using nested algebraic Bethe ansatz [118]. For a nice review, we refer to [119]. We first write the monodromy matrix $T_{a}(u)$ as $4 \times 4$ matrix whose elements are operators acting on the Hilbert space of the spin chain $\mathcal{H} \cong\left(\mathbf{C}^{4}\right)^{\otimes 2 L}$,

$$
T_{a}(u)=\left(\begin{array}{cccc}
A(u) & B_{1}(u) & B_{2}(u) & B_{3}(u) \\
C_{1}(u) & D_{22}(u) & D_{23}(u) & D_{24}(u) \\
C_{2}(u) & D_{32}(u) & D_{33}(u) & D_{34}(u) \\
C_{3}(u) & D_{42}(u) & D_{43}(u) & D_{44}(u)
\end{array}\right) .
$$

The RTT relation

$$
R_{a b}(u-v) T_{a}(u) T_{b}(v)=T_{b}(v) T_{a}(u) R_{a b}(u-v)
$$

from Yang-Bexter equations, leads to the following commutation relations,

$$
\begin{aligned}
A(u) B_{i}(v) & =\frac{u-v-1}{u-v} B_{i}(v) A(u)+\frac{1}{u-v} B_{i}(u) A(v), \\
D_{i j}(u) B_{k}(v) & =\frac{1}{u-v} B_{k^{\prime}}(v) D_{i j^{\prime}}(u)\left(R^{\mathrm{SU}(3)}(u-v)\right)_{k j}^{k^{\prime} j^{\prime}}-\frac{1}{u-v} B_{j}(u) D_{i k}(v),
\end{aligned}
$$

where $i, j, k, \cdots$ take values in $2,3,4$ and

$$
\left(R^{\mathrm{SU}(3)}(u-v)\right)_{k j}^{k^{\prime} j^{\prime}}=(u-v) \delta_{k}^{k^{\prime}} \delta_{j}^{j^{\prime}}+\delta_{j}^{k^{\prime}} \delta_{k}^{j^{\prime}}
$$


is an $\mathrm{SU}(3) \mathrm{R}$-matrix. And the red part in the above formulas is the origin of the unwanted terms in the following.

Now we consider the subspace $\mathcal{H}_{1}$ of $\mathcal{H}$ spanned by the state

$$
\left|1, \bar{i}_{1}, 1, \bar{i}_{2}, \cdots, 1, \bar{i}_{L}\right\rangle
$$

with $\bar{i}_{k}=2,3,4$. Any state $|1\rangle$ in this subspace satisfy the following important property,

$$
\begin{aligned}
A(u)|1\rangle & =(-u-2)^{L}(u+1)^{L}|1\rangle, \\
C_{i}(u)|1\rangle & =0, \\
D_{i j}(u)|1\rangle & \in \mathcal{H}_{1} .
\end{aligned}
$$

Consider the following state in $\mathcal{H}$,

$$
|\psi\rangle=B_{i_{1}}\left(\mu_{1}\right) \cdots B_{i_{M}}\left(\mu_{M}\right) X^{i_{1} \cdots i_{M}}|1\rangle,
$$

with $|1\rangle \in \mathcal{H}_{1}$ and $X^{i_{1} \cdots i_{M}}$ to-be-determined coefficients. The acts of $\tau(u)=A(u)+D_{j j}(u)$ on $|\psi\rangle$ is given by

$$
\begin{aligned}
A(u)|\psi\rangle= & (-u-2)^{L}(u+1)^{L} \prod_{i=1}^{M} \frac{u-\mu_{i}-1}{u-\mu_{i}}|\psi\rangle+\text { unwanted terms } \\
D_{j j}(u)|\psi\rangle= & \left(\prod_{i=1}^{M} \frac{1}{u-\mu_{i}}\right) B_{k_{1}}\left(\mu_{1}\right) \cdots B_{k_{M}}\left(\mu_{M}\right) D_{j l_{M}}(u) R^{\mathrm{SU}(3)}\left(u-\mu_{M}\right)_{i_{M} l_{M-1}}^{k_{M} l_{M}} \\
& \times R^{\mathrm{SU}(3)}\left(u-\mu_{M-1}\right)_{i_{M-1} l_{M-2}}^{k_{M-1} l_{M-1}} \cdots R^{\mathrm{SU}(3)}\left(u-\mu_{2}\right)_{i_{2} l_{1}}^{k_{2} l_{2}} R^{\mathrm{SU}(3)}\left(u-\mu_{1}\right)_{i_{1} j}^{k_{1} l_{1}} \\
& \times X^{i_{1} \cdots i_{M}}|1\rangle+\text { unwanted terms . }
\end{aligned}
$$

Now we need to compute the eigenstate of

$$
\begin{array}{r}
D_{j l_{M}}(u) R^{\mathrm{SU}(3)}\left(u-\mu_{M}\right)_{i_{M} l_{M-1}}^{k_{M} l_{M}} R^{\mathrm{SU}(3)}\left(u-\mu_{M-1}\right)_{i_{M-1} l_{M-2}}^{k_{M-1} l_{M-1}} \cdots R^{\mathrm{SU}(3)}\left(u-\mu_{2}\right)_{i_{2} l_{1}}^{k_{2} l_{2}} \\
\times R^{\mathrm{SU}(3)}\left(u-\mu_{1}\right)_{i_{1} j}^{k_{1} l_{1}}
\end{array}
$$

acting on $\left(\mathbf{C}^{3}\right)^{\otimes M} \otimes \mathcal{H}_{L}$. It is not hard to see that the problem is reduced to finding eigenvalue of transfer matrix on an integrable $\mathrm{SU}(3)$ spin chain with length $M+2 L$. On this chain, $\mu_{1}, \cdots, \mu_{M}$ play the roles of inhomogeneity parameters. One can reduce further to an $\mathrm{SU}(2)$ spin chain and finally diagonalize the transfer matrix. Collecting all of the wanted terms, we get

$$
\begin{aligned}
\Lambda(u)= & (-u-2)^{L}(u+1)^{L} \prod_{i=1}^{K_{\mathbf{u}}} \frac{u-\mu_{i}-1}{u-\mu_{i}} \\
& +(-u)^{L}(u+1)^{L} \prod_{j=1}^{K_{\mathbf{v}}} \frac{u-\nu_{j}+1}{u-\nu_{j}} \\
& +(-u)^{L}(u+2)^{L} \prod_{i=1}^{K_{\mathbf{u}}} \frac{u-\mu_{i}+1}{u-\mu_{i}} \prod_{k=1}^{K_{\mathbf{w}}} \frac{u-\lambda_{k}-1}{u-\lambda_{k}} \\
& +(-u)^{L}(u+2)^{L} \prod_{j=1}^{K_{\mathbf{v}}} \frac{u-\nu_{j}-1}{u-\nu_{j}} \prod_{k=1}^{K_{\mathbf{w}}} \frac{u-\lambda_{k}+1}{u-\lambda_{k}} .
\end{aligned}
$$


Demanding the vanishing of the residue of the spurious pole at $u=\mu_{i}, \nu_{j}, \lambda_{k}$ leads to Bethe equations. After performing the substitution $\mu_{i}=\mathrm{i} u_{i}-1 / 2, \nu_{j}=\mathrm{i} v_{j}-3 / 2, \lambda_{k}=\mathrm{i} w_{k}-1$, these Bethe equations becomes exact the Bethe ansatz equations (3.7) in subsection 3. When these Bethe equations are satisfied, the above $\Lambda(u)$ is the eigenvalue of $\tau(u)$, and the corresponding eigenstate can be constructed using the above procedure. We have checked, for small $L$, that such states are also eigenstates of $\bar{\tau}(u)$. It should be very valuable to prove this for the general case.

The Bethe states from algebraic Bethe ansatz and the ones from coordinate Bethe ansatz are related by

$$
|\mathbf{u}, \mathbf{v}, \mathbf{w}\rangle^{a l} \propto \prod_{i<j} \frac{u_{i}-u_{j}+\mathrm{i}}{u_{i}-u_{j}} \prod_{i<j} \frac{v_{i}-v_{j}+\mathrm{i}}{v_{i}-v_{j}} \prod_{i<j} \frac{w_{i}-w_{j}+\mathrm{i}}{w_{i}-w_{j}}|\mathbf{u}, \mathbf{v}, \mathbf{w}\rangle^{c o},
$$

with a proportional factor which is invariant under the permutations of rapidities of the same type.

Open Access. This article is distributed under the terms of the Creative Commons Attribution License (CC-BY 4.0), which permits any use, distribution and reproduction in any medium, provided the original author(s) and source are credited.

\section{References}

[1] J.A. Minahan and K. Zarembo, The Bethe ansatz for $N=4$ superYang-Mills, JHEP 03 (2003) 013 [hep-th/0212208] [INSPIRE].

[2] N. Gromov, V. Kazakov and P. Vieira, Exact spectrum of anomalous dimensions of planar $N=4$ supersymmetric Yang-Mills theory, Phys. Rev. Lett. 103 (2009) 131601 [arXiv:0901.3753] [INSPIRE].

[3] D. Bombardelli, D. Fioravanti and R. Tateo, Thermodynamic Bethe Ansatz for planar AdS/CFT: a proposal, J. Phys. A 42 (2009) 375401 [arXiv:0902.3930] [INSPIRE].

[4] G. Arutyunov and S. Frolov, Thermodynamic Bethe Ansatz for the $A d S_{5} \times S^{5}$ mirror model, JHEP 05 (2009) 068 [arXiv:0903.0141] [INSPIRE].

[5] N. Gromov, V. Kazakov, S. Leurent and D. Volin, Quantum spectral curve for planar $\mathcal{N}=4$ super-Yang-Mills theory, Phys. Rev. Lett. 112 (2014) 011602 [arXiv:1305.1939] [INSPIRE].

[6] N. Gromov, V. Kazakov, S. Leurent and D. Volin, Quantum spectral curve for arbitrary state/operator in $A d S_{5} / C F T_{4}, J H E P 09$ (2015) 187 [arXiv:1405.4857] [INSPIRE].

[7] B. Basso, S. Komatsu and P. Vieira, Structure constants and integrable bootstrap in planar $N=4 S Y M$ theory, arXiv: 1505.06745 [INSPIRE].

[8] T. Fleury and S. Komatsu, Hexagonalization of correlation functions, JHEP 01 (2017) 130 [arXiv: 1611.05577] [INSPIRE].

[9] B. Basso, V. Goncalves, S. Komatsu and P. Vieira, Gluing hexagons at three loops, Nucl. Phys. B 907 (2016) 695 [arXiv:1510.01683] [InSPIRE].

[10] B. Basso, F. Coronado, S. Komatsu, H.T. Lam, P. Vieira and D.-l. Zhong, Asymptotic four point functions, JHEP 07 (2019) 082 [arXiv: 1701.04462] [INSPIRE]. 
[11] B. Basso, V. Goncalves and S. Komatsu, Structure constants at wrapping order, JHEP 05 (2017) 124 [arXiv: 1702.02154] [INSPIRE].

[12] B. Eden and A. Sfondrini, Three-point functions in $\mathcal{N}=4 S Y M$ : the hexagon proposal at three loops, JHEP 02 (2016) 165 [arXiv:1510.01242] [INSPIRE].

[13] T. Fleury and S. Komatsu, Hexagonalization of correlation functions. Part II. Two-particle contributions, JHEP 02 (2018) 177 [arXiv: 1711.05327] [InSPIRE].

[14] B. Eden and A. Sfondrini, Tessellating cushions: four-point functions in $\mathcal{N}=4 S Y M$, JHEP 10 (2017) 098 [arXiv: 1611. 05436] [INSPIRE].

[15] F. Coronado, Perturbative four-point functions in planar $\mathcal{N}=4 S Y M$ from hexagonalization, JHEP 01 (2019) 056 [arXiv: 1811.00467] [INSPIRE].

[16] A.V. Belitsky and G.P. Korchemsky, Exact null octagon, JHEP 05 (2020) 070 [arXiv: 1907.13131] [INSPIRE].

[17] A.V. Belitsky and G.P. Korchemsky, Octagon at finite coupling, JHEP 07 (2020) 219 [arXiv: 2003.01121] [INSPIRE].

[18] I. Kostov, V.B. Petkova and D. Serban, Determinant formula for the octagon form factor in $N=4$ supersymmetric Yang-Mills theory, Phys. Rev. Lett. 122 (2019) 231601 [arXiv: 1903.05038] [INSPIRE].

[19] I. Kostov, V.B. Petkova and D. Serban, The octagon as a determinant, JHEP 11 (2019) 178 [arXiv: 1905.11467] [INSPIRE].

[20] T. Fleury and V. Goncalves, Decagon at two loops, JHEP 07 (2020) 030 [arXiv:2004.10867] [INSPIRE].

[21] M. De Leeuw, B. Eden, D. Le Plat, T. Meier and A. Sfondrini, Multi-particle finite-volume effects for hexagon tessellations, JHEP 09 (2020) 039 [arXiv: 1912.12231] [INSPIRE].

[22] T. Bargheer, F. Coronado and P. Vieira, Octagons I: combinatorics and non-planar resummations, JHEP 08 (2019) 162 [arXiv: 1904.00965] [INSPIRE].

[23] T. Bargheer, F. Coronado and P. Vieira, Octagons II: strong coupling, arXiv:1909.04077 [INSPIRE].

[24] T. McLoughlin, R. Pereira and A. Spiering, One-loop non-planar anomalous dimensions in super Yang-Mills theory, JHEP 10 (2020) 124 [arXiv: 2005.14254] [INSPIRE].

[25] T. Bargheer, J. Caetano, T. Fleury, S. Komatsu and P. Vieira, Handling Handles: Nonplanar Integrability in $\mathcal{N}=4$ Supersymmetric Yang-Mills Theory, Phys. Rev. Lett. 121 (2018) 231602 [arXiv: 1711.05326] [inSPIRE].

[26] B. Eden, Y. Jiang, D. le Plat and A. Sfondrini, Colour-dressed hexagon tessellations for correlation functions and non-planar corrections, JHEP 02 (2018) 170 [arXiv:1710.10212] [INSPIRE].

[27] R. Ben-Israel, A.G. Tumanov and A. Sever, Scattering amplitudes - Wilson loops duality for the first non-planar correction, JHEP 08 (2018) 122 [arXiv: 1802.09395] [INSPIRE].

[28] T. Bargheer, J. Caetano, T. Fleury, S. Komatsu and P. Vieira, Handling handles. Part II. Stratification and data analysis, JHEP 11 (2018) 095 [arXiv:1809.09145] [INSPIRE].

[29] Y. Jiang, S. Komatsu and E. Vescovi, Structure constants in $\mathcal{N}=4$ SYM at finite coupling as worldsheet g-function, JHEP 07 (2020) 037 [arXiv: 1906. 07733] [INSPIRE]. 
[30] Y. Jiang, S. Komatsu and E. Vescovi, Exact three-point functions of determinant operators in planar $N=4$ supersymmetric Yang-Mills theory, Phys. Rev. Lett. 123 (2019) 191601 [arXiv: 1907.11242] [INSPIRE].

[31] P. Yang, Y. Jiang, S. Komatsu and J.-B. Wu, D-branes and orbit average, arXiv:2103.16580 [INSPIRE].

[32] Y. Jiang, S. Komatsu, J.-B. Wu and P. Yang, Structure constants in ABJM and integrable bootstrap, to appear.

[33] O. Aharony, O. Bergman, D.L. Jafferis and J. Maldacena, $N=6$ superconformal Chern-Simons-matter theories, M2-branes and their gravity duals, JHEP 10 (2008) 091 [arXiv:0806.1218] [INSPIRE].

[34] J.A. Minahan and K. Zarembo, The Bethe ansatz for superconformal Chern-Simons, JHEP 09 (2008) 040 [arXiv:0806.3951] [INSPIRE].

[35] D. Gaiotto, S. Giombi and X. Yin, Spin chains in $N=6$ superconformal Chern-Simons-matter theory, JHEP 04 (2009) 066 [arXiv: 0806.4589] [INSPIRE].

[36] T. Nishioka and T. Takayanagi, On type IIA Penrose limit and $N=6$ Chern-Simons theories, JHEP 08 (2008) 001 [arXiv:0806.3391] [INSPIRE].

[37] D. Bak and S.-J. Rey, Integrable spin chain in superconformal Chern-Simons theory, JHEP 10 (2008) 053 [arXiv: 0807.2063] [INSPIRE].

[38] A. Cavaglià, D. Fioravanti, N. Gromov and R. Tateo, Quantum spectral curve of the $\mathcal{N}=6$ supersymmetric Chern-Simons theory, Phys. Rev. Lett. 113 (2014) 021601 [arXiv: 1403.1859] [INSPIRE].

[39] D. Bombardelli, A. Cavaglià, D. Fioravanti, N. Gromov and R. Tateo, The full quantum spectral curve for $A d S_{4} / C F T_{3}$, JHEP 09 (2017) 140 [arXiv: 1701. 00473] [INSPIRE].

[40] M. Brockmann, J. De Nardis, B. Wouters and J.S. Caux, A Gaudin-like determinant for overlaps of Néel and XXZ Bethe states, J. Phys. A 47 (2014) 145003 [arXiv:1401.2877].

[41] B. Pozsgay, Overlaps between eigenstates of the XXZ spin-1/2 chain and a class of simple product states, J. Stat. Mech. 06 (2014) P06011 [arXiv:1309.4593].

[42] O. Foda and K. Zarembo, Overlaps of partial Néel states and Bethe states, J. Stat. Mech. 1602 (2016) 023107 [arXiv: 1512.02533] [inSPIRE].

[43] L. Piroli, B. Pozsgay and E. Vernier, What is an integrable quench?, Nucl. Phys. B 925 (2017) 362 [arXiv: 1709.04796] [InSPIRE].

[44] B. Pozsgay, Overlaps with arbitrary two-site states in the XXZ spin chain, J. Stat. Mech. 1805 (2018) 053103 [arXiv: 1801.03838] [INSPIRE].

[45] M. De Leeuw, T. Gombor, C. Kristjansen, G. Linardopoulos and B. Pozsgay, Spin chain overlaps and the twisted yangian, JHEP 01 (2020) 176 [arXiv:1912.09338] [INSPIRE].

[46] B. Pozsgay, L. Piroli and E. Vernier, Integrable matrix product states from boundary integrability, SciPost Phys. 6 (2019) 062 [arXiv: 1812.11094] [INSPIRE].

[47] Y. Jiang and B. Pozsgay, On exact overlaps in integrable spin chains, JHEP 06 (2020) 022 [arXiv:2002.12065] [INSPIRE].

[48] H.-H. Chen, Exact overlaps in the Lieb-Liniger model from coordinate Bethe ansatz, Phys. Lett. B 808 (2020) 135631 [arXiv:2003.02711] [INSPIRE]. 
[49] M. de Leeuw, C. Kristjansen and K. Zarembo, One-point functions in defect CFT and integrability, JHEP 08 (2015) 098 [arXiv:1506.06958] [INSPIRE].

[50] S. Komatsu and Y. Wang, Non-perturbative defect one-point functions in planar $\mathcal{N}=4$ super-Yang-Mills, Nucl. Phys. B 958 (2020) 115120 [arXiv: 2004.09514] [InSPIRE].

[51] I. Buhl-Mortensen, M. de Leeuw, C. Kristjansen and K. Zarembo, One-point functions in AdS/dCFT from matrix product states, JHEP 02 (2016) 052 [arXiv: 1512.02532] [INSPIRE].

[52] M. de Leeuw, C. Kristjansen and S. Mori, AdS/dCFT one-point functions of the SU(3) sector, Phys. Lett. B 763 (2016) 197 [arXiv:1607.03123] [INSPIRE].

[53] I. Buhl-Mortensen, M. de Leeuw, A.C. Ipsen, C. Kristjansen and M. Wilhelm, Asymptotic one-point functions in gauge-string duality with defects, Phys. Rev. Lett. 119 (2017) 261604 [arXiv: 1704.07386] [INSPIRE].

[54] M. De Leeuw, C. Kristjansen and G. Linardopoulos, Scalar one-point functions and matrix product states of AdS/dCFT, Phys. Lett. B $\mathbf{7 8 1}$ (2018) 238 [arXiv:1802.01598] [InSPIRE].

[55] T. Gombor and Z. Bajnok, Boundary states, overlaps, nesting and bootstrapping AdS/dCFT, JHEP 10 (2020) 123 [arXiv:2004.11329] [inSPIRE].

[56] T. Gombor and Z. Bajnok, Boundary state bootstrap and asymptotic overlaps in AdS/dCFT, JHEP 03 (2021) 222 [arXiv:2006.16151] [INSPIRE].

[57] C. Kristjansen, D. Müller and K. Zarembo, Integrable boundary states in D3-D5 dCFT: beyond scalars, JHEP 08 (2020) 103 [arXiv:2005.01392] [INSPIRE].

[58] C. Kristjansen, D. Müller and K. Zarembo, Overlaps and fermionic dualities for integrable super spin chains, JHEP 03 (2021) 100 [arXiv: 2011.12192] [INSPIRE].

[59] G. Chen, R. de Mello Koch, M. Kim and H.J.R. Van Zyl, Absorption of closed strings by giant gravitons, JHEP 10 (2019) 133 [arXiv:1908.03553] [INSPIRE].

[60] D. Berenstein, D.H. Correa and S.E. Vazquez, A study of open strings ending on giant gravitons, spin chains and integrability, JHEP 09 (2006) 065 [hep-th/0604123] [INSPIRE].

[61] A. Ciavarella, Giant magnons and non-maximal giant gravitons, JHEP 01 (2011) 040 [arXiv: 1011.1440] [INSPIRE].

[62] G. Linardopoulos and K. Zarembo, String integrability of defect CFT and dynamical reflection matrices, JHEP 05 (2021) 203 [arXiv: 2102.12381] [INSPIRE].

[63] A. Dekel and Y. Oz, Integrability of Green-Schwarz sigma models with boundaries, JHEP 08 (2011) 004 [arXiv: 1106.3446] [INSPIRE].

[64] D. Young, ABJ $(M)$ chiral primary three-point function at two-loops, JHEP 07 (2014) 120 [arXiv: 1404.1117] [INSPIRE].

[65] D. Young, An extremal chiral primary three-point function at two-loops in ABJ(M), JHEP 12 (2014) 141 [arXiv:1411.0626] [INSPIRE].

[66] M.S. Bianchi, On three-point functions in ABJM and the latitude Wilson loop, JHEP 10 (2020) 075 [arXiv : 2005. 09522] [INSPIRE].

[67] M. Baggio, J. de Boer and K. Papadodimas, A non-renormalization theorem for chiral primary 3-point functions, JHEP 07 (2012) 137 [arXiv:1203.1036] [INSPIRE].

[68] M. Dedushenko, S.S. Pufu and R. Yacoby, A one-dimensional theory for Higgs branch operators, JHEP 03 (2018) 138 [arXiv:1610.00740] [INSPIRE]. 
[69] M. Dedushenko, Y. Fan, S.S. Pufu and R. Yacoby, Coulomb branch operators and mirror symmetry in three dimensions, JHEP 04 (2018) 037 [arXiv:1712.09384] [INSPIRE].

[70] M. Dedushenko, Y. Fan, S.S. Pufu and R. Yacoby, Coulomb branch quantization and abelianized monopole bubbling, JHEP 10 (2019) 179 [arXiv:1812.08788] [INSPIRE].

[71] M. Mezei, S.S. Pufu and Y. Wang, A 2d/1d holographic duality, arXiv:1703.08749 [INSPIRE].

[72] D. Gaiotto and J. Abajian, Twisted M2 brane holography and sphere correlation functions, arXiv: 2004.13810 [INSPIRE].

[73] S.M. Chester, S.S. Pufu and X. Yin, The M-theory S-matrix From ABJM: beyond 11D supergravity, JHEP 08 (2018) 115 [arXiv:1804.00949] [INSPIRE].

[74] D.J. Binder, S.M. Chester and S.S. Pufu, $A d S_{4} / C F T_{3}$ from weak to strong string coupling, JHEP 01 (2020) 034 [arXiv: 1906.07195] [inSPIRE].

[75] S.M. Chester, R.R. Kalloor and A. Sharon, $3 d \mathcal{N}=4$ OPE coefficients from Fermi gas, JHEP 07 (2020) 041 [arXiv:2004.13603] [INSPIRE].

[76] S. Komatsu and Y. Wang, to appear.

[77] G. Chen, R. De Mello Koch, M. Kim and H.J.R. Van Zyl, Structure constants of heavy operators in ABJM and ABJ theory, Phys. Rev. D 100 (2019) 086019 [arXiv:1909.03215] [INSPIRE].

[78] M. Benna, I. Klebanov, T. Klose and M. Smedback, Superconformal Chern-Simons theories and $\mathrm{AdS}_{4} / \mathrm{CFT}_{3}$ correspondence, JHEP 09 (2008) 072 [arXiv:0806.1519] [INSPIRE].

[79] T. Klose, Review of AdS/CFT Integrability. Chapter IV.3: $N=6$ Chern-Simons and strings on $A d S_{4} \times C P^{3}$, Lett. Math. Phys. 99 (2012) 401 [arXiv:1012.3999] [INSPIRE].

[80] J. Escobedo, N. Gromov, A. Sever and P. Vieira, Tailoring three-point functions and integrability, JHEP 09 (2011) 028 [arXiv:1012.2475] [INSPIRE].

[81] T.K. Dey, Exact large R-charge correlators in ABJM theory, JHEP 08 (2011) 066 [arXiv: 1105.0218] [INSPIRE].

[82] S. Chakrabortty and T.K. Dey, Correlators of giant gravitons from dual ABJ(M) theory, JHEP 03 (2012) 062 [arXiv:1112.6299] [InSPIRE].

[83] D. Giovannoni, J. Murugan and A. Prinsloo, The giant graviton on $A d S_{4} \times C P^{3}$-another step towards the emergence of geometry, JHEP 12 (2011) 003 [arXiv:1108.3084] [INSPIRE].

[84] S. Hirano, C. Kristjansen and D. Young, Giant gravitons on $A d S_{4} \times \mathbb{C} P^{3}$ and their holographic three-point functions, JHEP 07 (2012) 006 [arXiv:1205.1959] [INSPIRE].

[85] S. Corley, A. Jevicki and S. Ramgoolam, Exact correlators of giant gravitons from dual $N=4$ SYM theory, Adv. Theor. Math. Phys. 5 (2002) 809 [hep-th/0111222] [INSPIRE].

[86] P. Caputa and B.A.E. Mohammed, From Schurs to giants in ABJ(M), JHEP 01 (2013) 055 [arXiv: 1210.7705] [INSPIRE].

[87] D. Berenstein and D. Trancanelli, Three-dimensional $N=6$ SCFT's and their membrane dynamics, Phys. Rev. D 78 (2008) 106009 [arXiv:0808.2503] [InSPIRE].

[88] T. Nishioka and T. Takayanagi, Fuzzy ring from M2-brane giant torus, JHEP 10 (2008) 082 [arXiv:0808.2691] [INSPIRE]. 
[89] A. Hamilton, J. Murugan, A. Prinsloo and M. Strydom, A note on dual giant gravitons in $A d S_{4} \times C P^{3}, J H E P 04$ (2009) 132 [arXiv:0901.0009] [INSPIRE].

[90] J. Murugan and A. Prinsloo, ABJM dibaryon spectroscopy, JHEP 05 (2011) 129 [arXiv:1103.1163] [INSPIRE].

[91] N. Gutierrez, Y. Lozano and D. Rodriguez-Gomez, Charged particle-like branes in ABJM, JHEP 09 (2010) 101 [arXiv:1004.2826] [InSPIRE].

[92] Y. Lozano, M. Picos, K. Sfetsos and K. Siampos, ABJM baryon stability and Myers effect, JHEP 07 (2011) 032 [arXiv:1105.0939] [InSPIRE].

[93] M. Herrero, Y. Lozano and M. Picos, Dielectric 5-branes and giant gravitons in ABJM, JHEP 08 (2011) 132 [arXiv:1107.5475] [INSPIRE].

[94] Y. Lozano, J. Murugan and A. Prinsloo, A giant graviton genealogy, JHEP 08 (2013) 109 [arXiv: 1305.6932] [INSPIRE].

[95] P. Liendo, C. Meneghelli and V. Mitev, On correlation functions of BPS operators in 3d $\mathcal{N}=6$ superconformal theories, Commun. Math. Phys. 350 (2017) 387 [arXiv:1512.06072] [INSPIRE].

[96] F. Bastianelli and R. Zucchini, Three point functions of chiral primary operators in $d=3$, $N=8$ and $d=6, N=(2,0)$ SCFT at large $N$, Phys. Lett. $B 467$ (1999) 61 [hep-th/9907047] [INSPIRE].

[97] N. Gromov and G. Sizov, Exact slope and interpolating functions in $N=6$ supersymmetric Chern-Simons theory, Phys. Rev. Lett. 113 (2014) 121601 [arXiv:1403.1894] [INSPIRE].

[98] N. Drukker and J. Plefka, Superprotected n-point correlation functions of local operators in $N=4$ super Yang-Mills, JHEP 04 (2009) 052 [arXiv:0901.3653] [INSPIRE].

[99] N. Gorini, L. Griguolo, L. Guerrini, S. Penati, D. Seminara and P. Soresina, The topological line of $A B J(M)$ theory, JHEP 06 (2021) 091 [arXiv: 2012.11613] [INSPIRE].

[100] O. Ohlsson Sax, R. Pereira and A. Sfondrini, unpublished.

[101] R. Pereira, Correlation functions in integrable theories: from weak to strong coupling, Ph.D. thesis, Uppsala University, Uppsala, Sweden (2017).

[102] Y. Kazama, S. Komatsu and T. Nishimura, Novel construction and the monodromy relation for three-point functions at weak coupling, JHEP 01 (2015) 095 [Erratum ibid. 08 (2015) 145] [arXiv: 1410.8533] [INSPIRE].

[103] E. Vescovi, Four-point function of determinant operators in $\mathcal{N}=4$ SYM, Phys. Rev. D 103 (2021) 106001 [arXiv:2101.05117] [INSPIRE].

[104] S. Ghoshal and A.B. Zamolodchikov, Boundary S matrix and boundary state in two-dimensional integrable quantum field theory, Int. J. Mod. Phys. A 9 (1994) 3841 [Erratum ibid. 9 (1994) 4353] [hep-th/9306002] [INSPIRE].

[105] N. Gromov and P. Vieira, Tailoring three-point functions and integrability. Part IV. Theta-morphism, JHEP 04 (2014) 068 [arXiv: 1205.5288] [INSPIRE].

[106] A. A. Vladimirov, Proof of the invariance of the bethe-ansatz solutions under complex conjugation, Theor. Math. Phys. 66 (1986) 102.

[107] J. Caetano and S. Komatsu, Functional equations and separation of variables for exact g-function, JHEP 09 (2020) 180 [arXiv: 2004.05071] [INSPIRE]. 
[108] A. Cavaglià, N. Gromov and F. Levkovich-Maslyuk, Separation of variables in AdS/CFT: functional approach for the fishnet CFT, JHEP 06 (2021) 131 [arXiv:2103.15800] [INSPIRE].

[109] T. Gombor and B. Pozsgay, On factorized overlaps: algebraic Bethe Ansatz, twists, and separation of variables, Nucl. Phys. B 967 (2021) 115390 [arXiv:2101.10354] [InSPIRE].

[110] A. Bissi, C. Kristjansen, A. Martirosyan and M. Orselli, On three-point functions in the $A d S_{4} / C F T_{3}$ correspondence, JHEP 01 (2013) 137 [arXiv:1211.1359] [INSPIRE].

[111] R. de Mello Koch and H.J.R. van Zyl, Inelastic magnon scattering, Phys. Lett. B 768 (2017) 187 [arXiv: 1603.06414] [INSPIRE].

[112] R. de Mello Koch, M. Kim and H.J.R. Zyl, Integrable subsectors from holography, JHEP 05 (2018) 198 [arXiv: 1802.01367] [InSPIRE].

[113] H.-H. Chen, H. Ouyang and J.-B. Wu, Open spin chains from determinant like operators in ABJM theory, Phys. Rev. D 98 (2018) 106012 [arXiv:1809.09941] [InSPIRE].

[114] N. Bai, H.-H. Chen, H. Ouyang and J.-B. Wu, Two-loop integrability of ABJM open spin chain from giant graviton, JHEP 03 (2019) 193 [arXiv: 1901.03949] [INSPIRE].

[115] H.-H. Chen, Asymptotic Bethe ansatz of ABJM open spin chain from giant graviton, JHEP 08 (2019) 109 [arXiv: 1906.09886] [INSPIRE].

[116] N. Gromov and P. Vieira, Complete 1-loop test of AdS/CFT, JHEP 04 (2008) 046 [arXiv: 0709.3487] [INSPIRE].

[117] C. Marboe and D. Volin, Fast analytic solver of rational Bethe equations, J. Phys. A 50 (2017) 204002 [arXiv: 1608.06504] [InSPIRE].

[118] P. Kulish and N. Reshetikhin, Diagonalization of $\mathrm{GL}(N)$ invariant transfer matrices and quantum $N$ wave system (Lee model), J. Phys. A 16 (1983) L591.

[119] N.A. Slavnov, Introduction to the nested algebraic Bethe ansatz, SciPost Phys. Lect. Notes 19 (2020) 1 [arXiv: 1911.12811] [InSPIRE]. 